\title{
A Fully Integrated Distributed Active Transformer Power Amplifier with Injection Locking
}

\author{
by \\ Nicholas John MacDonell Laughton, B. Sc., B.Eng.
}

A thesis submitted to the Faculty of Graduate and Postdoctoral Affairs in partial fulfillment of the requirements for the degree of Master of Applied Science in Electrical and Computer Engineering

Ottawa-Carleton Institute for Electrical and Computer Engineering Carleton University Ottawa, Ontario

(C) 2017, Nicholas John MacDonell Laughton 


\begin{abstract}
This work explores the design of a novel integrated RF power amplifier in IBM's 0.13 micron RF CMOS process. Through a combination of architectures, the series output transformer coupling of a distributed active transformer (DAT) topology is paired with a gain enhancing injection-locking architecture in an attempt to realize a combination of high output power, gain and efficiency; while minimizing die area and supply voltage. The DAT topology simultaneously provides high quality impedance transformation and power combining in the output network that can be used to overcome the low breakdown and high knee voltages - and lossy on-chip passive components - inherent to silicon processes. While improving the overall maximum output power, the large transistors of the DAT require large amplitude driving signals which limit the gain of the system. The injection-locking technique can be used to reduce the input drive voltage for an amplifier circuit, thereby increasing the gain and, consequently, the power-added efficiency. By employing this novel hybrid architecture, the proposed injection-locking DAT power amplifier achieves a $\mathrm{P}_{1 \mathrm{~dB}}$ of $25.94 \mathrm{dBm}$ with a maximum gain of $14.5 \mathrm{~dB}$ and peak PAE of $22 \%$ from a 1.5 $\mathrm{V}$ dc supply voltage (in simulation). The measurement results show discrepancies from the simulated results provided, and several hypotheses as to the cause of these differences are explored.
\end{abstract}




\section{Acknowledgements}

To my supervisor, Dr. Calvin Plett - thank you for your infinite patience

To my wife, Ashley - thank you for your unwavering support

To my daughter, Sawyer - worthwhile achievements are often the hardest 


\section{Table of Contents}

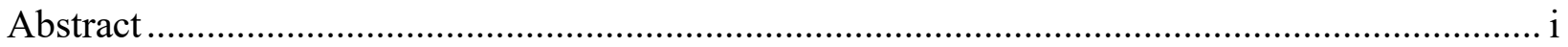

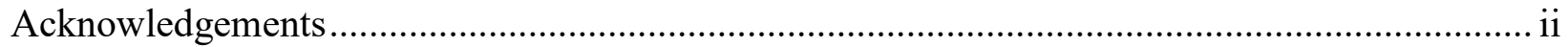

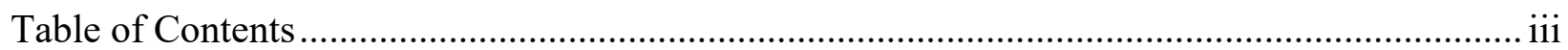

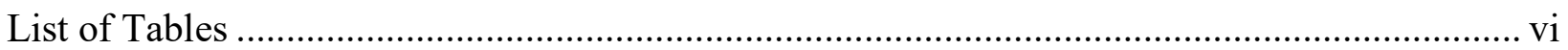

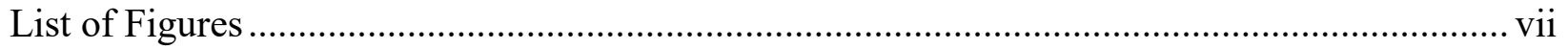

Body

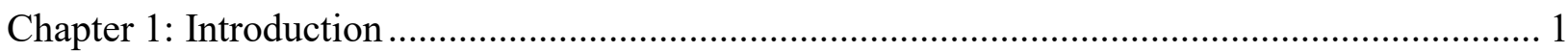

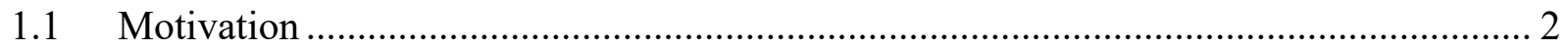

1.2 Objective and Scope

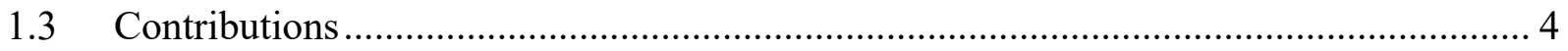

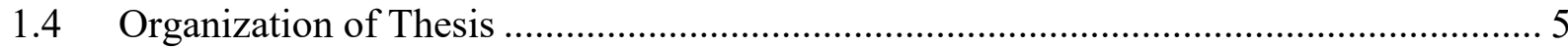

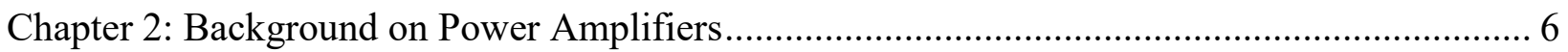

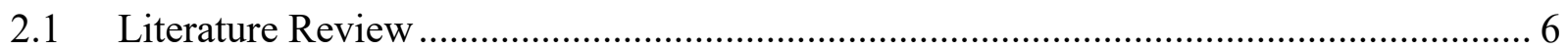

2.2 Fundamentals of Power Amplifier Design ................................................................... 6

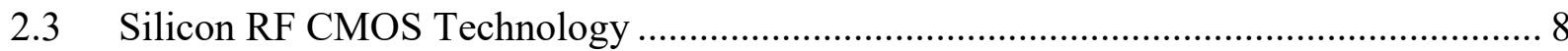

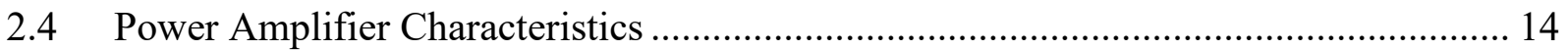

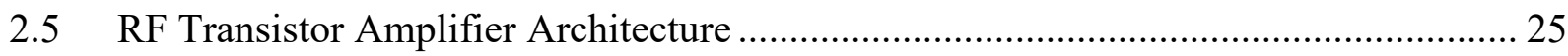

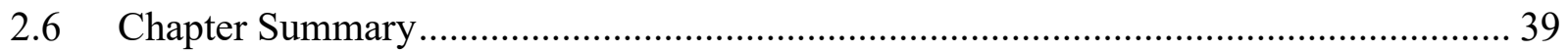

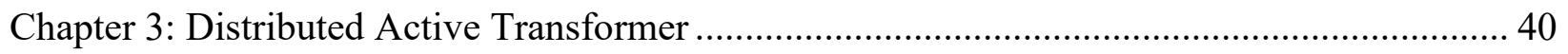




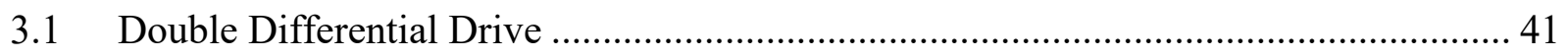

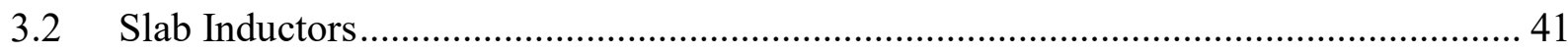

3.3 Circular Geometry and Cross Connected Drain Capacitors............................................ 44

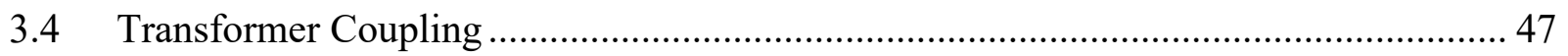

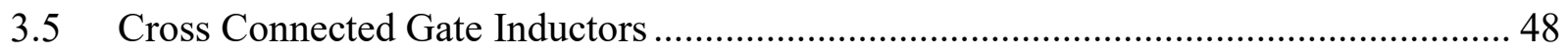

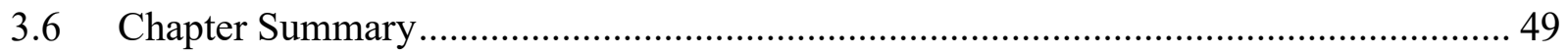

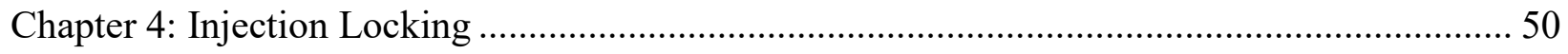

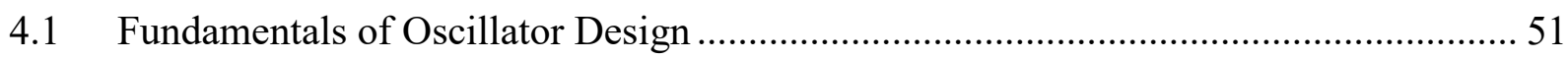

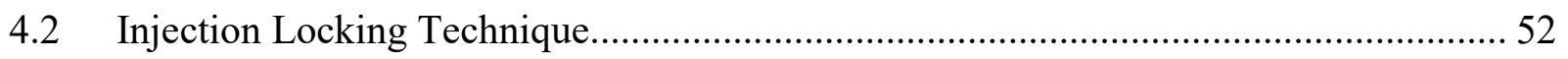

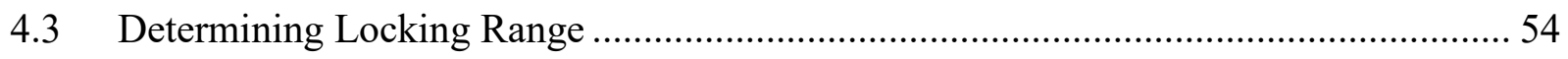

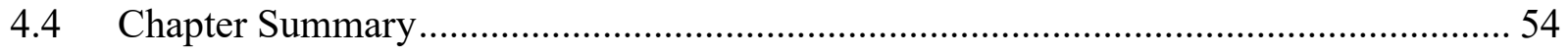

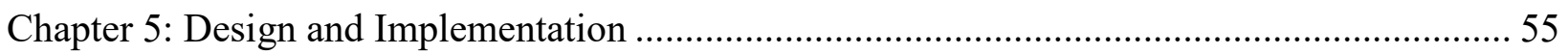

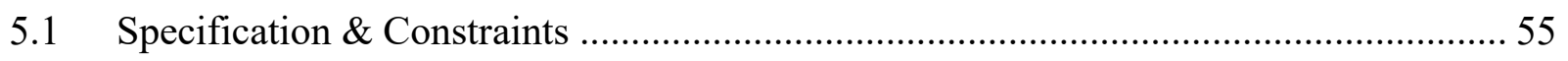

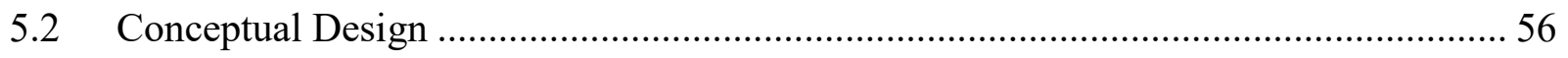

5.3 Distributed Active Transformer Design \& Layout......................................................... 60

5.4 Input Matching Network Design \& Layout …………................................................ 72

5.5 Integration and Top-Level Layout ………………................................................... 77

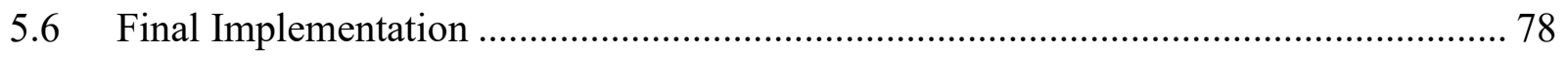

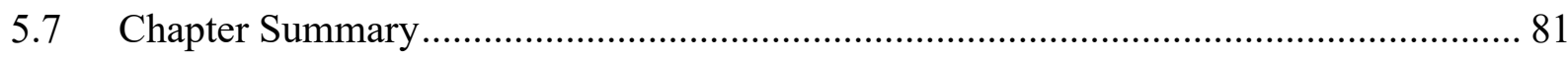

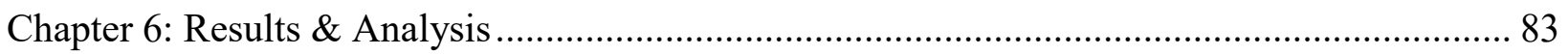




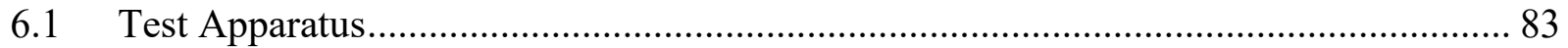

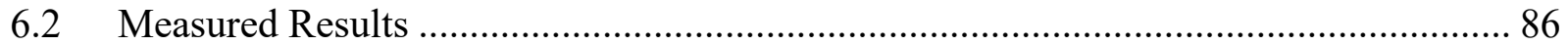

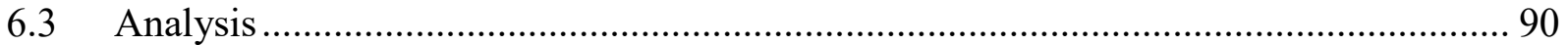

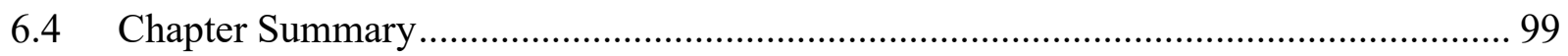

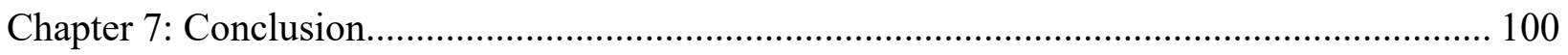

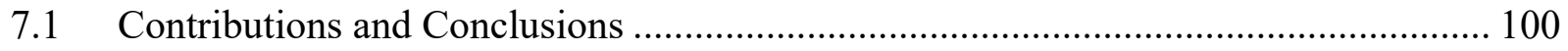

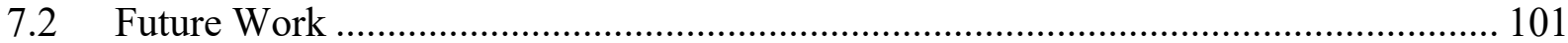

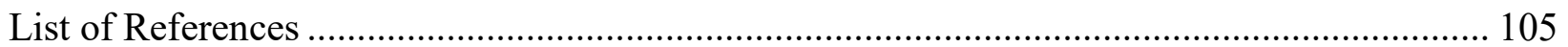




\section{List of Tables}

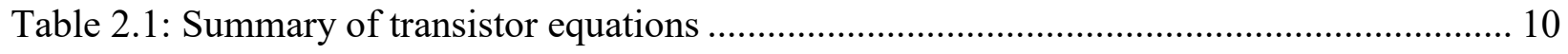

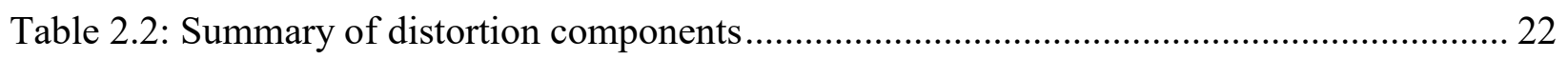

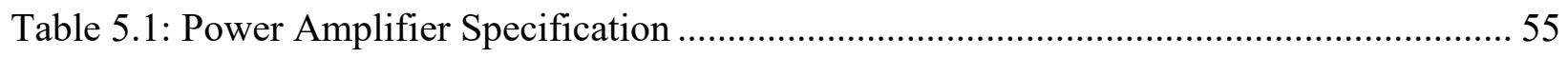

Table 5.2: Active Component Summary - Final Design ............................................................. 79

Table 5.3: Passive Component Summary - Final Design........................................................... 79 


\section{List of Figures}

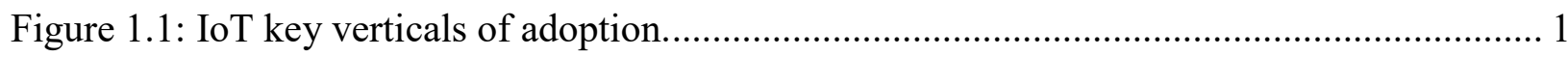

Figure 1.2: Simple transceiver block diagram. ................................................................ 2

Figure 2.1: Generalized two-port transistor amplifier network. ........................................ 7

Figure 2.2: A CMOS nFET with substrate connection................................................. 9

Figure 2.3: CMOS nFET characteristic I-V curves. ...................................................... 10

Figure 2.4: High-frequency small signal $\pi$-model of a CMOS nFET. ................................. 11

Figure 2.5: Generalized two-port transistor amplifier interfaces......................................... 15

Figure 2.6: Ideal and third-order non-linear transfer characteristics. ................................. 20

Figure 2.7: Output spectrum for a third-order nonlinear transfer function............................. 22

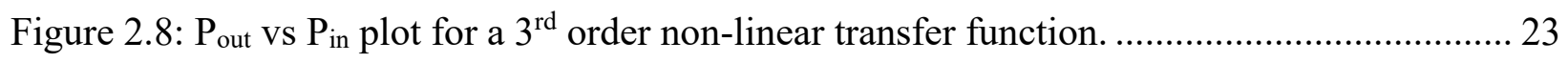

Figure 2.9: Single-stage transistor power amplifier....................................................... 26

Figure 2.10: Class A load line (center) with input voltage (right) and output current (left) and

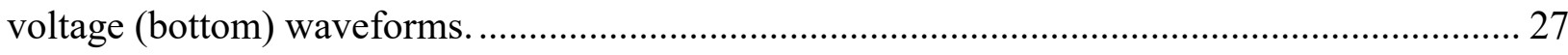

Figure 2.11: Class B load line (center) with input voltage (right) and output current (left) and

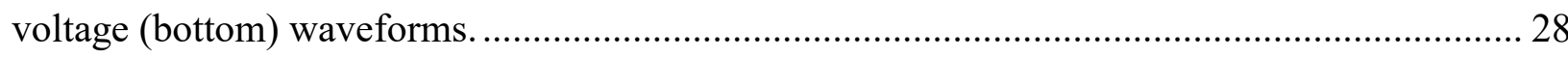

Figure 2.12: Class AB load line (center) with input voltage (right) and output current (left) and

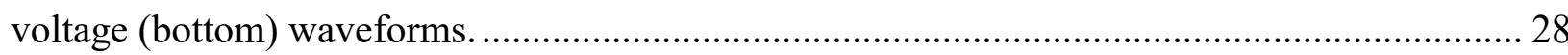

Figure 2.13: Class C load line (center) with input voltage (right) and output current (left) and

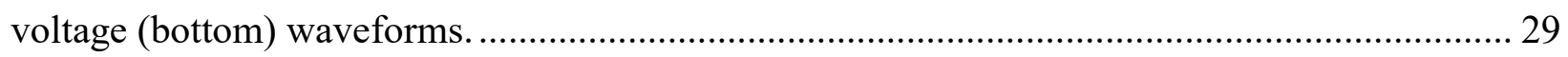

Figure 2.14: Efficiency and normalized output power v. $\alpha$ (degrees). .................................... 30

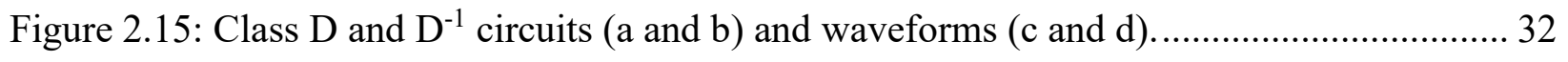

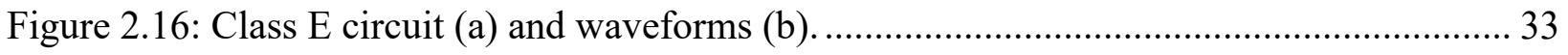


Figure 2.17: Class F amplifier circuit (a) alongside a comparison of class B and F load lines (b)

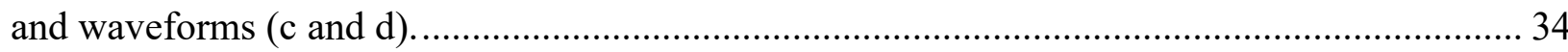

Figure 2.18: Basic lumped-element based impedance matching topologies. .......................... 36

Figure 2.19: Two possible input matching networks for a typical power transistor................... 37

Figure 2.20: Example of input matching with a parallel inductance at the gate input. .............. 37

Figure 2.21: Basic transformer model (a) and equivalent T-model (b) ................................. 38

Figure 2.22: Transformer model showing parasitic capacitances generating lossy L-networks. . 39

Figure 3.1: Push-pull amplifier with a) differential and b) transformer-coupled outputs. .......... 41

Figure 3.2: Smith chart showing inductive and capacitive regions. .................................... 42

Figure 3.3: Lumped-element model of transmission line. .................................................. 42

Figure 3.4: Push-pull amplifier with slab inductor and long interconnects............................ 44

Figure 3.5: Circular geometry with a) conventional and b) adjacent source connections........... 45

Figure 3.6: Circular geometry with adjacent source and drain connections............................ 46

Figure 3.7: Circular geometry with slab inductors and transformer coupling......................... 47

Figure 3.8: Cross connected gate inductor and associated AC ground................................. 48

Figure 4.1: A push-pull amplifier (a), - $g_{m}$ oscillator (b) and injection-locked amplifier (c)....... 50

Figure 4.2: Simplified $-g_{m}$ oscillator (a) with DC (b) and RF (c) equivalent circuits. ............... 51

Figure 4.3: Open-loop model of an oscillator as a feedback control system........................... 52

Figure 4.4: Injection-locking circuit (a) with functional block organization (b) and equivalent

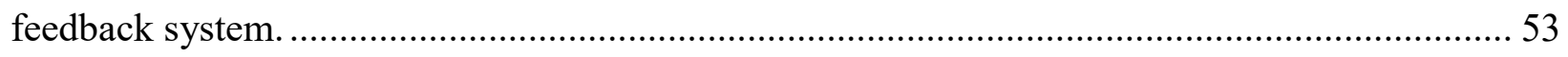

Figure 4.5: Phasor representation of oscillator currents under injection (a) and open loop

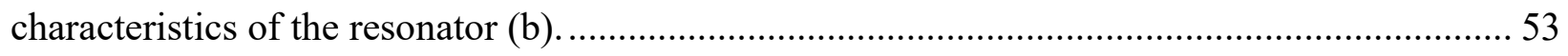

Figure 5.1: Design evolution from conventional DAT to injection-locked DAT..................... 56 
Figure 5.2: Modular topology for circuit design and layout.

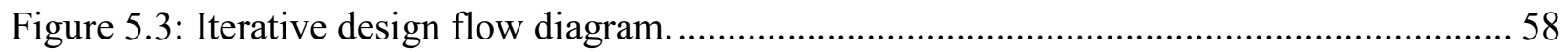

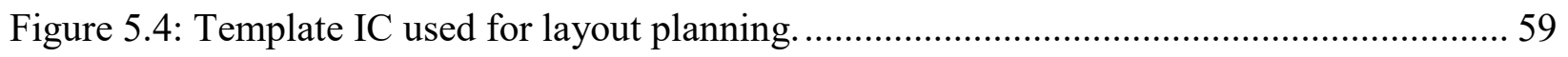

Figure 5.5: Initial floorplan with pad allocation. .............................................................. 59

Figure 5.6: Slab inductance $(\mathrm{pH})$ as a function of length $(\mu \mathrm{m})$ for various widths $(\mu \mathrm{m})$........... 61

Figure 5.7: Quality Factor as a function of length $(\mu \mathrm{m})$ for various widths $(\mu \mathrm{m}) \ldots \ldots \ldots \ldots \ldots \ldots \ldots . . . \ldots 2$

Figure 5.8: Advanced EM simulation of 600 x $50 \mu \mathrm{m}$ slab inductor with center tap................ 63

Figure 5.9: Assumed voltage and current values for output network. .................................... 64

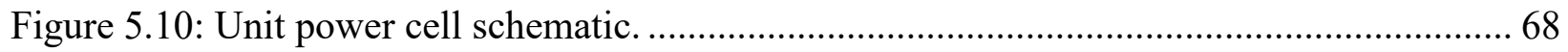

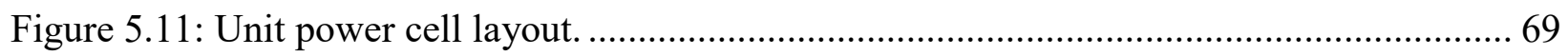

Figure 5.12: The primary and secondary inductances (blue and orange) and quality factors (grey and yellow) for a $600 \mu \mathrm{m}$ coupled line with $5 \mu \mathrm{m}$ spacing and $80 / 20 \mu \mathrm{m}$ widths. ................... 70

Figure 5.13: Output network with slab inductors and magnetic coupling ring. ....................... 71

Figure 5.14: Simulated output power load pull for sizing output tuning capacitance. ............... 72

Figure 5.15: Power divider layout for symmetrical phase shifts. ....................................... 73

Figure 5.16: Power divider insertion loss over frequency. ............................................. 74

Figure 5.17: Power divider phase shift over frequency................................................ 74

Figure 5.18: Integrated balun layout (left) and equivalent electric node map (right)............... 75

Figure 5.19: The primary and secondary inductances (blue and orange) and quality factors (grey

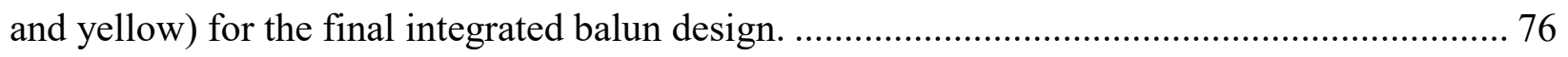

Figure 5.20: Integrated balun schematic model with center tap and tuning capacitors.............. 76

Figure 5.21: Integrated balun layout showing locations of tuning capacitors......................... 77

Figure 5.22: Fully integrated layout with annotations. ................................................ 78 
Figure 5.23: Simulated $\mathrm{S}_{11}$ (blue) and $\mathrm{S}_{21}$ (orange) at over frequency..................................... 80

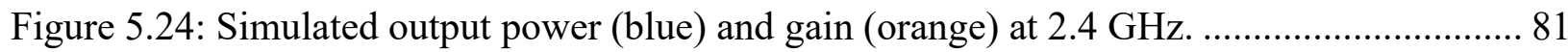

Figure 6.1: Schematic (left) and layout (middle) for the fabricated (right) measurement board.. 84

Figure 6.2: Micrograph of the mounted chip with bond wire connections................................... 84

Figure 6.3: Photograph of the measurement board positioned in the probe station. ..................... 85

Figure 6.4: Block diagrams for the small signal (a) and large signal (b) test benches................. 86

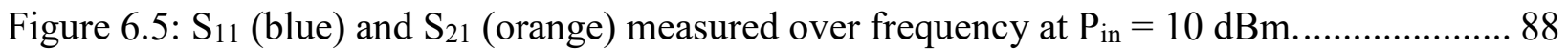

Figure 6.6: Measured output power $(\mathrm{dBm})$ over frequency $(\mathrm{GHz})$ at $\mathrm{P}_{\text {in }}=10 \mathrm{dBm}$.................. 89

Figure 6.7: Measured output power $(\mathrm{dBm})$ over input power $(\mathrm{dBm})$ at $2.4 \mathrm{GHz} . \ldots \ldots \ldots \ldots \ldots \ldots \ldots . . . . . .90$

Figure 6.8: Block diagram for the load pull test bench.......................................................... 92

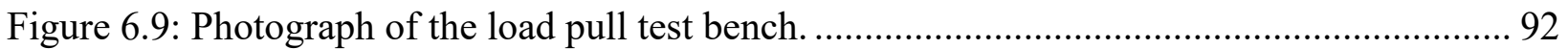

Figure 6.10: Smith chart showing constant gain (blue) and power (red) contour measurements for

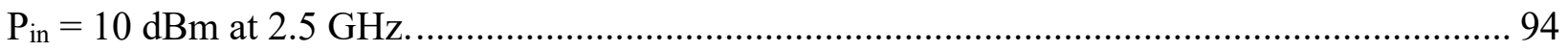

Figure 6.11: $\mathrm{S}_{11}$ (blue) and $\mathrm{S}_{21}$ (orange) measured over frequency with oscillators disabled. ..... 95 Figure 6.12: Output power (blue) and gain (orange) measured over input power with oscillators

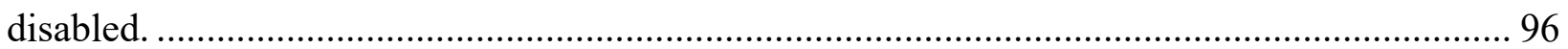

Figure 6.13: Stability factor measured over frequency with oscillators disabled......................... 97

Figure 7.1: Unit power cell with possible location of tuning capacitors.................................. 103 


\section{Chapter 1: Introduction}

The International Telecommunications Union (ITU) views the Internet of Things (IoT) as "a global infrastructure for the information society, enabling advanced services by interconnecting (physical and virtual) things based on existing and evolving interoperable information and communication technologies (ICT)" [1]. By 2020, it is anticipated that adoption of IoT systems will lead to billions of network connected devices across multiple domains - from consumer items such as smart wearables and smart appliances to commercial applications in manufacturing, infrastructure and transportation [2].

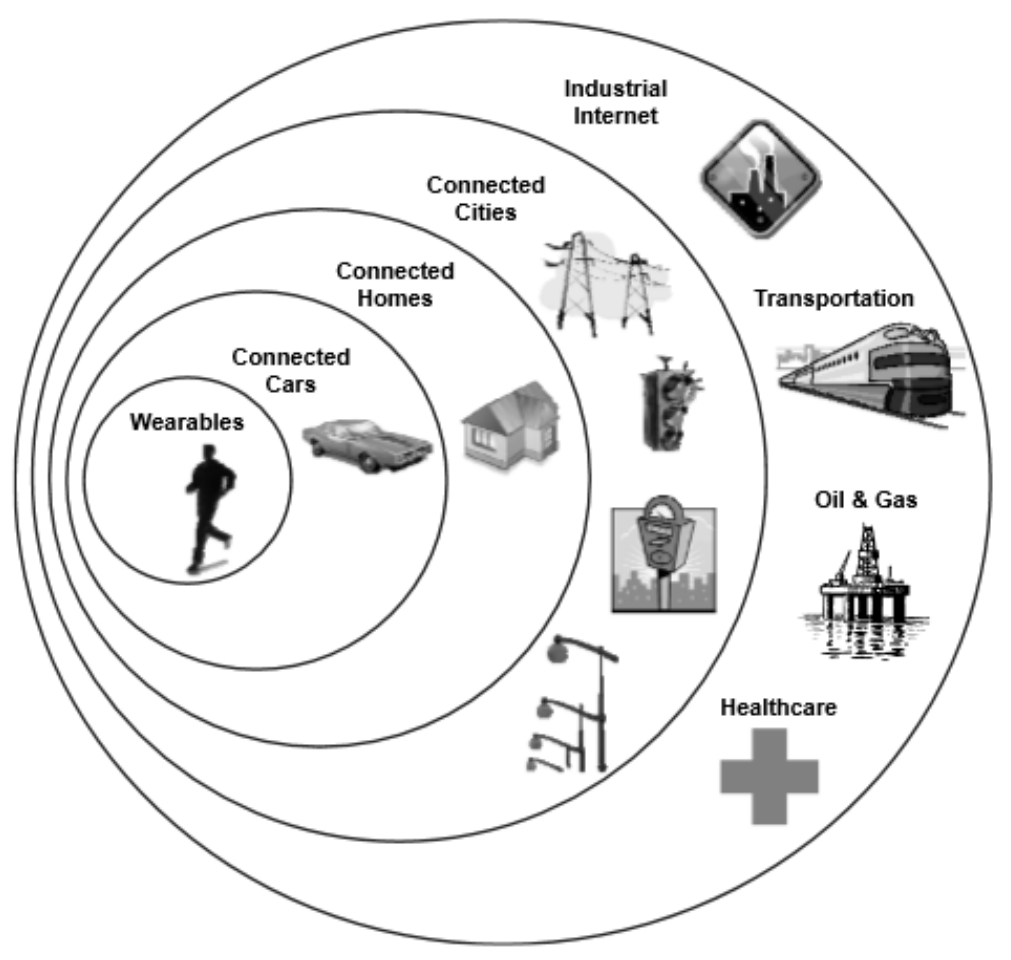

Figure 1.1: IoT key verticals of adoption. ${ }^{1}$

The exponential growth of the cellular market in the early 2000s led to the current stateof-the-art in wireless technology. In a similar fashion, IoT demand for higher data rates with

\footnotetext{
${ }^{1}$ Adapted from [3].
} 
improved quality of service is driving new and challenging technical requirements. In order to meet these requirements while simultaneously closing the business case for IoT device manufacturers, improvements in the size, weight, power and cost of IoT hardware must be realized [2].

\subsection{Motivation}

One approach to reducing size, weight and cost is to combine various circuit blocks into a single integrated circuit - a technique commonly referred to as system-on-a-chip (SOC). The advantages of SOC designs for large scale production are many including higher repeatability and reliability [4]. These, combined with simplified device integration, lead to lower costs and improved margins for manufacturers.

\subsubsection{The Fully Integrated Transceiver}

The fully integrated transceiver is a SOC approach that consolidates the transmitter and receiver functions onto a single chip. Figure 1.2 below shows a generalized transceiver architecture highlighting the baseband and radio-frequency (RF) system blocks.

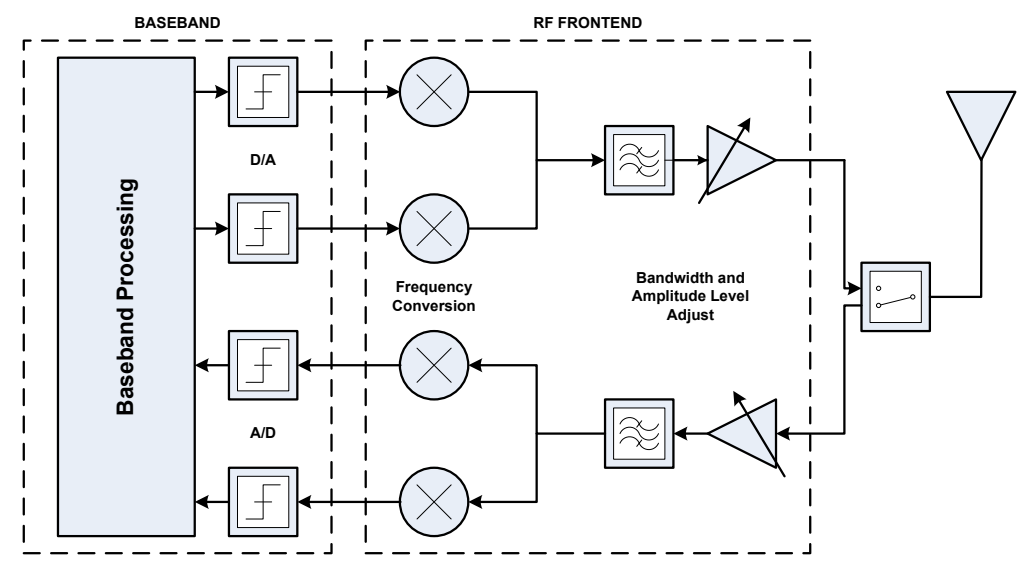

Figure 1.2: Simple transceiver block diagram. ${ }^{2}$

\footnotetext{
${ }^{2}$ Adapted from [10:5].
} 
Although there are clear benefits to high levels of circuit integration, modern transceivers typically maintain a hybrid solution between the baseband and RF circuitry [4]. Baseband circuits are implemented in low-cost silicon complementary metal-oxide-semiconductor (CMOS) processes that are ill-suited to RF circuit design. Instead, RF components are typically implemented in III-V semiconductor processes where the underlying semi-conductor properties are more favourable to achieving high performance. In order to achieve a fully integrated solution, the implementation of an integrated RF front-end in silicon is required and circuit architectures have been developed with a view to overcoming the specific limitations introduced by siliconbased processes.

\subsubsection{Integrated Power Amplifiers - Challenges in Silicon}

RF power amplifiers are critical elements in the transmit chain and - with a few minor exceptions - are typically larger and consume more power than all other elements in the RF front end. They are, therefore, a natural target for integration and much research has been conducted in this area $[4-9 ; 19]$. It has been mentioned that silicon-based processes are ill-suited to RF circuit design and this is especially true of the RF power amplifier. In particular, silicon processes suffer from physical limitations that impose severe challenges on output power, gain and efficiency. First, silicon processes typically have low quality integrated passive components due to the high conductivity substrate (often between 10 and $100 \mathrm{~S} / \mathrm{m}$ ) [11:131]; thereby limiting the efficiency of the input and output impedance matching networks. Secondly, the sub-micron silicon process nodes that enable multi-GHz operation have a combination of low break down voltages and high knee voltages that limit the magnitude of the voltage output swing [4] and consequently the maximum output power. 


\subsection{Objective and Scope}

This work explores the design of an integrated RF power amplifier in IBM's 0.13 micron RF CMOS process. Through a novel combination of architectures, the output transformer coupling of a distributed active transformer (DAT) topology is paired with a gain enhancing injectionlocking architecture in an attempt to realize a combination of high output power, gain and efficiency while minimizing footprint and supply voltage. The DAT topology was developed by Ichiro Aoki in 2002 [12] and simultaneously provides high quality impedance transformation and power combining in the output network that can be used to overcome low breakdown/high knee voltages and low quality factor on-chip passive devices. While improving the overall maximum output power, the large driving transistors of the DAT - requiring large amplitude input signals limit the gain of the system. Injection-locking is a technique for bootstrapping power transistors in order to reduce the input drive voltage for the amplifier circuit, thereby increasing the gain and, consequently, the power-added efficiency (PAE). The DAT topology and injection locking techniques are discussed in further detail in Chapters 3 and 4.

\subsection{Contributions}

The following contributions will be presented in this thesis:

- The conceptual design - a novel integration of the DAT and injection-locking architectures - is successfully designed, implemented and fabricated.

- At the time of submission, this work is believed to be the first attempt at implementing injection-locking in a DAT architecture and represents a 2-3 times improvement in output power to die area ratio when compared with state of the art equivalent PAs

- Simulation results indicating good performance in gain and power are obtained; however, there are discrepancies between these results and the results obtained from measurement. 
- Hypotheses to explain these discrepancies are tested and potential modifications to design methodology and implementation are proposed.

\subsection{Organization of Thesis}

This thesis is organized into seven chapters as follows:

- Chapter 1 introduces the thesis, the motivation for the work and organization of the document.

- Chapters 2-4 explore background literature on power amplifiers and RF CMOS technology, the distributed active transformer architecture and the injection-locking technique.

- Chapter 5 describes the circuit specification, design and implementation.

- Chapter 6 presents and analyzes the measured results, including a discussion of the observed performance deficiencies. Potential root causes for the discrepancies between simulated and realized performance are proposed and evaluated.

- Chapter 7 concludes the thesis and proposes future work on the topic. 


\section{Chapter 2: Background on Power Amplifiers}

This chapter examines the fundamentals of power amplifier design and silicon RF CMOS technology including power amplifier characteristics and transistor amplifier architectures.

\subsection{Literature Review}

Ted Johansson and Jonas Fritzin provide an excellent overview of watt-level CMOS RF power amplifiers in their 2014 review for IEEE Transactions on Microwave Theory and Techniques [4]. In particular, they make the following observations regarding design trends:

- the majority of high power amplifiers used cascode or stacked architectures to enable increasingly high supply voltages while improving gain; and,

- power combining using on-chip transformers is the preferred technique for matching to a $50-\Omega$ load impedance.

As stated in section 1.2, the objective of this work was to unify the power combining architecture of the DAT with the gain enhancement of injection-locking; thereby, enabling a much lower supply voltage than is otherwise required by the more conventional design trends noted above.

\subsection{Fundamentals of Power Amplifier Design}

A useful entry point into the design of any circuit is to establish its purpose or function. For power amplifiers, the purpose is to design an amplifier "to deliver the maximum power output for a given selection of active device" [13:1]. In this context, one can view RF power amplifier design as a sub-modality of the more general field of RF transistor amplifier design - one in which the available design parameters are optimized to meet the above criterion. 
In many cases, RF transistor amplifiers can be practically reduced to a generalized twoport network - described using scattering parameters (S-parameters) - and shown in Figure 2.1 below.

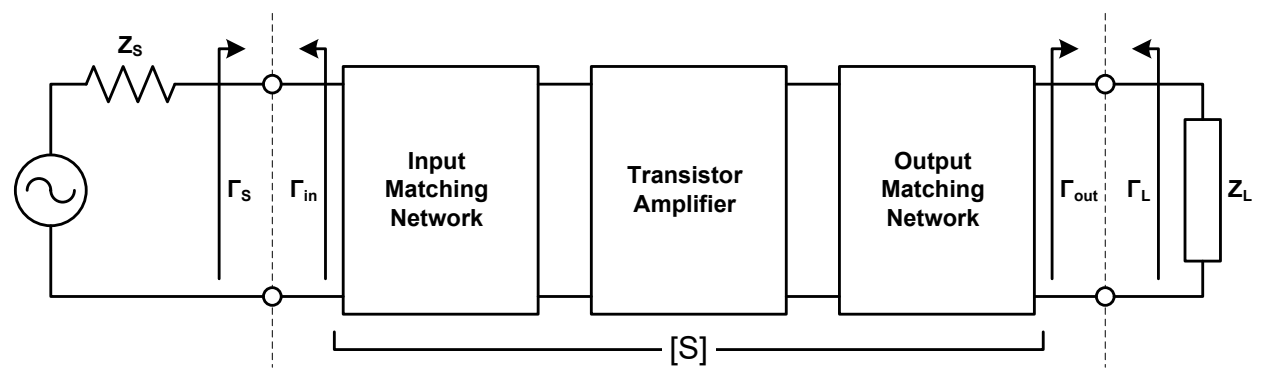

Figure 2.1: Generalized two-port transistor amplifier network. ${ }^{3}$

A two-port active device is connected to a source and load by passive input and output networks, respectively. These networks translate the source and load impedances (typically $50 \Omega$ ) to the optimal input and output impedances required for the desired amplifier function.

The source and load reflection coefficients are given as

$$
\Gamma_{S, L}=\frac{Z_{S, L}-Z_{0}}{Z_{S, L}+Z_{0}}
$$

where $Z_{0}$ is the system reference impedance for $\mathrm{S}$-parameters.

The input and output reflection coefficients can be determined using the above source and load reflection coefficients and are given by

$$
\Gamma_{\text {in }}=S_{11}+\frac{S_{12} S_{21} \Gamma_{L}}{1-S_{22} \Gamma_{L}}
$$

and

$$
\Gamma_{\text {out }}=S_{22}+\frac{S_{12} S_{21} \Gamma_{S}}{1-S_{11} \Gamma_{S}}
$$

\footnotetext{
${ }^{3}$ Adapted from [14:34].
} 
For linear low-noise amplifiers, the input impedance is matched for optimal noise performance and the output impedance is matched for maximum gain and return loss [14:5]. For power amplifiers, the output impedance is matched for maximum power performance and the input impedance is matched for optimal gain and return loss [14:5].

A further consideration for input and output networks is the stability of the amplifier Transistors are not unilateral devices; or, more specifically, the reverse transmission parameter, $S_{12}$, cannot be assumed to be zero [13:3]. As a consequence, reflections at the output can feedback to the input, potentially leading to instability or self-oscillation. Careful design of input and output impedance matching is required to avoid instability. However, this must be balanced against the need to optimize for power and gain performance [13:4].

\subsection{Silicon RF CMOS Technology}

RF CMOS is a low cost, high volume integrated circuit technology that largely came out of university research in the mid-1990s [15]. Over subsequent decades, the technology gained increasing traction on the back of mixed-signal techniques enabled by dramatic reductions in device scale [15]. Although viewed as a key enabler for fully integrated SOC designs, the inherent limitations of lossy passive components and low break down voltages have presented significant barriers to the implementation of power amplifiers in RF CMOS [4].

\subsubsection{The CMOS Transistor and Small Signal Model}

A diagram of a CMOS nFET is shown in Figure 2.2 below. The $\mathrm{nFET}$ consists of a lowresistivity $\mathrm{p}$ - substrate, $\mathrm{n}+$ implants on either side of the channel area for the source and drain, and, a layer of polysilicon over top of a thin layer of oxide for the gate [11:88]. 


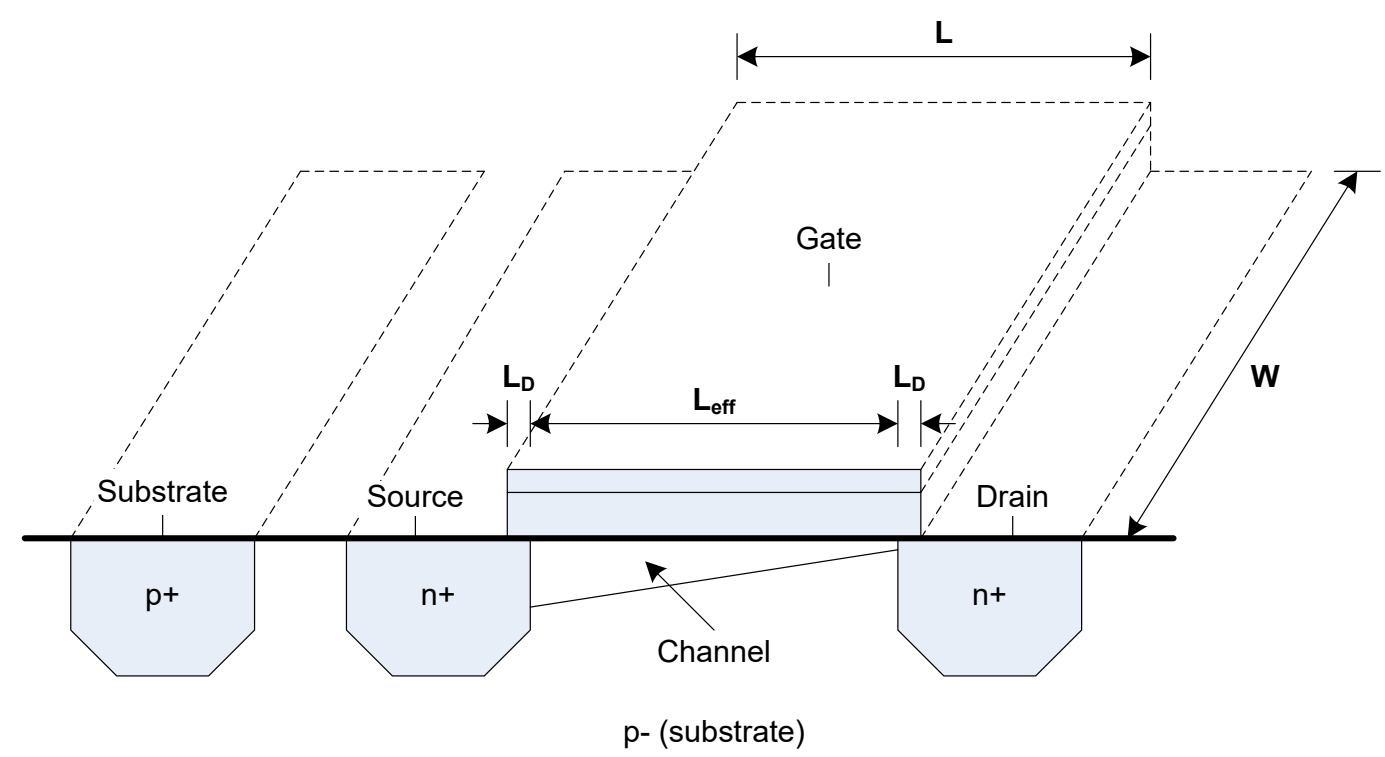

Figure 2.2: A CMOS nFET with substrate connection. ${ }^{4}$

When sufficient voltage is applied at the gate, an n-channel forms in the substrate that allows current to flow between the source and drain in the direction of the applied voltage [11:89]. The minimum gate-source voltage, $V_{g s}$, to establish a channel is referred to as the threshold voltage, $V_{T}$; when $V_{g s}$ is less than $V_{T}$, the transistor is said to be in cutoff. When $V_{g s}$ is greater than $V_{T}$, and the drain-source voltage, $V_{d s}$, is small - the transistor is said to be operating in the triode region. Under these conditions, the channel functions like a resistor and the drain current is predominantly proportional to $V_{d s}$. If $V_{d s}$ gets sufficiently large, the channel begins to taper towards the drain and transistor enters the saturation region in which the drain current is essentially insensitive to variation in $V_{d s}$. The characteristic I-V curves for an nFET are shown in Figure 2.3 below.

\footnotetext{
${ }^{4}$ Adapted from [11:88].
} 


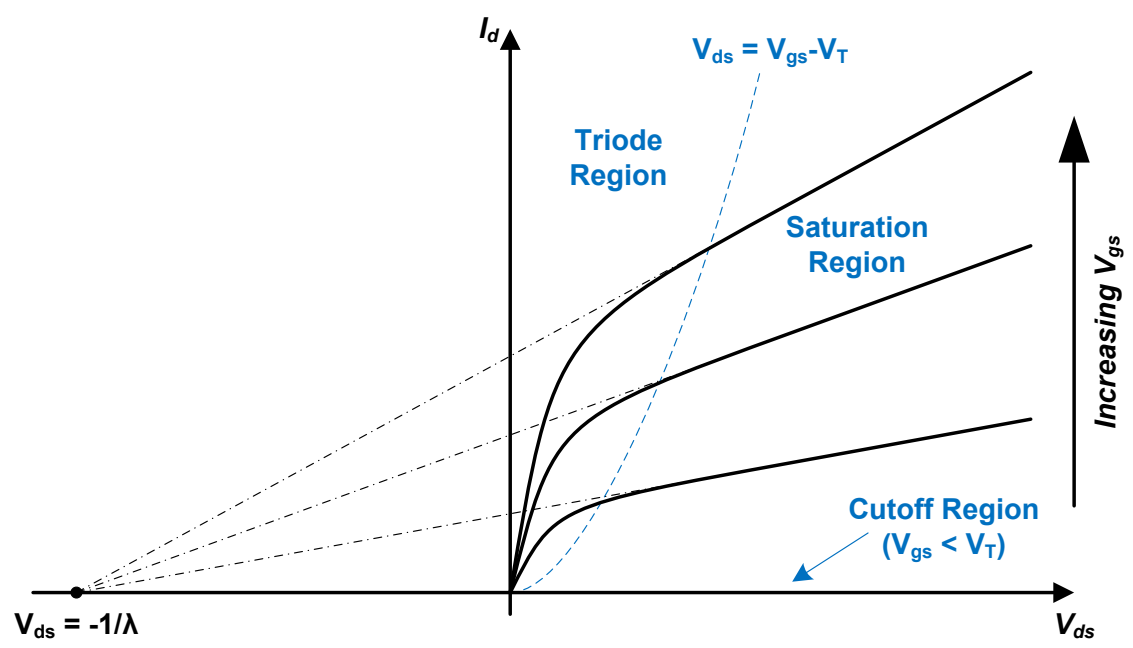

Figure 2.3: CMOS nFET characteristic I-V curves. $^{5}$

Ignoring short channel effects, the operation of the transistor in each region is described using the following basic equations, summarized in Table 2.1 below.

Table 2.1: Summary of transistor equations

\begin{tabular}{ccccc}
\hline Ser & Region & Condition & Equation \\
\hline 1 & Cutoff & $V_{g s} \leq V_{T}$ & $I_{d}=0$ \\
2 & Triode & $V_{g s}>V_{T}, V_{d s} \leq V_{g s}-V_{T}$ & $I_{d}=\mu_{n} C_{o x}\left(\frac{W}{L}\right)\left[\left(V_{g s}-V_{T}\right) V_{d s}-\frac{V_{d s}^{2}}{2}\right]\left(1+\lambda V_{d s}\right)$ & (2.5) \\
3 & Saturation & $V_{g s}>V_{T}, V_{d s}>V_{g s}-V_{T}$ & $I_{d}=\frac{1}{2} \mu_{n} C_{o x}\left(\frac{W}{L}\right)\left(V_{g s}-V_{T}\right)^{2}\left(1+\lambda V_{d s}\right)$
\end{tabular}
where $\mu_{n}$ is the carrier mobility, $C_{o x}$ is the gate capacitance per unit area, $W$ and $L$ are the channel width and length as defined in Figure 2.2, and, $\lambda$ is the output slope factor as defined in Figure 2.3. The voltage that indicates the transition from saturation to triode operation is conventionally referred to as the "knee" voltage (indicating where the I-V curve bends toward the origin).

A more complex formulation of the saturation region equation that includes an additional parameter, $\theta$, for mobility degradation and velocity saturation is given by [11:91]:

\footnotetext{
${ }^{5}$ Adapted from [11:89].
} 


$$
I_{d}=\frac{1}{2} \mu_{n} C_{o x}\left(\frac{W}{L}\right) \frac{\left(V_{g s}-V_{T}\right)^{2}}{1+\theta\left(V_{g s}-V_{T}\right)}\left(1+\lambda V_{d s}\right)
$$

The CMOS transistor can also be represented by a lumped element model for circuit analysis. For high-frequency analysis, this is accomplished by considering the classical lowfrequency small-signal $\pi$-model and adding capacitors to represent the junction and overlap capacitances as shown in Figure 2.4 below.

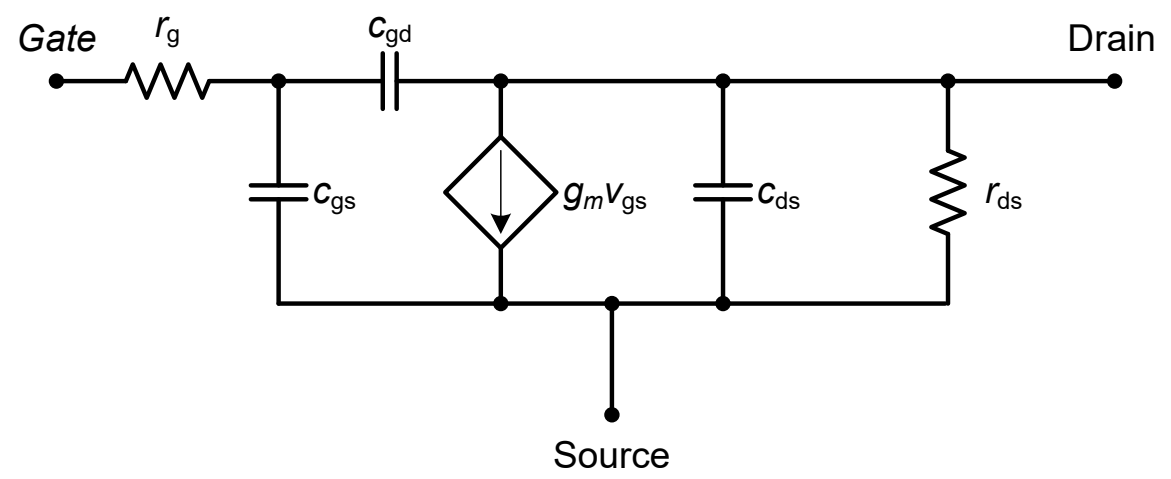

Figure 2.4: High-frequency small signal $\pi$-model of a CMOS nFET. ${ }^{6}$

Several of the model parameters are of particular relevance at RF and will be used to characterize the high frequency performance of the CMOS nFET. The transconductance, $g_{m}$, is determined by taking the derivative of (2.6) with respect to $V_{g s}$ and is given by [11:91]:

$$
g_{m}=\frac{\partial I_{d s}}{\partial V_{g s}}=\mu_{n} C_{o x}\left(\frac{W}{L}\right)\left(V_{g s}-V_{T}\right)\left(1+\lambda V_{d s}\right)
$$

The gate-source capacitance, $C_{g s}$, is the parallel combination of the capacitance generated by the overlap of the gate with the source and some fraction of the gate-channel capacitance, typically given by [11:91]:

$$
C_{g s}=\gamma W L_{e f f} C_{o x}+W L_{D} C_{o v}
$$

\footnotetext{
${ }^{6}$ Adapted from [11:90].
} 
where the parameter $\gamma$ describes the fraction of the channel that is not pinched off under saturation (typically $2 / 3$ for long channel ${ }^{7}$ devices), $C_{o v}$ is the overlap capacitance per unit area, and, $L_{e f f}$ and $L_{D}$ are the effective channel and overlap lengths respectively (as defined in Figure 2.2).

Similar to $C_{g s}$, the gate-drain capacitance, $C_{g d}$, is the parallel combination of the capacitance generated by the overlap of the gate with the drain and some fraction of the gatechannel capacitance. Unlike $C_{g s}$, however, the gate-channel capacitance allocation is reduced to zero under saturation (due to pinchoff) and therefore $C_{g d}$ is given by:

$$
C_{g d}=W L_{D} C_{o v}
$$

The drain-source capacitance, $C_{d s}$, is important for short channel devices however can normally be ignored for long channels where it becomes vanishingly small.

The input gate resistance, $r_{g}$, is determined by the dimensions of the gate connection and whether the gate is contacted from one or both sides [11: 94]. The one sided resistance is given by [11:93]:

$$
r_{g}=\frac{1}{3} \rho_{s} \frac{W}{L}
$$

where $\rho_{S}$ is the gate sheet resistance. The two sided resistance is given by [11:94]:

$$
r_{g}=\frac{1}{12} \rho_{s} \frac{W}{L}
$$

\subsubsection{CMOS Figures of Merit}

There are two key figures of merit that are used to describe the high frequency performance of transistor devices: $f_{t}$, the transit frequency and $f_{\max }$, the maximum frequency.

\footnotetext{
${ }^{7}$ A long channel device has a width and length sufficient large to ignore edge effects.
} 
$f_{t}$ is defined as the frequency at which the unilateral current gain is equal to $0 \mathrm{~dB}[11: 80]$ and can be approximated from the small signal model parameters as follows [11:92]:

$$
f_{t}=\frac{g_{m}}{2 \pi\left(C_{g s}+C_{g d}\right)} \approx \frac{g_{m}}{2 \pi C_{g s}}
$$

where $C_{g s}$ is typically much larger than $C_{g d}$ at saturation.

$f_{\text {max }}$ is defined as the frequency at which the maximum stable power gain is equal to $0 \mathrm{~dB}$ [11:80] and can be approximated from the small signal model parameters as follows [11:92]:

$$
f_{\text {max }}=\sqrt{\frac{f_{t}}{8 \pi \cdot r_{g} C_{g d}}}
$$

From (2.14), we can derive an estimation for the maximum power gain, $G_{\max }$, at a given frequency as follows [11:92]:

$$
G_{\max }(f)=\left(\frac{f_{\max }}{f}\right)^{2}
$$

From these results, it becomes clear that in order to optimize a transistor for maximum power gain at a given frequency, a designer should seek to maximize $f_{\max }$. This in turn creates a trade-off between sizing wide transistors to handle the large currents observed at high power while simultaneously using creative layout techniques to minimize the gate resistance and maximize $f_{\max }$.

\subsubsection{The 0.13 $\mu \mathrm{m}$ RF CMOS Process}

The process technology used in this thesis is the IBM CMOS 8RF process, a high performance $130 \mathrm{~nm}$ CMOS-based technology based on a nonepitaxial p-doped substrate with a substrate conductivity of $1-2 \Omega \mathrm{cm}$. The process stack consists of one poly layer and 8 metal layers:

- three thin copper layers;

- two thick copper layers; and, 
- three RF metal layers consisting of:

$\circ$ a high resistivity aluminum layer;

a low resistivity thick copper layer; and,

○ a low resistivity thick aluminum last metal layer.

The technology also features a variety of devices including thick oxide nFETs with high breakdown voltages and, high quality metal-insulator-metal (MIM) capacitors and spiral inductors.

\subsection{Power Amplifier Characteristics}

In order to optimize transistor amplifiers for power amplification, an understanding of the various performance characteristics is required. For transistor amplifiers, there are five key performance areas that need to be discussed, each addressing different questions about the expected performance of the circuit.

- Power - what output power is delivered to the load?

- Gain - how much input power is required to deliver the output power?

- Efficiency - how much DC power is required to generate the desired output power?

- Linearity - to what extent are the output signal characteristics linear functions of the input signal characteristics?

- Stability - under what input and output conditions will the circuit be well-behaved?

For all five areas, relevant characteristics are evaluated over frequency to determine the functional bandwidth of the power amplifier.

\subsubsection{Power}

There are several useful power characteristics that can be used to describe transistor power amplifiers. They can be divided into input power characteristics and output power characteristics, as discussed below. As a rule, the term available power refers to the power incident to an interface 
and the term delivered power refers to the power transmitted through the interface. In the case of impedance mismatches, some of the available power will be reflected and therefore the delivered power will be reduced. Figure 2.5 below illustrates the input and output interfaces for the twoport amplifier.

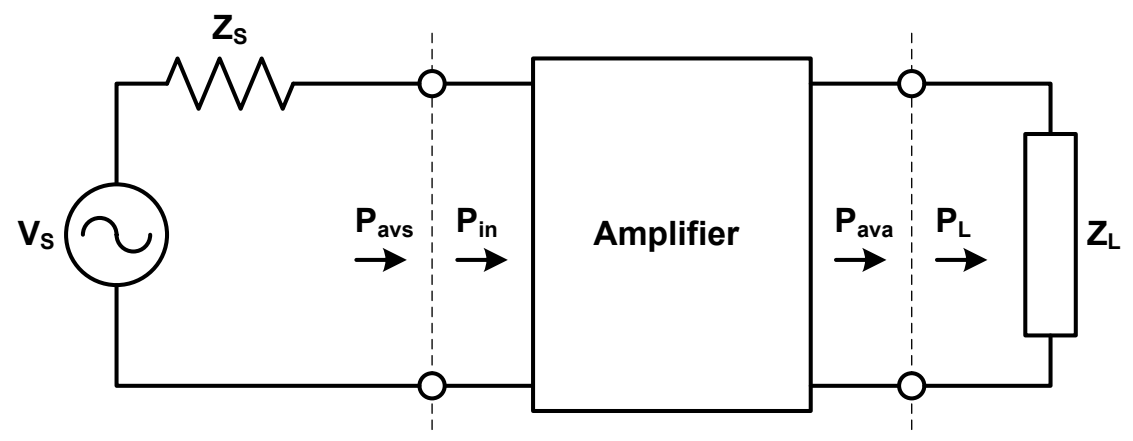

Figure 2.5: Generalized two-port transistor amplifier interfaces.

\subsubsection{Input Power Characteristics}

The input power is defined as the average power delivered to the input network [14:35] and is given by

$$
P_{\text {in }}=\frac{\left|V_{S}\right|^{2}}{8 Z_{0}} \cdot \frac{\left|1-\Gamma_{S}\right|^{2}\left(1-\left|\Gamma_{i n}\right|^{2}\right)}{\left|1-\Gamma_{S} \Gamma_{i n}\right|^{2}}
$$

The maximum power is delivered to the input network when

$$
\Gamma_{\text {in }}=\Gamma_{S}^{*}
$$

Consequently, the available source power is given by

$$
P_{a v s}=\frac{\left|V_{S}\right|^{2}}{8 Z_{0}} \cdot \frac{\left|1-\Gamma_{S}\right|^{2}}{1-\left|\Gamma_{S}\right|^{2}}
$$

and is maximized when the network is matched (i.e. $Z_{S}=Z_{0}$ ).

\subsubsection{Output Power Characteristics}

The output power is defined as the average power delivered to the load [14:35] and is given by 


$$
P_{L}=\frac{\left|V_{S}\right|^{2}}{8 Z_{0}} \cdot \frac{\left|S_{21}\right|^{2}\left(1-\left|\Gamma_{L}\right|^{2}\right)\left|1-\Gamma_{S}\right|^{2}}{\left|1-S_{22} \Gamma_{L}\right|^{2}\left|1-\Gamma_{S} \Gamma_{i n}\right|^{2}}
$$

The maximum power is delivered to the load when

$$
\Gamma_{L}=\Gamma_{\text {out }}^{*}
$$

Consequently, the available amplifier power is given by

$$
P_{\text {ava }}=\frac{\left|V_{S}\right|^{2}}{8 Z_{0}} \cdot \frac{\left|S_{21}\right|^{2}\left(1-\left|\Gamma_{\text {out }}\right|^{2}\right)\left|1-\Gamma_{S}\right|^{2}}{\left|1-S_{22} \Gamma_{\text {out }}^{*}\right|^{2}\left|1-\Gamma_{S} \Gamma_{\text {in }}\right|^{2}}
$$

and is also maximized by matching the output network (i.e. $Z_{L}=Z_{0}$ ).

\subsubsection{Gain}

Power gain is the ratio of output power to input power across the network. There are several definitions of power gain that depend on the output and input power characteristics used to construct the ratio.

\subsubsection{Transducer Power Gain}

The most common definition of power gain is transducer power gain [14:38], defined as the ratio of the output power, $P_{L}$, to the available source power, $P_{a v s}$, and given by:

$$
G_{T}=\frac{P_{L}}{P_{a v s}}=\frac{\left|S_{21}\right|^{2}\left(1-\left|\Gamma_{S}\right|^{2}\right)\left(1-\left|\Gamma_{L}\right|^{2}\right)}{\left|\left(1-S_{11} \Gamma_{S}\right)\left(1-S_{22} \Gamma_{L}\right)-S_{12} S_{21} \Gamma_{S} \Gamma_{L}\right|^{2}}
$$

The transducer power gain is maximized by: a) designing input and output matching networks that simultaneously minimize the source and load reflection coefficients; and, b) sizing the active device to simultaneously maximize the forward transmission parameter while minimizing the reverse. 


\subsubsection{Operating Power Gain}

The operating power gain removes the effect of input mismatches by considering only the source power actually delivered to the input of the amplifier. It is defined as the ratio of the output power, $P_{L}$, to the input power, $P_{\text {in }}$, and is given by [14:38]:

$$
G_{P}=\frac{P_{L}}{P_{i n}}=\frac{\left|S_{21}\right|^{2}\left(1-\left|\Gamma_{L}\right|^{2}\right)}{\left(1-\left|\Gamma_{i n}\right|^{2}\right)\left|1-S_{22} \Gamma_{L}\right|^{2}}
$$

\subsubsection{Available Power Gain}

The available power gain removes the effect of output mismatches by considering only the power available at the load. It is defined as the ratio of the available amplifier power, $P_{a v a}$, to the available source power, $P_{a v s}$, and given by [14:38]:

$$
G_{A}=\frac{P_{a v a}}{P_{a v s}}=\frac{\left|S_{21}\right|^{2}\left(1-\left|\Gamma_{S}\right|^{2}\right)\left(1-\left|\Gamma_{o u t}\right|^{2}\right)}{\left|1-\Gamma_{S} \Gamma_{\text {in }}\right|^{2}\left|1-S_{22} \Gamma_{\text {out }}^{*}\right|^{2}}
$$

\subsubsection{Efficiency}

Efficiency is a measure of how effective the amplifier is at converting supply power to desired RF output power [11:442]. For many applications PA efficiency is critically important. The PA is typically a proportionally large power consumer and therefore reduced efficiency in the PA leads to an overall reduction in battery life (or alternatively increased draw on power supplies). In addition, inefficiency causes heating which can affect the noise performance of the front end receive components (i.e. the LNA) if they are located in close proximity to the PA.

\subsubsection{Drain Efficiency}

The fundamental formulation for single stage amplifiers, referred to as drain efficiency, is defined as the ratio of the output power, $P_{L}$, to the input dc power, $P_{d c}$, and given by [11:442]:

$$
\eta_{\text {drain }}=\frac{P_{L}}{P_{d c}}
$$

where $P_{d c}$ is the product of the supply voltage, $V_{D D}$, and dc component of the drain current, $I_{d}$. 


\subsubsection{Power-added Efficiency}

For amplifiers with low gain, the drain efficiency may be misleading because it does not account for substantial input RF power. Power-added efficiency (PAE) addresses this by considering only the additional RF power generated by the amplifier. It is defined as the ratio of the added RF power to the input DC power and is given by

$$
P A E=\frac{P_{L}-P_{a v s}}{P_{d c}}
$$

Given (2.20), PAE can be alternatively calculated as follows

$$
P A E=\frac{P_{L}-\frac{P_{L}}{G_{T}}}{P_{d c}}=\eta_{\text {drain }} \cdot\left(1-\frac{1}{G_{T}}\right)
$$

From the above, it is clear that in order to maximize PAE, a designer must simultaneously optimize for drain efficiency and gain.

\subsubsection{Linearity}

To this point, for mathematically simplicity, the analysis has considered an ideal active device that can be fully modelled as linear memoryless system. This suggests several assumptions, outlined as follows.

First, it is assumed that the impulse response, $\boldsymbol{H},-$ being linear - obeys the superposition principle and therefore satisfies the following

$$
\begin{array}{cc}
\boldsymbol{H}\left\{x_{1}(t)+x_{2}(t)\right\}=\boldsymbol{H}\left\{x_{1}(t)\right\}+\boldsymbol{H}\left\{x_{2}(t)\right\} & \boldsymbol{H}\left\{\boldsymbol{\alpha} \boldsymbol{x}_{\mathbf{1}}(\boldsymbol{t})\right\}=\boldsymbol{\alpha} \boldsymbol{H}\left\{\boldsymbol{x}_{\mathbf{1}}(\boldsymbol{t})\right\} \\
\text { (additive system) } & \text { (homogeneous system) }
\end{array}
$$

Second, it is assumed that the output at any time $t$ is dependent only on the input at time $t$, with the corollary that a perturbation of the input is instantaneously resolved to the output.

For transistor amplifiers, this implies that the transfer function can be fully described by the following first-degree power series 


$$
v_{o}=a_{1} v_{i}
$$

in which $a_{1}$ is the voltage gain and $v_{i}$ and $v_{o}$ are the input and output small signal voltage waveforms, respectively [13:5]. For small signal variation around the transistor operating point and with passive components whose time constants are much smaller than the rate of change of the input - this model proves to be reasonably accurate. Consequently, since a significant tranche of RF design is conducted under these conditions, the linear memoryless amplifier model forms a normative basis for transistor amplifier analysis.

According to Cripps [13:5], however, the assumptions underpinning this model no longer hold for power amplifiers due to the presence of "strong" and "weak" nonlinear effects that occur at large variations from the operating point: the first caused by the limiting behavior of the transistor [13:6] and, the second caused by reactive passive components introducing phase distortions (also referred to as "memory effects") [13:5].

\subsubsection{Strongly nonlinear effects}

The "ideal" strong nonlinear device can be modelled by taking the first-order power series (here formulated to describe drain current as a function of gate-source voltage) and adding output limits conditional on the magnitude of the input, yielding the following

$$
I_{d}=\left\{\begin{aligned}
0, & V_{g S}<V_{T} \\
g_{1} V_{g s}, & V_{T}<V_{g S}<V_{o c} \\
I_{\max }, & V_{g s} \geq V_{o c}
\end{aligned}\right.
$$

The first limit occurs when the transistor is in cutoff [13:6], as described in section 2.3.1. Under this condition, no drain current is present because the gate-source voltage is insufficient to create a drain-source channel.

The second limit occurs when the drain-source channel is completely open and the maximum drain current is reached. This occurs when the overdrive voltage, $V_{o v}$, is greater than 
$V_{d s}$ and the transistor shifts to the triode region of operation. Under this condition, referred to here as open channel [13:6], the drain-source voltage has a dominant effect on the drain current.

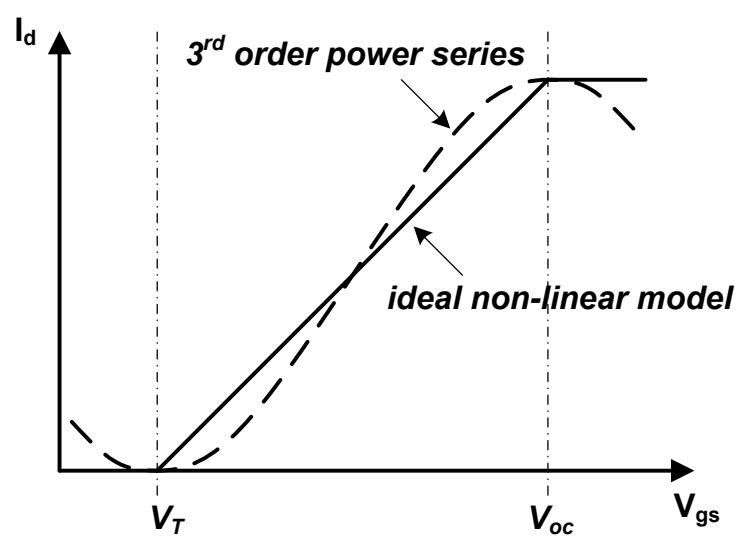

Figure 2.6: Ideal and third-order non-linear transfer characteristics. ${ }^{8}$

The ideal nonlinear model leads to abrupt transitions in transconductance values (Figure 2.6); however, in practice, most FETs exhibit a gradual fading of the transconductance as either condition is approached [13:7]. This property of fading transconductance is considered a thirdorder effect [13:7] and can therefore be effectively modeled using a third-degree power series of the form

$$
I_{d}=g_{0}+g_{1} \cdot V_{g s}+g_{2} \cdot V_{g s}^{2}+g_{3} \cdot V_{g s}^{3}
$$

where $\left\{g_{0} \ldots g_{3}\right\}$ are the model transconductance coefficients.

Having now established that a third order power series is sufficient to model the strongly nonlinear behavior of the transistor, a Taylor series expansion of (2.7) can be used to determine the transconductance coefficients in (2.28). Substituting $V_{o v}$ in place of $V_{g s}-V_{T}$ and allowing the constant $K$ to collect static device parameters, (2.7) can be re-written as

$$
I_{d}=K \frac{V_{o v}{ }^{2}}{1+\theta V_{o v}}\left(1+\lambda V_{d s}\right)
$$

\footnotetext{
${ }^{8}$ Adapted from [8:6].
} 
and the Taylor series expansion is given by

$$
I_{d}\left(v_{g s}\right) \approx I_{d}\left(V_{o v}\right)+\frac{\partial I_{d}\left(V_{o v}\right)}{\partial V_{g s}} v_{g s}+\frac{1}{2 !} \cdot \frac{\partial^{2} I_{d}\left(V_{o v}\right)}{\partial V_{g s}^{2}} v_{g s}^{2}+\frac{1}{3 !} \cdot \frac{\partial^{3} I_{d}\left(V_{o v}\right)}{\partial V_{g s}^{3}} v_{g s}^{3}
$$

where $v_{g s}$ is the small signal excursion from the dc overdrive voltage $V_{o v}$. Collecting like terms between (2.28) and (2.30), and noting that $I_{d}$ is itself the sum of a dc component and an ac small signal component, the small signal drain current can be expressed as

$$
i_{d s} \approx g_{1} \cdot v_{g s}+g_{2} \cdot v_{g s}^{2}+g_{3} \cdot v_{g s}^{3}
$$

where

$$
\begin{gathered}
g_{1}=K \frac{V_{o v}\left(2+\theta V_{o v}\right)}{\left(1+\theta V_{o v}\right)^{2}}\left(1+\lambda V_{d s}\right) \\
g_{2}=K \frac{1}{\left(1+\theta V_{o v}\right)^{3}}\left(1+\lambda V_{d s}\right) \\
g_{3}=-K \frac{\theta}{\left(1+\theta V_{o v}\right)^{4}}\left(1+\lambda V_{d s}\right)
\end{gathered}
$$

From this result, it can be observed that the nonlinear quality for a given transistor geometry is highly dependent on both the dc overdrive voltage and the drain bias voltage.

\subsubsection{Gain Compression and Intermodulation}

A common method for testing the linearity of an amplifier is the two-tone test [10:90]. In this test, a composite input waveform consisting of two equal amplitude sinusoids is applied to the input of the circuit and the output spectrum is observed. The composite input waveform is given by

$$
v_{i n}=A \cdot\left[\cos \left(2 \pi f_{1} t\right)+\cos \left(2 \pi f_{2} t\right)\right]
$$

where $A$ is the voltage amplitude and $f_{1}$ and $f_{2}$ are the different stimulus frequencies. 
As the composite input waveform passes through the amplifier non-linearity, the sinusoids mix and generate harmonic (HD) and intermodulation (IM) products [10:90] as shown in Figure 2.7 .

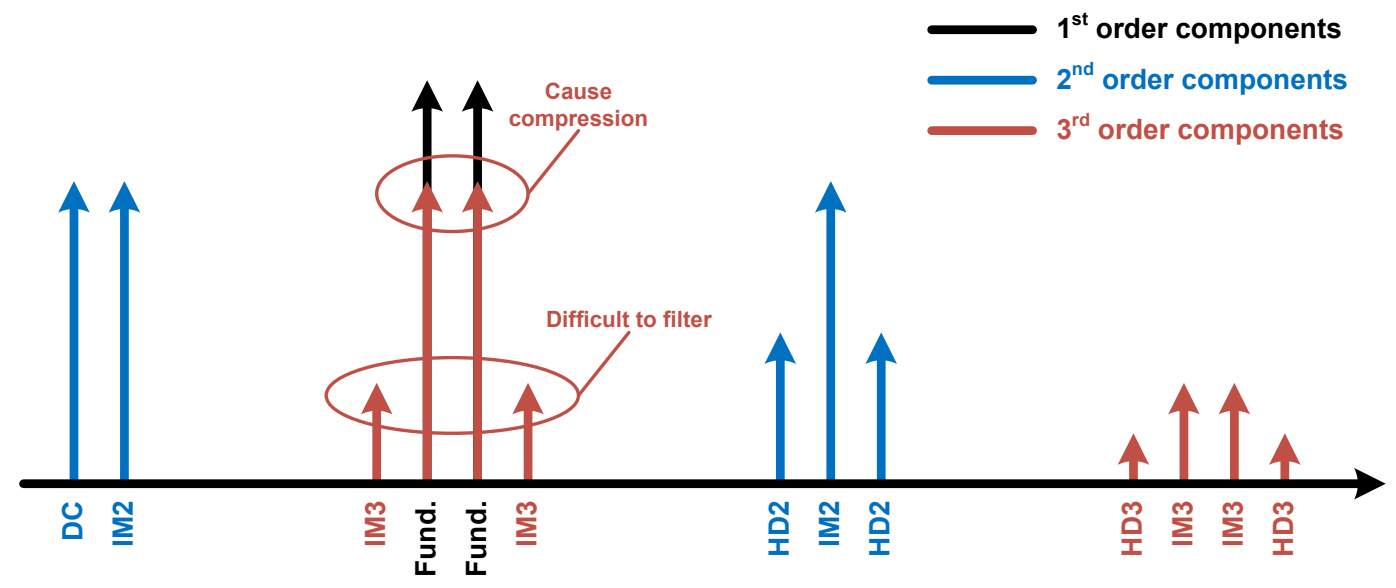

Figure 2.7: Output spectrum for a third-order nonlinear transfer function. ${ }^{9}$

By applying the composite two-tone input (2.35) to $3^{\text {rd }}$ order model from (2.31), equations for the various output current amplitudes of the frequency components can be extracted [10:92] and expressed in terms of the transconductance coefficients (Table 2.2).

Table 2.2: Summary of distortion components ${ }^{10}$

\begin{tabular}{cccc}
\hline Ser & Frequency & Type & Equation \\
\hline \hline 1 & $f_{1}, f_{2}$ & Fundamental & $g_{1} A+\frac{9}{4} g_{3} A^{3}$ \\
2 & $2 f_{1}, 2 f_{2}$ & HD2 & $\frac{1}{2} g_{2} A^{2}$ \\
3 & $f_{2} \pm f_{1}$ & IM2 & $g_{2} A^{2}$ \\
4 & $3 f_{1}, 3 f_{2}$ & HD3 & $\frac{1}{4} g_{3} A^{3}$ \\
5 & $2 f_{2} \pm f_{1}, 2 f_{1} \pm f_{2}$ & IM3 & $\frac{3}{4} g_{3} A^{3}$
\end{tabular}

${ }^{9}$ Adapted from [10:94].

${ }^{10}$ Adapted from [10:92]. 
In general, the primary concern is with the $3^{\text {rd }}$ order products at the fundamental and intermodulation frequencies. The $3^{\text {rd }}$ order fundamental components appear at the desired frequencies and cause gain compression [10:93], reducing the PAE. The $3^{\text {rd }}$ order intermodulation (IM3) products appear adjacent to the fundamental frequencies and are difficult to filter due to their proximity to the desired signals [10:93].

Figure 2.8 shows a plot of the fundamental and intermodulation product output powers as a function of the input power for the $3^{\text {rd }}$ order model. From this plot, several linearity figures of merit are extracted.

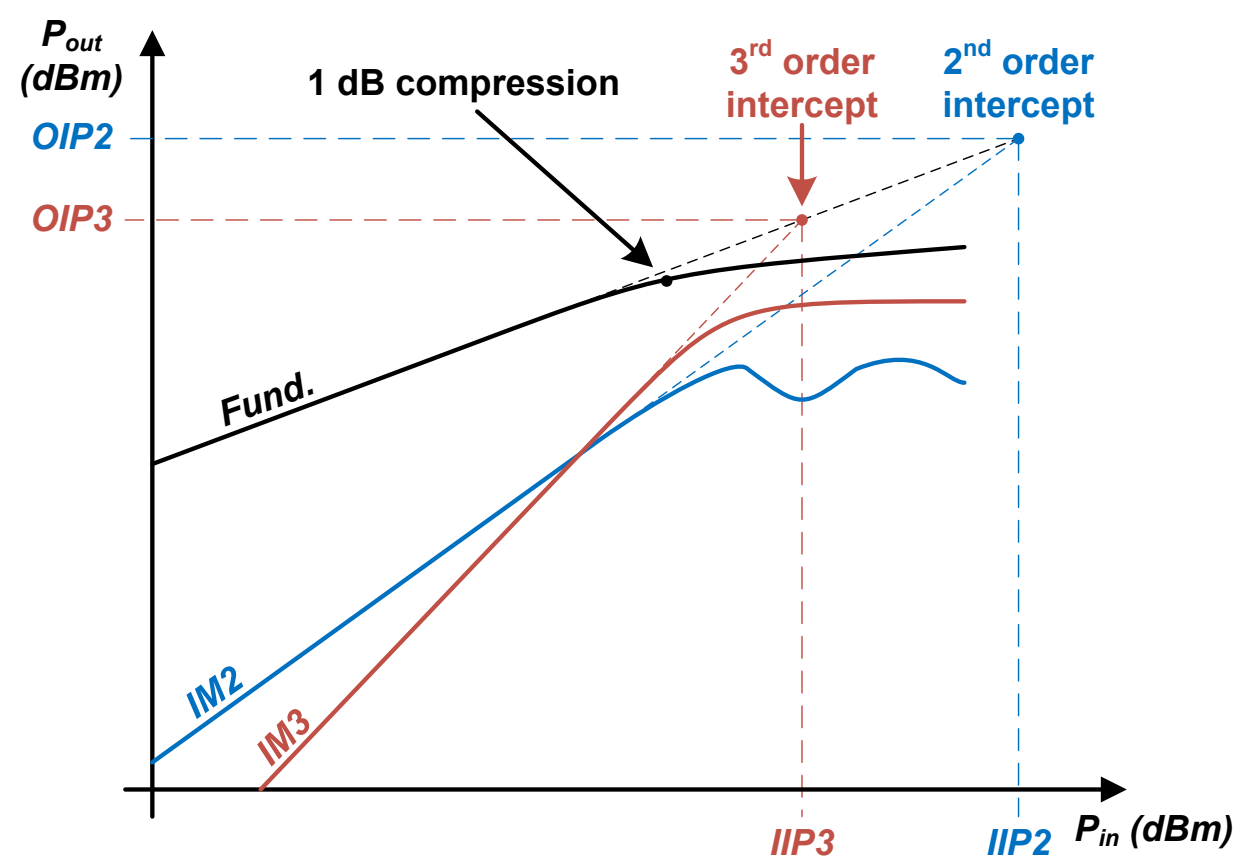

Figure 2.8: $\mathrm{P}_{\text {out }} \mathrm{VS} \mathrm{P}_{\text {in }}$ plot for a $3^{\text {rd }}$ order non-linear transfer function. ${ }^{11}$

As the input power is increased, the fundamental output power increases as a linear function of the input power until the amplifier begins to asymptote towards the saturated output power (due to the limiting effects of the transistors). The point at which the output power is

\footnotetext{
${ }^{11}$ Adapted from [10:94].
} 
reduced by $1 \mathrm{~dB}$ relative to a linear extrapolation of the fundamental curve is known as the $1 \mathrm{~dB}$ compression, or $P_{1 d B}$ (in effect the gain has been reduced by $1 \mathrm{~dB}$ at this point).

The intercept points between the linear extrapolation of the fundamental curve and intermodulation curve are also used to define figures of merit for linearity. These intercepts can be input (IIP) or output (OIP) referred, depending on whether they are specified at the input or output of the amplifier [10:95] and are the theoretical points where the amplitude of the higher order intermodulation products are equal to the fundamental tones. Using the equations from Table 2.2 and equating the linear term from (2.36) equal to (2.40) gives

$$
g_{1} A_{I P 3}=\frac{3}{4} g_{3} A_{I P 3}^{3}
$$

Solving for the amplitude and substituting the expressions for $g_{1}$ and $g_{3}$ gives the following expression for the input-referred IP3 voltage amplitude

$$
A_{I P 3}=2 \sqrt{\frac{V_{o v}\left(2+\theta V_{o v}\right)\left(1+\theta V_{o v}\right)^{2}}{3 \theta}}
$$

From this result, it is observed that increasing the overdrive voltage will improve the linear performance of a transistor - implying that for a given gate bias, transistors with lower threshold voltages will be more linear.

\subsubsection{Weakly nonlinear effects}

While the Taylor expansion is a useful and reasonable approximation of the strongly nonlinear effects that arise from the limiting behaviour of the transistor, the formulation breaks down when memory is introduced into the system [13:5]. Memory arises in transistor amplifiers from several sources - in particular the extrinsic junction capacitances in the active devices and the passive components in the input and output matching and bias networks. Memory effects can be captured by a more robust series expansion known as the Volterra series [13:5] however, in 
practice, this level of accuracy is only required when considering operation past $P_{1 d B}$ and will not be considered in this thesis.

\subsubsection{Stability}

When discussing stability in 2-port networks, the typical starting point is the $k$ parameter, also known Rollett's stability factor [13:3]. In the context of non-zero reverse transmission gain, there may exist passive external circuit configurations that lead to instability; in which unwanted oscillations interfere with circuit functionality. The $k$ parameter is a useful method of checking of unconditional stability, and can be derived from S-parameters as follows

$$
k=\frac{1-\left|S_{11}\right|^{2}-\left|S_{22}\right|^{2}+|\Delta|^{2}}{2 \cdot\left|S_{21}\right| \cdot\left|S_{12}\right|}
$$

where $\Delta$ is the determinant of the 2-port S-parameter matrix [11:214]. When designing RF integrated circuits, the $k$ parameter is typically determined through simulation rather than calculated directly [11:214].

When $k>1$, the circuit is unconditionally stable over all physically realizable passive external circuits. However, Cripps [13:4] notes that devices with $k$ factors significantly greater than unity will have low gain (and therefore reduced efficiency). Therefore, it is favourable to seek designs with $k$ factors only marginally greater than unity and in some cases less than unity (when the presence of known parasitic losses are expected to dampen unwanted oscillations) $[13: 4]$.

\subsection{RF Transistor Amplifier Architecture}

In the introduction to this chapter, a generalized two port amplifier model was reviewed. This section will descend into the two-port amplifier block and examine a single-stage transistor 
power amplifier circuit. The canonical circuit topology for a single stage transistor power amplifier is provided in Figure 2.9 below.

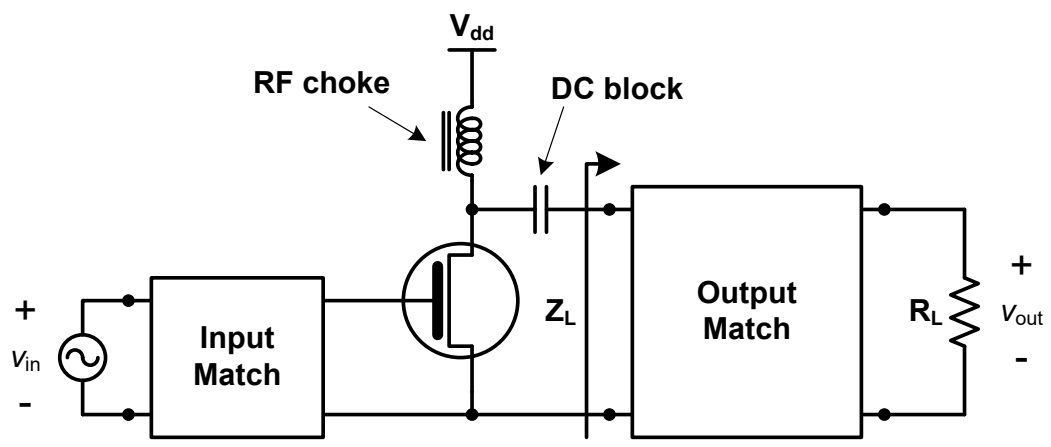

Figure 2.9: Single-stage transistor power amplifier. ${ }^{12}$

The circuit consists of three sub-circuits:

- the active device and bias network;

- the input matching network; and,

- the output matching network.

The following subsections will review the functions of each sub-circuit, providing background on classes of amplifier operation and characteristics of passive networks.

\subsubsection{Active Device and Bias Network}

Transistor power amplifiers are broadly organized according to the operational mode of the active device; "transconductance amplifiers" operate the transistor as a voltage controlled current source whereas "switched mode amplifiers" operate the transistor as a voltage controlled switch. For transconductance amplifiers, active device biasing is the fundamental design consideration and determines the so-called "class" of operation - where the class is determined according to the output current and voltage waveform characteristics [11:444]. Switched mode amplifiers, on the other hand, are all biased to the same switching operation and their class is

\footnotetext{
${ }^{12}$ Adapted from [8:23].
} 
defined by the nature of the harmonic terminations generated by the output matching network [16:28].

\subsubsection{Transconductance Amplifiers}

Transconductance amplifiers are assumed to generate output current waveforms that depend only on the input voltage waveform. In order to remain consistent with the assumption, the active device must operate solely in the saturation and cutoff regions at steady state. The proportion of the operation cycle during which the active device is in saturation, known as the conduction angle $(2 \alpha)$, defines the class of operation.

\subsection{Class A and B Amplifiers}

Class A amplifiers are operated entirely in the saturation region with a conduction angle of $2 \pi$ radians [11:447]. The input voltage and output voltage and current waveforms are shown against the load line in Figure 2.10 below.

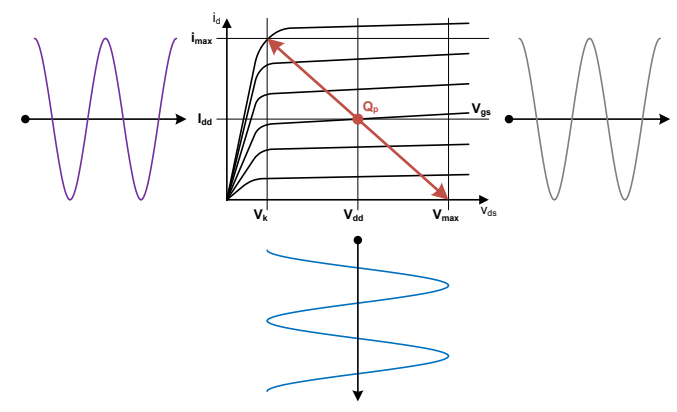

Figure 2.10: Class A load line (center) with input voltage (right) and output current (left) and voltage (bottom) waveforms.

Class B amplifiers are operated with a conduction angle of $\pi$ radians, equivalent to a $50 \%$ duty cycle in the saturation region. The input voltage and output voltage and current waveforms are shown against the load line in Figure 2.11 below. 


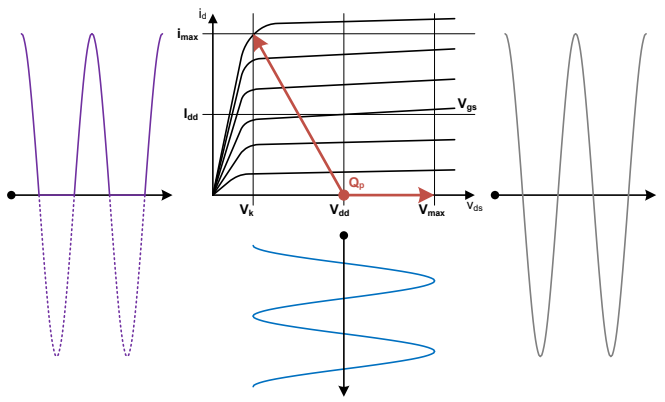

Figure 2.11: Class B load line (center) with input voltage (right) and output current (left) and voltage (bottom) waveforms.

While the class A amplifier is highly linear and will have minimal harmonic content, the maximum efficiency is $50 \%$ due to the overlapping regions in which the drain current and voltage are both non-zero [16:37]. In contrast, the class B amplifier has zero drain current for $50 \%$ of the operation cycle leading to a maximum efficiency of $78 \%$ at the expense of increased harmonic content and a large input amplitude requirement [11:451].

\subsection{Class $A B$ and $C$ Amplifiers}

When the active device is operated such that the conduction angle falls between the class $\mathrm{A}$ and $\mathrm{B}$ values, the result is referred to as a class $\mathrm{AB}$ amplifier. An example of a set of possible input voltage and output voltage and current waveforms are shown against the equivalent load line in Figure 2.12 below.

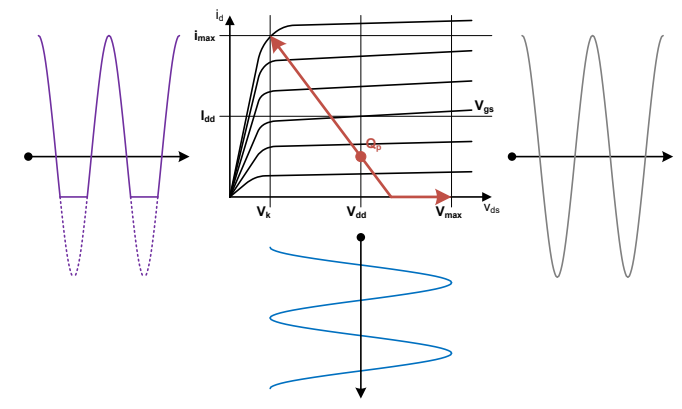

Figure 2.12: Class AB load line (center) with input voltage (right) and output current (left) and voltage (bottom) waveforms. 
Finally, when the active device is operated such that the conduction angle falls below the class B value of $\pi$ radians, the result is referred to as a class C amplifier. An example of a set of possible input voltage and output voltage and current waveforms are shown against the equivalent load line in Figure 2.13 below.

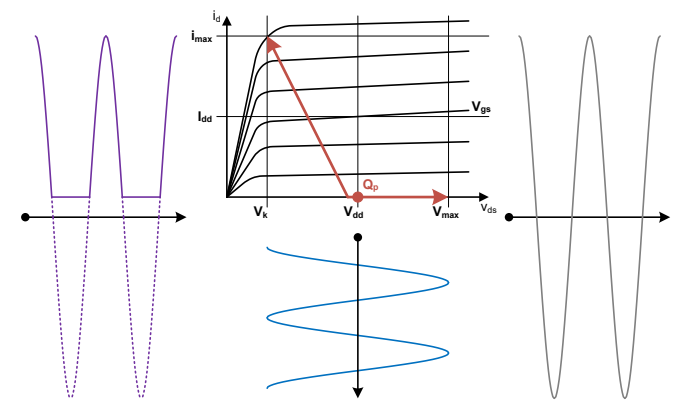

Figure 2.13: Class $\mathrm{C}$ load line (center) with input voltage (right) and output current (left) and voltage (bottom) waveforms.

The efficiency of the Class $\mathrm{AB}$ amplifier falls somewhere between class $\mathrm{A}$ at $50 \%$ and class B at $78 \%$ while retaining fairly strong linear performance [11:449]. As the conduction angle decreases in the class $\mathrm{C}$ amplifier, increased harmonic content is traded for increased efficiency.

\subsection{Summary}

The generalized equations for analyzing transconductance amplifiers are well documented in the literature [10]-[14], [16]. Using these equations, the maximum efficiency and normalized output power for the range of conduction angles can be calculated, shown here in Figure 2.14. 


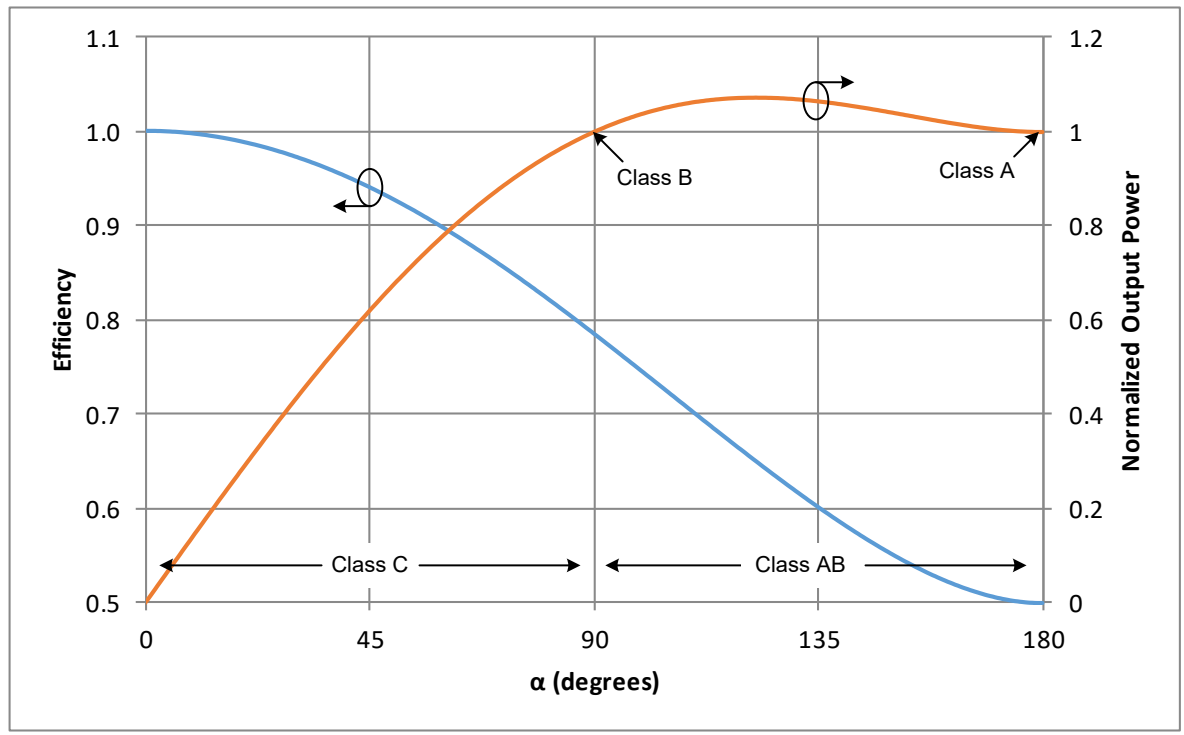

Figure 2.14: Efficiency and normalized output power v. $\alpha$ (degrees). ${ }^{13}$

\subsubsection{Switching Amplifiers}

Transconductance amplifiers improve efficiency by reducing the conduction angle to minimize the drain voltage and current overlap - thereby reducing the dc power dissipation through the active device. According to Tsai [16:62], the generation of these optimal current and voltage waveforms is particularly challenging when operating the active device as a high output impedance transconductor. Under this mode of operation, the drain voltage is controlled by two factors:

1. the harmonic composition of the drain current (defined by the non-linear performance of the active device); and,

2. the harmonic terminations of the output matching network.

Although it is relatively straight forward to control the latter through careful output network design, the required parameters are set by the former which will vary dramatically with input amplitude and active device bias.

\footnotetext{
${ }^{13}$ Adapted from [11:449-451]. The conductance angle is defined as $2 \alpha$.
} 
In contrast to transconductance amplifiers, switching amplifiers are assumed to generate output current waveforms that depend solely on the output matching network. In order to remain consistent with the assumption, the active device is operated as a switching device with a $50 \%$ duty cycle between triode and cutoff states [16:63]. While in the triode region, the active device shorts the drain voltage to the source - modelled with a small on-resistance, typically significantly less than the impedance of the output network. While in the cut-off region, the active device acts as an open circuit and the drain voltage is pulled to the rail.

Since the output signal amplitude is de-coupled from the input signal amplitude, the only input signal parameters carried from input to output are the frequency and phase modulation. Although this ensures switching operation, it restricts the utility of switching amplifiers to constant envelope input signals.

\subsection{Class D and $D^{-1}$ Amplifiers}

When operating the active devices as switches, there is the flexibility to either: a) switch the voltage and allow the output network to shape the current; or, inversely, b) switch the current and allow the output network to shape the voltage. This is best illustrated by considering the class $\mathrm{D}$ and inverse class $\mathrm{D}$ (or class $\mathrm{D}^{-1}$ ) amplifiers shown below. 
a)

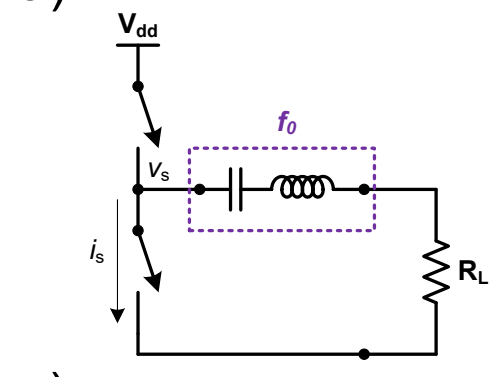

c)

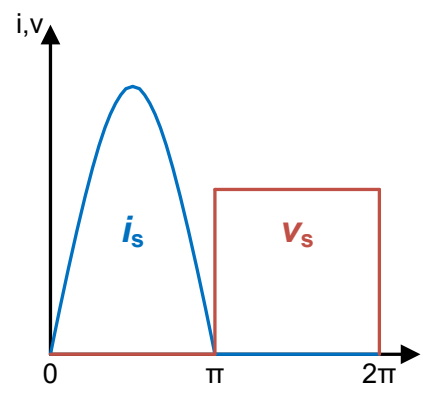

b)

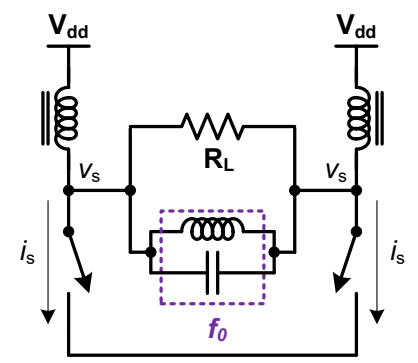

d)

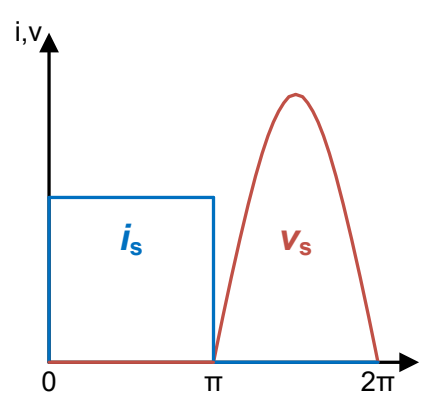

Figure 2.15: Class $\mathrm{D}$ and $\mathrm{D}^{-1}$ circuits (a and $\mathrm{b}$ ) and waveforms (c and d). ${ }^{14}$

The "voltage switching" class D amplifier (Figure 2.15a) alternately switches input of the load network from the supply voltage to ground. The series resonator before the load presents an open circuit to all frequencies except the fundamental. Practically, this circuit is infeasible at RF frequencies due to the difficulty of implementing the upper switching transistor and the frequency limiting behaviour of the drain-source capacitance [11:464]. The "current switching" class $\mathrm{D}^{-1}$ amplifier (Figure 2.15b) is a differential push-pull architecture that employs large RF chokes as current sources. The currents are alternately routed through the parallel connected load. The parallel resonator presents a short circuit to all frequencies except the fundamental, providing a low impedance bypass path to ground. Although, easier to realize than the nominal class D configuration; the inverse class D still has limitations due to the non-negligible drain-source

\footnotetext{
${ }^{14}$ Adapted from [17:52- 54].
} 
capacitances that hamper high frequency performance and the requirement for a synchronized differential drive signal [17:55].

\subsection{Class E Amplifiers}

The class E amplifier was first proposed by G. D. Ewing in 1964 [17:55] and resulted from an attempt to mitigate the presence of the parasitic drain-source capacitance by incorporating it into the output matching network. The canonical class E circuit and its current and voltage waveforms are shown in Figure 2.16 below.
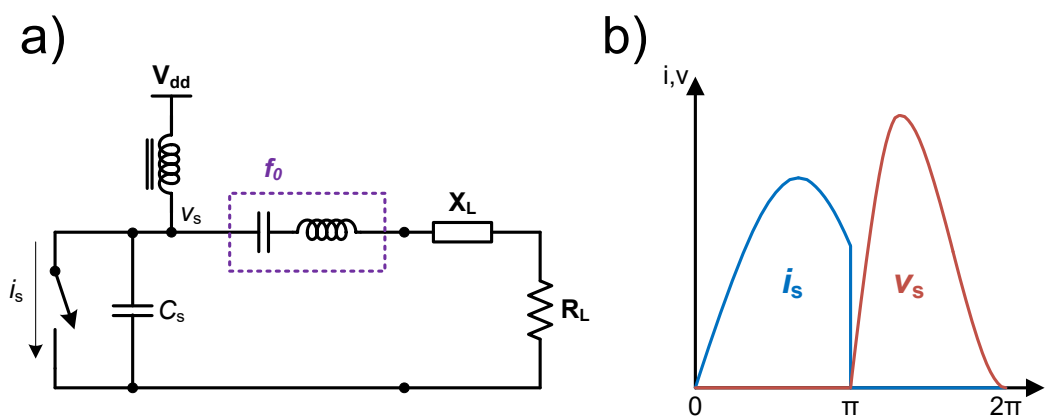

Figure 2.16: Class E circuit (a) and waveforms (b). ${ }^{15}$

Assuming the RF choke acts as an ideal current source and the series LC tank is tuned to the fundamental frequency with high $\mathrm{Q}$, a sinusoidal current will commutate between the switch (closed position) or shunt capacitance (open position). The tuning of the load reactance and shunt capacitance ensure the simultaneous zero voltage switching and zero voltage-slope switching conditions [17:57]. With ideal components, the theoretical efficiency of the class E amplifier is $100 \%$; with careful design, efficiencies of $80 \%$ are feasible [11:466].

\subsection{Class F Amplifiers}

Conceptually, it may be more appropriate to consider the class $\mathrm{F}$ amplifier as residing in the interstitial space between transconductance and switching amplifiers. The central conceit of

\footnotetext{
${ }^{15}$ Adapted from [17:58].
} 
the transconductance amplifier is that the output currents and voltages are represented by sinusoids (truncated or otherwise). This is realized in practice by ensuring that the active device load line remains entirely in the saturation or cutoff regions of operation. In contrast, the class F amplifier starts with the canonical class B amplifier and adds odd harmonics to the voltage waveform by adding harmonic terminations to the output matching network (Figure 2.17a).

a)

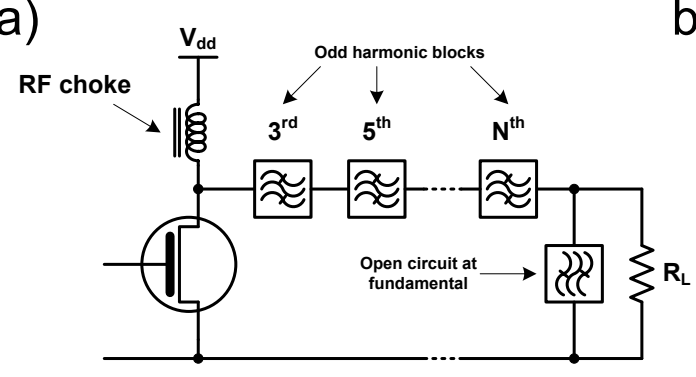

C) ${ }_{i_{d}, V_{d s}}$

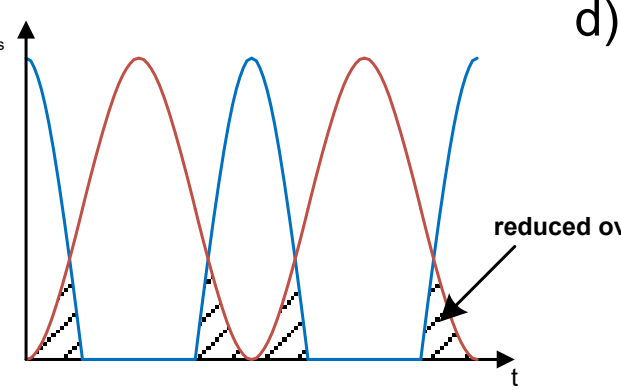

b)

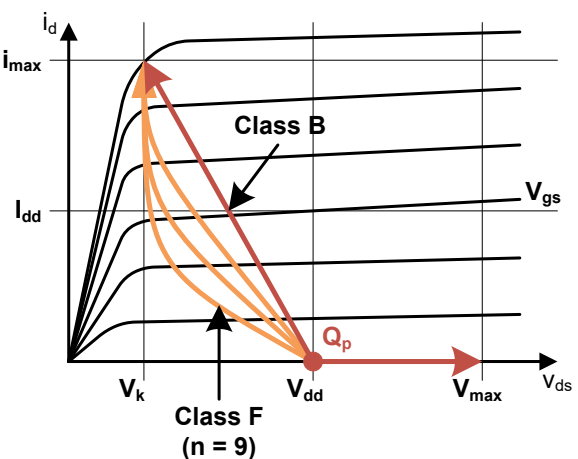

d)

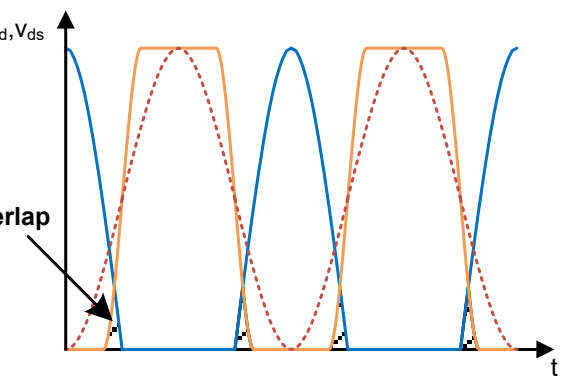

Figure 2.17: Class F amplifier circuit (a) alongside a comparison of class B and F load lines (b)

$$
\text { and waveforms (c and d). }{ }^{16}
$$

This has the effect of approximating a square wave [17:30] and pushing the load line towards the triode region where the active device acts more like a switch and less like a transconductor (Figure 2.17b). The square wave character of the drain voltage waveform minimizes the overlap region (Figure 2.17c and Figure 2.17d) during which the active device is conducting current with a non-zero voltage, thereby reducing power dissipation and improving

\footnotetext{
${ }^{16}$ Adapted from [17:52-54].
} 
efficiency. An additional advantage of the Class F harmonic tuning is that the maximum output power is improved without increasing the peak voltage of the waveform [17:33].

\subsubsection{Input and Output Networks}

The input source is connected through a passive network to the gate of the transistor. The function of this input network in a power amplifier is to achieve a low-loss impedance transformation for maximum power transfer from the source. This performance of the input network in terms of both matching and losses will have a major impact on the gain - and therefore, PAE - of the amplifier.

A second passive network connects the load to the drain of the transistor. The functions of this output network are twofold:

1. low-loss impedance transformation for optimal amplifier loading; and,

2. harmonic control determined by the desired mode of operation.

\subsubsection{Lumped-Element Matching Networks}

At RF frequencies ${ }^{17}$, wavelengths are on the order of several centimeters making transmission line matching difficult without requiring large die areas. Consequently, on-chip impedance matching networks at RF are typically implemented using lumped elements. Basic lumped-element matching networks take one of two basic L-network topologies (Figure 2.18, below) depending on the magnitude of the real part of the load respect to the desired input impedance.

\footnotetext{
${ }^{17}$ In concurrence with Cripps [8:23], "RF frequency", "dc current" and "ac current" will be used where appropriate for technical clarity.
} 

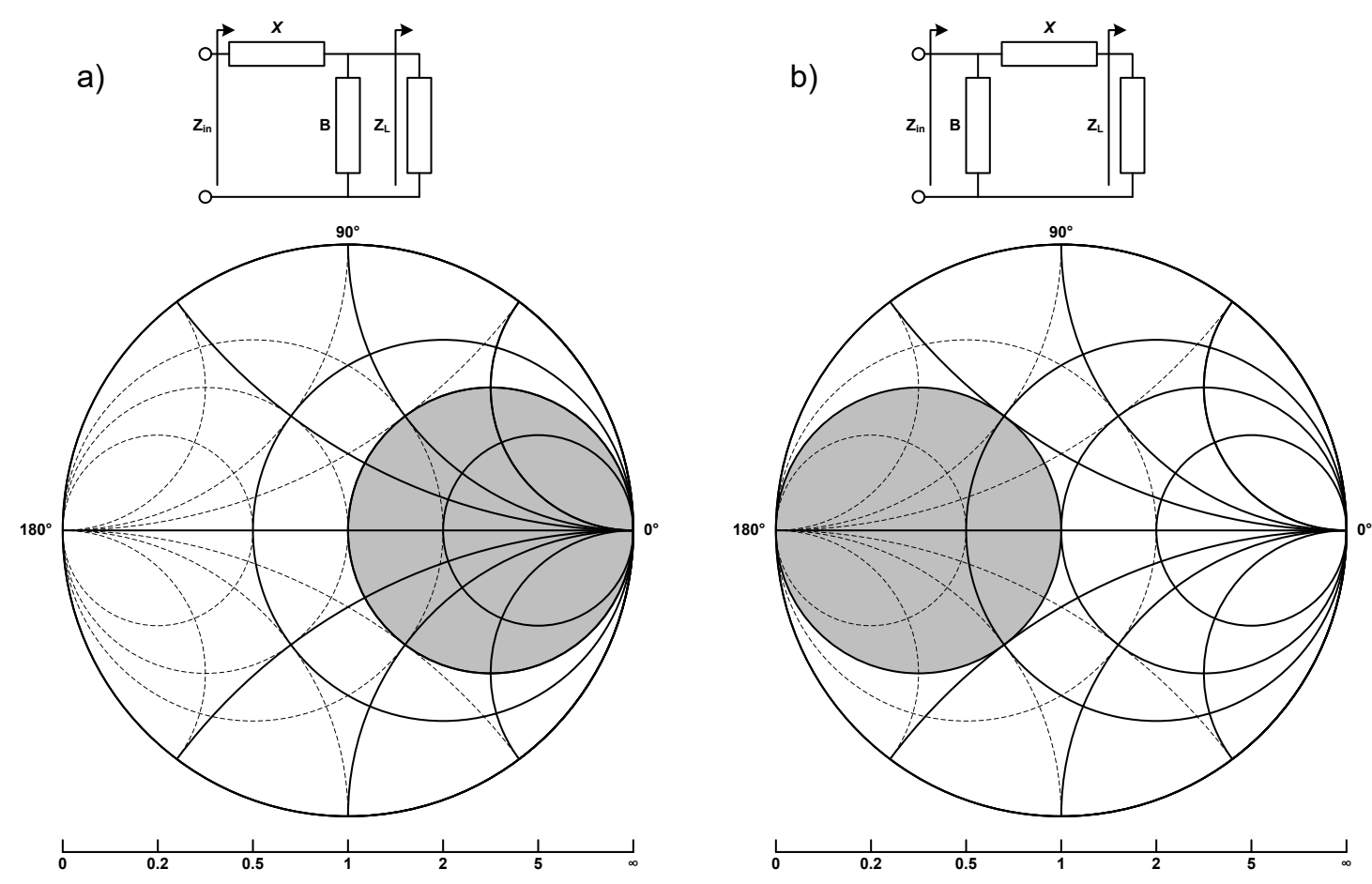

Figure 2.18: Basic lumped-element based impedance matching topologies. ${ }^{18}$

When matching load impedances located inside the $r=1$ circle, the left circuit (Figure 2.18a) must be used [11:107]. Conversely, when matching load impedances located inside the $g=1$ circle, the right circuit (Figure 2.18b) must be used [11:107]. These matching areas are indicated by the grayed out areas in the Smith charts below each network topology.

In general, the input of the amplifier will be driven by a signal carried on some transmission line of characteristic impedance, $Z_{0}$. In order to maximize power transfer, $Z_{\text {in }}$ must therefore have a real part equal to $Z_{0}$ and imaginary part equal to zero. The gate impedance of a power transistor is typically capacitive with minimal gate resistance to maximize $f_{\max }$. This will situate it in the lower left quadrant of the Smith Chart and therefore - assuming two element matching - the prototype circuit in Figure 2.18b should be used (as shown in Figure 2.19 below).

\footnotetext{
${ }^{18}$ Adapted from [11:107], [14:153].
} 


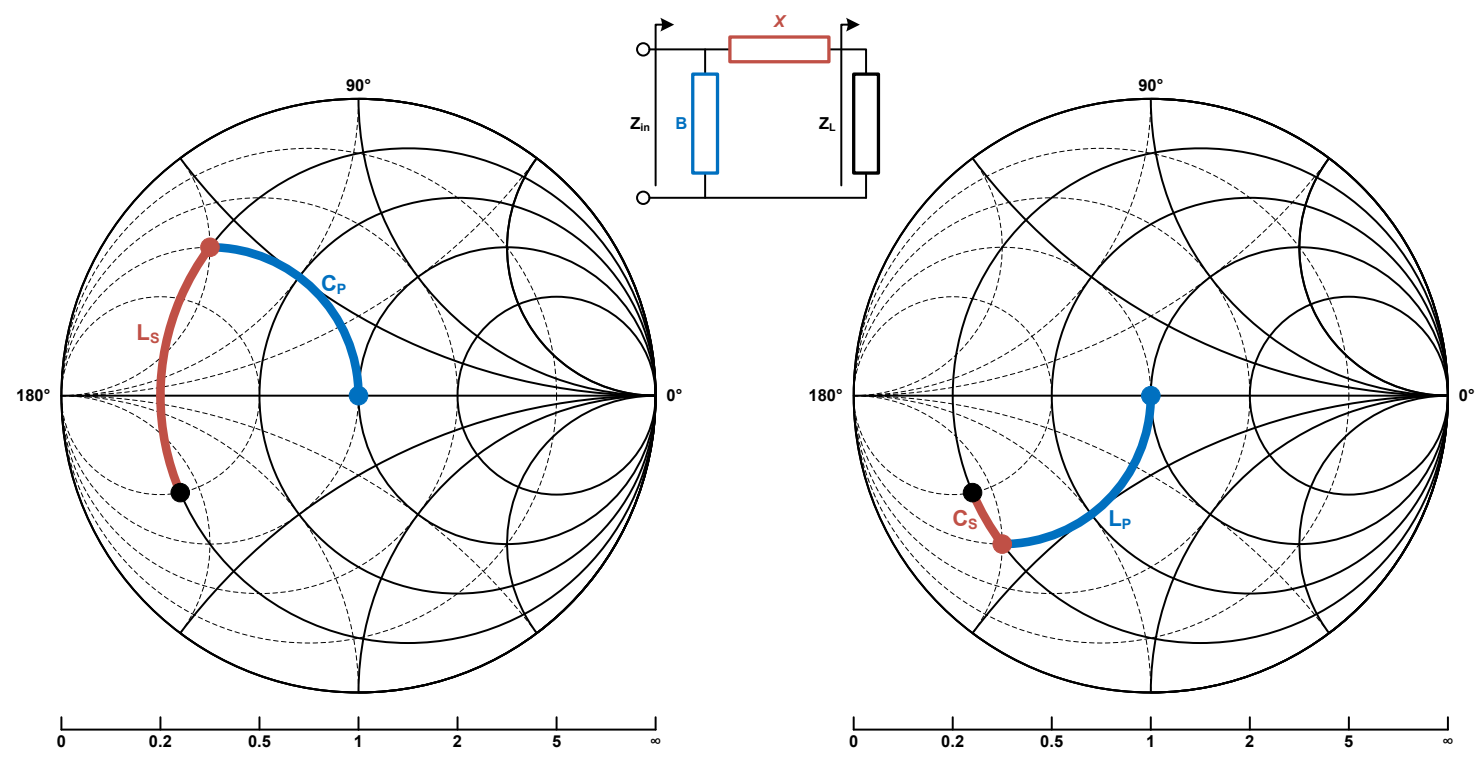

Figure 2.19: Two possible input matching networks for a typical power transistor. ${ }^{19}$

Another approach is to use an inductor connected in parallel with the gate input capacitance to resonate out the reactive component of the transistor input impedance. The subsequent real impedance can be matched using a conventional two element network (Figure 2.20).

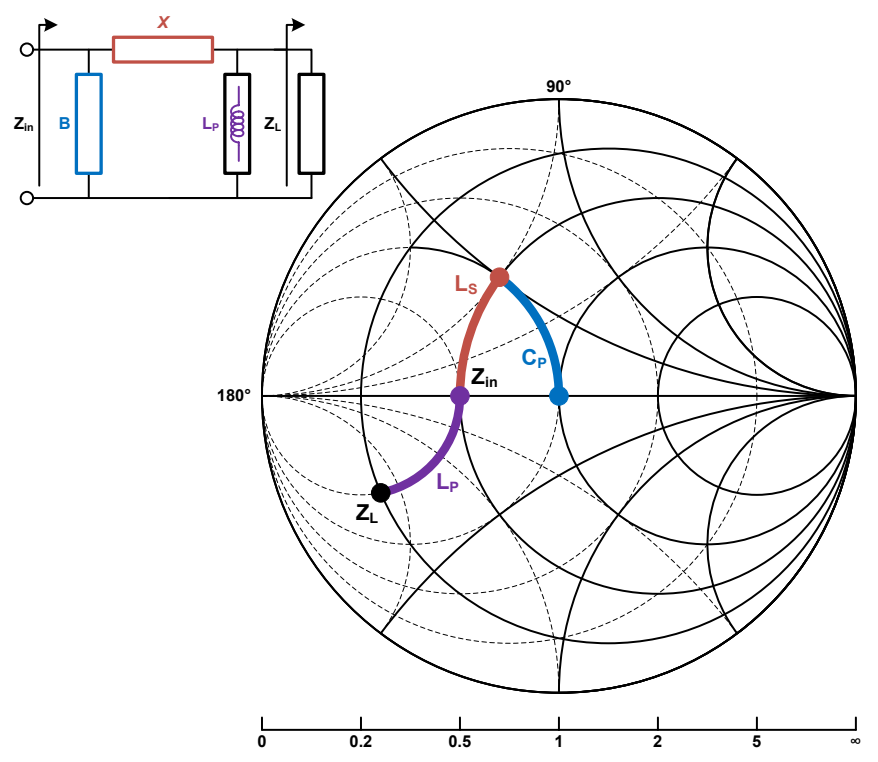

Figure 2.20: Example of input matching with a parallel inductance at the gate input. ${ }^{20}$

\footnotetext{
${ }^{19}$ Adapted from $[12: 16]$.

${ }^{20}$ Adapted from $[12: 16]$.
} 


\subsubsection{Magnetically Coupled Matching Networks}

Magnetic coupling can also be used to transform impedances through the use of on-chip transformers. The basic transformer structure and equivalent T-model are shown in Figure 2.21 below.
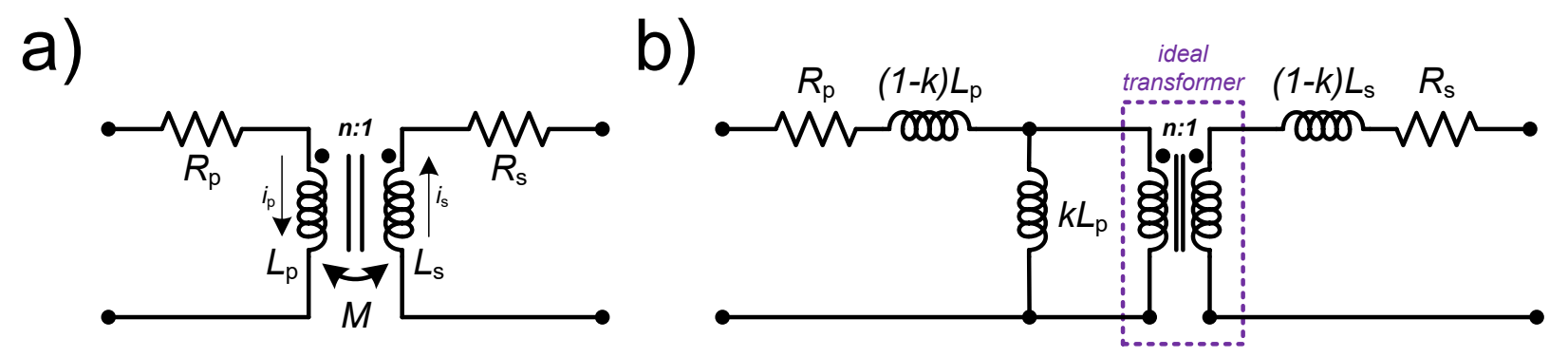

Figure 2.21: Basic transformer model (a) and equivalent T-model (b).

where the mutual inductance, $M$, and coupling factor, $k$, are related by the following expression [11:114]

$$
k=\frac{M}{\sqrt{L_{p} L_{s}}}
$$

For an ideal lossless transformer (i.e. $k=1, Q=\infty$ ), the impedance transformation is a function of the turns ratio, $n$; that is to say that the load impedance connected to the secondary side of the transformer appears $n^{2}$ times larger at the input to the primary [11:117]. In real transformers, the finite quality factor of the inductors will lead to parasitic resistances and a coupling factor somewhere between 0 and 1 .

A key consequence of low $k$-factors is the combination of significant attenuation and reduced bandwidth [18]. The leakage inductances form L-networks with the parasitic capacitances, creating undesirable impedance transformations. These can both be addressed to a certain degree with the inclusion of tuning capacitances at the primary and secondary (shown in Figure 2.22 below). 


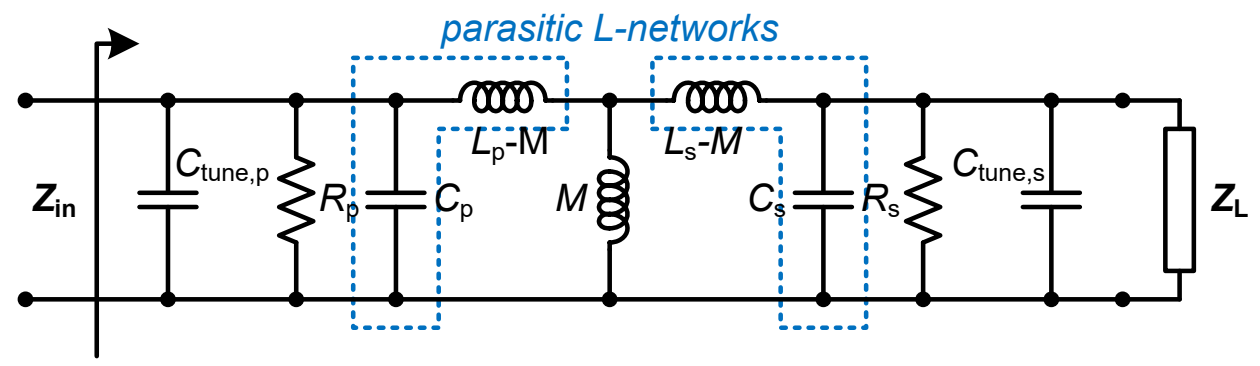

Figure 2.22: Transformer model showing parasitic capacitances generating lossy L-networks. ${ }^{21}$

Although resonant losses can be mitigated through appropriate tuning, a low $k$-factor also leads to large ohmic losses in the windings and therefore, even with tuning, $k$-factor should be maximized where possible [18].

\subsection{Chapter Summary}

In this chapter, a review of the silicon RF CMOS technology and transistor operation was conducted. The key PA performance parameters of power, gain, efficiency, linearity and stability were discussed. The general architecture of RF transistor amplifiers was presented and the transconductance and switching amplifier classes were summarized. Having established the theoretical foundations for power amplifier design, the next chapter will explore the distributed active transformer architecture developed by Ichoro Aoki in the early 2000s.

${ }^{21}$ Adapted from [18]. 


\section{Chapter 3: Distributed Active Transformer}

The DAT is a circuit architecture for high power fully integrated amplifiers that addresses the problems created by the low breakdown voltage and high loss integrated passives found in silicon processes [12]. The design incorporates six key features:

- $\quad$ push-pull component amplifiers establish a double differential drive that creates virtual ac grounds, avoiding the requirement for large integrated RF choke inductors and bypass capacitors [12:121];

- high quality factor slab inductors are used at the drain in order to minimize losses in the output network [12:129];

- circular geometry is used to connect adjacent transistor sources at virtual ac grounds, avoiding lengthy interconnects that would act as source degeneration inductances and otherwise degrade amplifier performance [12:131];

- cross connected drain capacitors are used to overcome the spatial separation of the component push-pull amplifier drains while maintaining harmonic suppression and wave shaping in the tuned load [12:133];

- transformer coupling is used to combine - in series - the output power of the low-voltage push-pull amplifiers while simultaneously reducing the load impedance (and therefore required voltage swing) of each [12:134]; and,

- cross connected gate inductors are used to adjust the low input impedance generated by the gate capacitance of the large power transistors in the component push-pull amplifiers and improve input matching [12:135]. 


\subsection{Double Differential Drive}

The central building blocks of the DAT architecture are push-pull amplifiers, shown with differential (Figure 3.1a) and transformer-coupled (Figure 3.1b) outputs below.
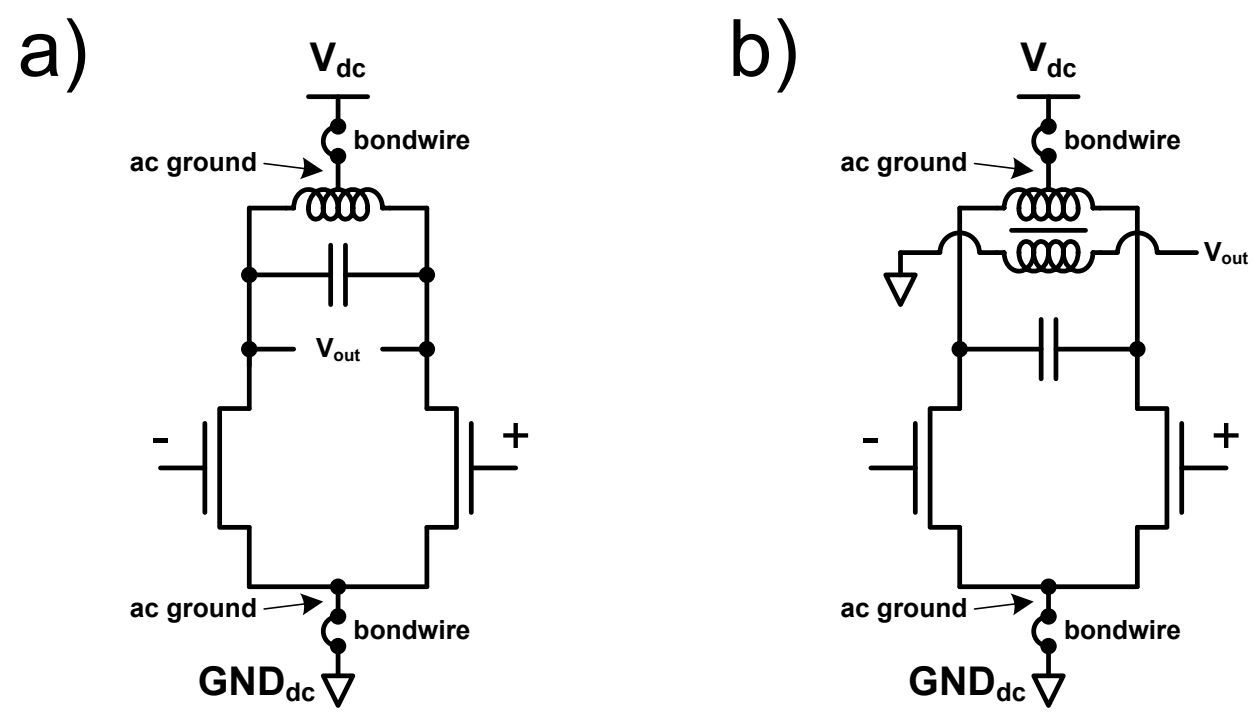

Figure 3.1: Push-pull amplifier with a) differential and b) transformer-coupled outputs. ${ }^{22}$

The differential signal across the push-pull pair creates virtual ac grounds at the plane of symmetry through the tuned load and source connections. These ac grounds only carry dc current and even harmonics [16:178], thereby minimizing loss at the fundamental frequency and simplifying the harmonic terminations required in the output matching network [12:122]. Furthermore, since the bond wires are connected across ac grounds, they do not experience ac currents at the fundamental frequency - eliminating the need for large on-chip bypass capacitors.

\subsection{Slab Inductors}

The second building block of the DAT are so-called "slab inductors" - essentially short microstrip transmission lines with largely inductive character. Figure 3.2 shows that starting

\footnotetext{
${ }^{22}$ Adapted from [12:122].
} 
from the ideal short circuit at the left, an ideal transmission line remains inductive so long as its length is less than $\lambda / 4$.

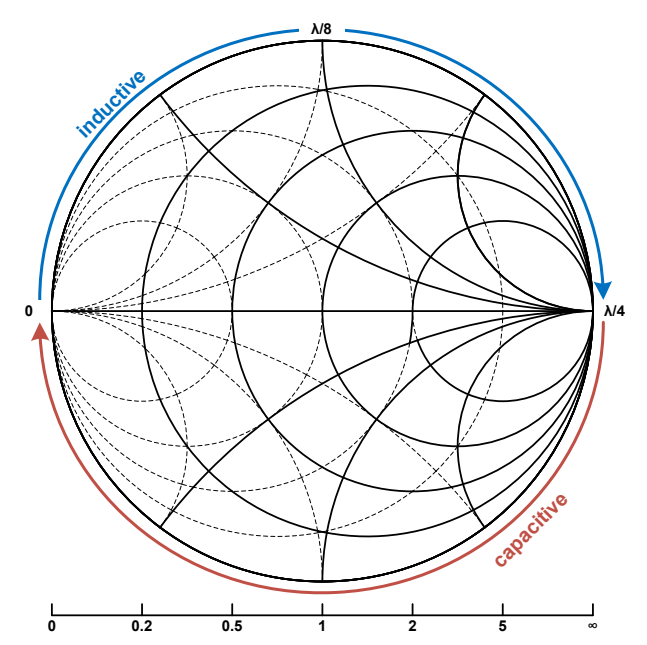

Figure 3.2: Smith chart showing inductive and capacitive regions.

The impedance of a very small transmission line terminated by an ideal short is given by

$$
Z_{\text {ind }}=Z_{o} \tanh (\gamma l) \approx Z_{0} \gamma l
$$

where $Z_{o}$ is the characteristic impedance, $\gamma$ is the complex propagation constant and $l$ is the line length. For microstrip, $Z_{o}$ is inversely proportional to the line width, $w$, and therefore inductance in slab inductors is improved with reduced width and increased length [12:126].

The quality factor of an inductor is the ratio of the energy its stores to the energy it dissipates and can be calculated as follows

$$
Q_{\text {ind }}=\frac{\operatorname{Im}\left(Z_{\text {ind }}\right)}{\operatorname{Re}\left(Z_{\text {ind }}\right)}
$$

Considering the classic lumped-element model for a transmission line below

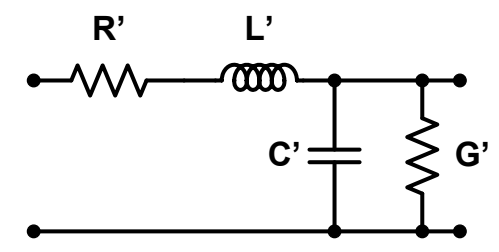

Figure 3.3: Lumped-element model of transmission line. 
$Z_{o}$ and $\gamma$ can be expressed in terms of the series impedance and shunt admittance per unit length, as follows

$$
\begin{gathered}
Z_{o}=\sqrt{\frac{R^{\prime}+j \omega L^{\prime}}{G^{\prime}+j \omega C^{\prime}}} \\
\gamma=\sqrt{\left(R^{\prime}+j \omega L^{\prime}\right)\left(G^{\prime}+j \omega C^{\prime}\right)}
\end{gathered}
$$

where $R^{\prime}$ and $L^{\prime}$ are the series resistance and inductance per unit length and $G^{\prime}$ and $C^{\prime}$ are the shunt conductance and capacitance per unit length, respectively.

Substituting (3.3) and (3.4) into (3.1), and applying the result to (3.2) gives

$$
Q_{\text {ind }} \approx \frac{\omega L^{\prime} l}{R^{\prime} l}=\frac{\omega L^{\prime}}{R^{\prime}}
$$

showing that quality factor is insensitive to $l$ and maximized by simultaneously increasing $L^{\prime}$ while decreasing $R^{\prime}$.

In a microstrip transmission line, $R^{\prime}$ decreases with increasing the line width however this has the effect of reducing the characteristic impedance and overall inductance of the slab inductor. Therefore, careful selection of $w$ and $l$ are required to maximize the $Q$ for a given required inductance.

In the original work on the DAT topology, Aoki et al. observed that - under certain conditions - slab inductors are favourable over spiral inductors because much higher quality factors can be achieved for the same inductance [12:129]. They attributed this to three key factors:

- first, spiral inductors will have a higher series resistance than equivalent slab inductors because the negative mutual inductance caused by currents in opposite sides of the spiral requires a longer length to achieve the same inductance value [12:129]; 
- second, the shunt resistance between the inductor terminals is increased because the terminals are farther apart, minimizing the substrate current and consequently the energy dissipation [12:129]; and,

- third, the metal width may be increased beyond what the maximum permissible width is for a spiral inductor thereby reducing the series resistance per unit length [12:130].

The DAT topology leverages high $Q$ slab inductors in the resonant load of the push-pull amplifiers outlined in section 3.1 where the combination of high quality factor and large line width minimize resistive losses and improve the efficiency of the amplifier.

\subsection{Circular Geometry and Cross Connected Drain Capacitors}

While the use of slab inductors dramatically improves the $Q$ of the tuned load, the large spatial separation of the inductor ports has two key consequences, specifically: how to a) establish the virtual ground at the transistor sources for the push-pull architecture and b) connect the load capacitor across the transistor drains without using long inductive interconnects (Figure 3.4).

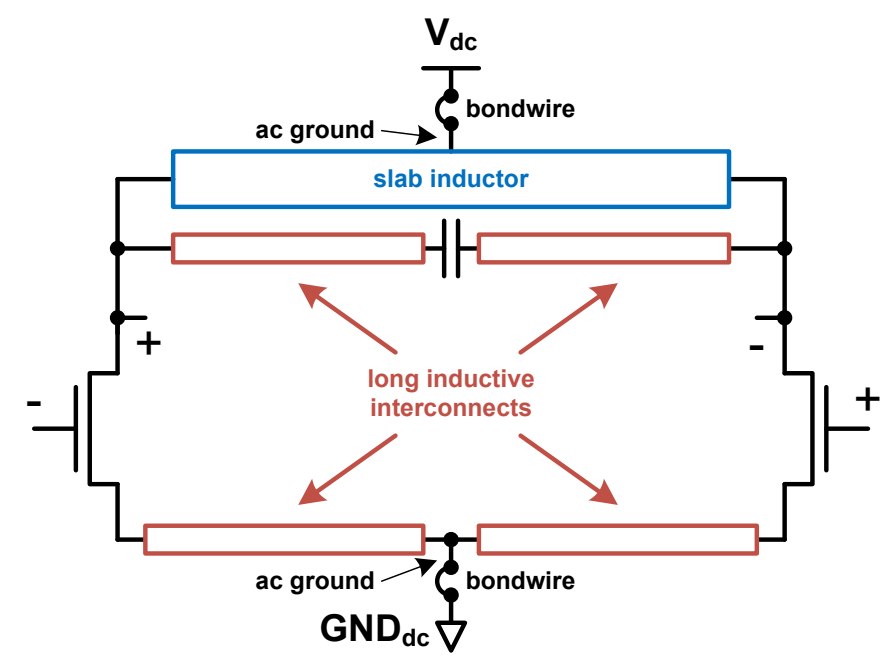

Figure 3.4: Push-pull amplifier with slab inductor and long interconnects. 


\subsubsection{Adjacent Source Connections}

From Section 3.1, a key advantage of the push-pull architecture is the creation of virtual ac grounds through the connection of the transistor sources. The use of the slab inductor across the drains of the push-pull pair creates a large distance between the transistor sources (Figure 3.4). If the sources are connected with metal interconnect, the inductance will be comparable to that of the slab inductor creating inductive source degeneration that will degrade the amplifier performance [12:31]. To avoid this, the DAT organizes four independent push-pull amplifiers in a circular layout, shown below:

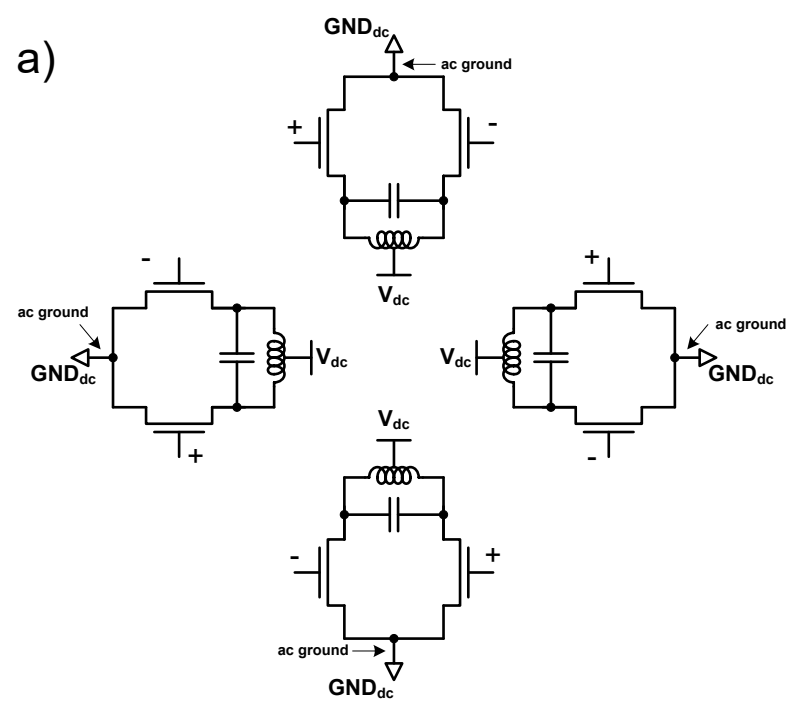

b)

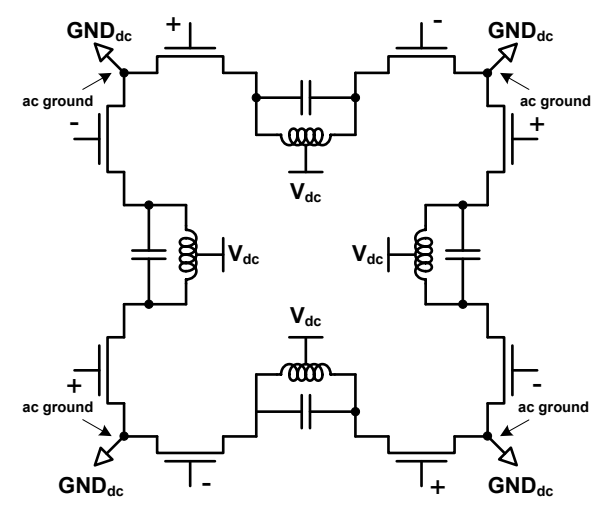

Figure 3.5: Circular geometry with a) conventional and b) adjacent source connections. ${ }^{23}$

By rotational symmetry, the geometry results in adjacent transistors being driven in opposite phase (Figure 3.5). Assuming every push-pull pair is driven with the same differential input signal, the source current for each transistor will be identical to its analog in every other pair. Consequently, a virtual AC ground can be established by connecting the transistor source to the nearest transistor source that is driven with opposite phase (Figure 3.5b); thereby avoiding long

${ }^{23}$ Adapted from [12: 132-133]. 
interconnects and maintaining a high quality harmonic termination to minimize AC losses $[12: 131]$

\subsubsection{Adjacent Drain Connections}

The load capacitor connection presents a similar problem to the above in that the spatial separation of the slab inductor ports leads to long interconnects (Figure 3.4) with inductances comparable to that of the load inductor itself [12:132]. The rotational symmetry of the layout conveniently resolves this issue by establishing an electrically equivalent node in close proximity to the transistor drain. Since the differential voltage and current experienced by the load capacitor under this connection scheme are equivalent to what it would experience in the conventional push-pull circuit, the harmonic termination and wave shaping are also identical [12:133]. The resultant circuit is shown in Figure 3.6 below.

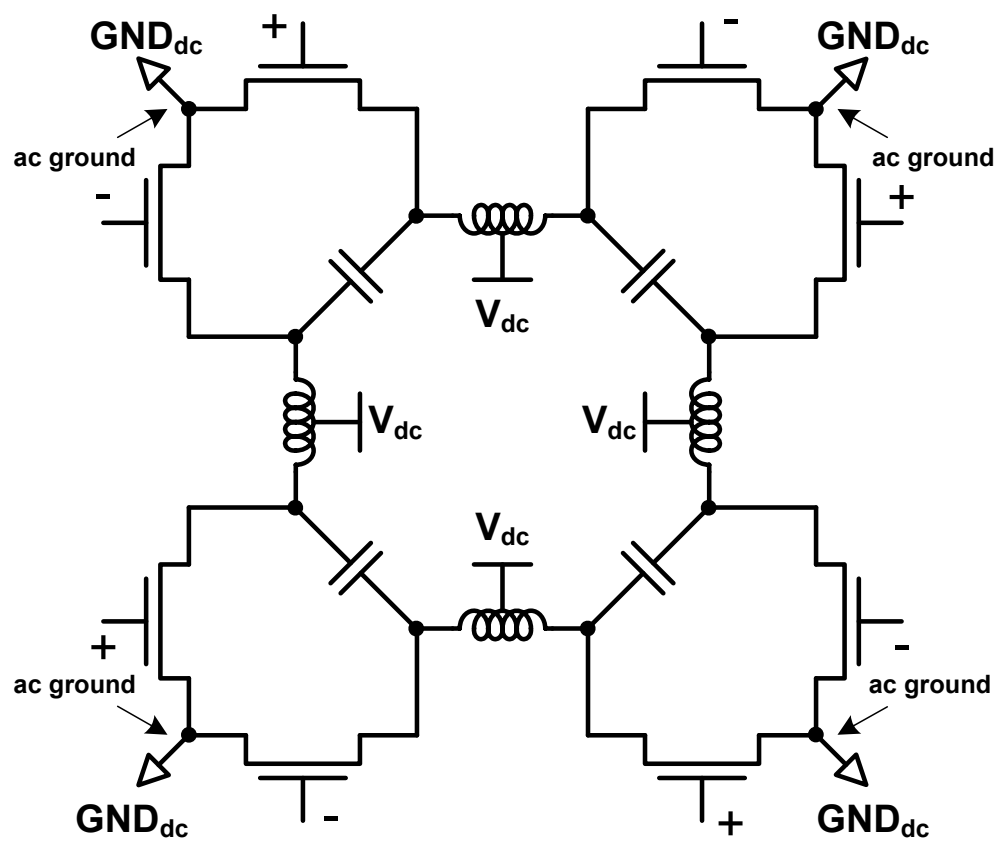

Figure 3.6: Circular geometry with adjacent source and drain connections. ${ }^{24}$

\footnotetext{
${ }^{24}$ Adapted from [12:133].
} 


\subsection{Transformer Coupling}

The DAT uses transformer coupling to combine the output powers of the unit push-pull amplifiers [12:134]. This is accomplished by placing a single turn spiral inside the circular layout to produce an output voltage from the induced magnetic fields generated by the slab inductors, as shown in Figure 3.7 below.

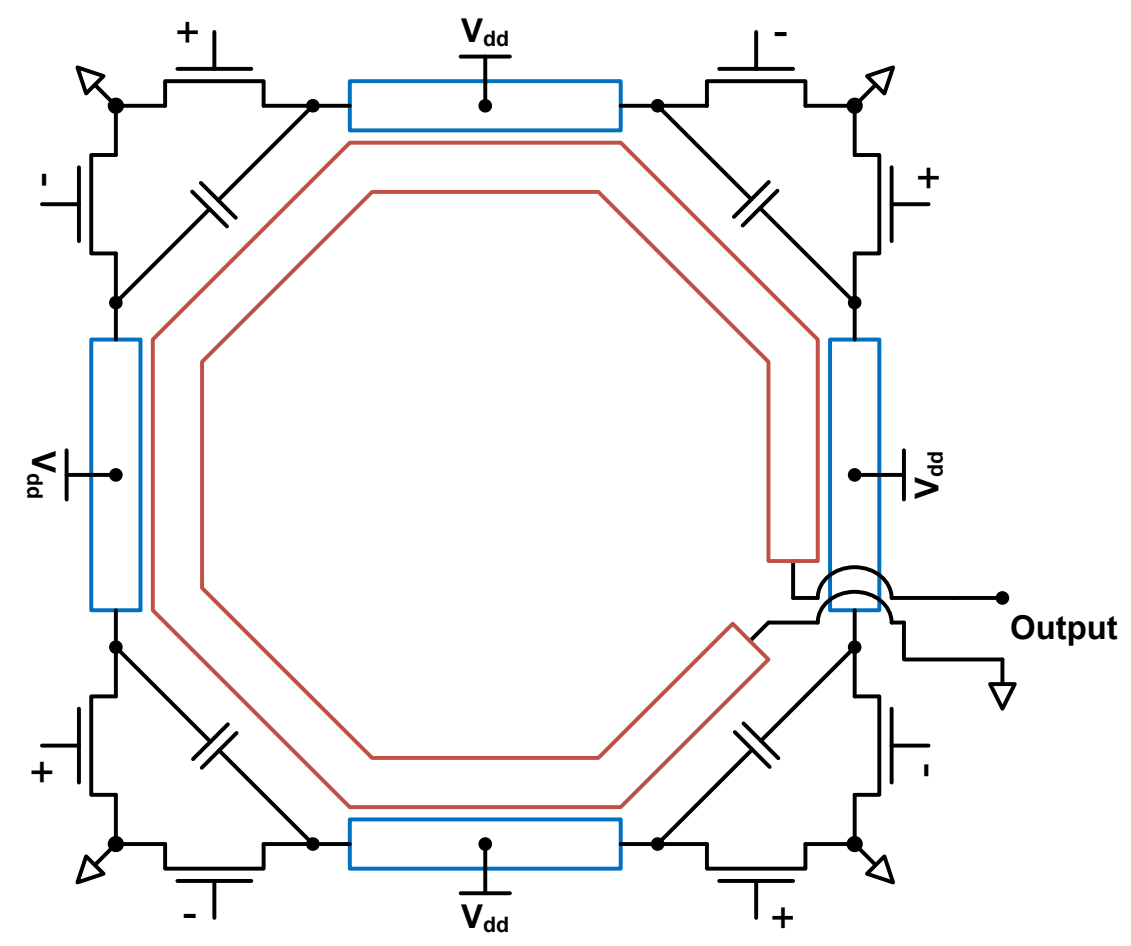

Figure 3.7: Circular geometry with slab inductors and transformer coupling. ${ }^{25}$

By the rotational symmetry of the DAT, each slab inductor conducts identical phase aligned AC currents. These AC currents generate magnetic fields that induce voltages along the coupled sections of the internal spiral whose magnitudes are defined by the equivalent turns ratio of the coupled line. Assuming each section is a 1:1 transformer, the voltage seen at the output is the

${ }^{25}$ Adapted from [12:135]. 
series combination of the differential voltages generated by each push-pull pair. In this context, the transformer acts as simultaneous power-combiner and impedance divider [12:134].

\subsection{Cross Connected Gate Inductors}

High power applications require large transistors which pose difficulties when matching to $50 \Omega$ sources due to the presence of a very large gate capacitance [12:134]. Using a conventional two-element network would require a very large series inductance that would difficult to implement on chip. The DAT topology addresses this by using the parallel inductance method discussed in Section 2.5.2.1. A cross-connected inductor is placed in parallel with the gate to resonate out the reactive contribution of $C_{g s}$ and bring the input closer to the center of the Smith chart. The cross connection between adjacent opposite phase transistors establishes a virtual $\mathrm{AC}$ ground at the midpoint of the inductor that removes the requirement for an on-chip bypass capacitor, as illustrated in Figure 3.8 below.

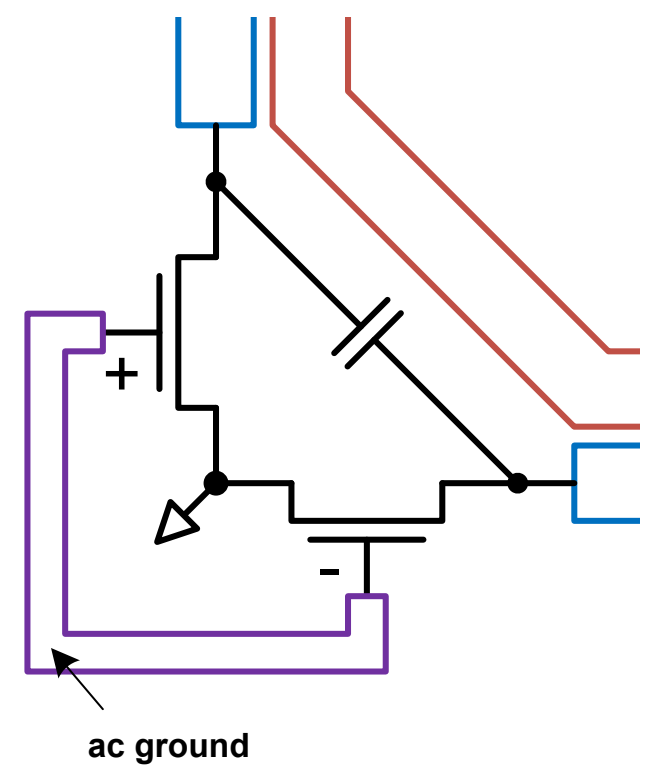

Figure 3.8: Cross connected gate inductor and associated AC ground. ${ }^{26}$

${ }^{26}$ Adapted from [12:136]. 


\subsection{Chapter Summary}

In this chapter, the distributed active transformer topology was introduced and the fundamental design features of differential drive, slab inductance and cross-connected reactance were discussed. In the next chapter, the concept of injection locking will be presented; which, together with DAT, forms the conceptual basis for the proposed amplifier design. 


\section{Chapter 4: Injection Locking}

Injection-locking is a phenomenon in which an injected signal pulls an otherwise selfoscillating circuit to the signal frequency [11:376]. This phenomenon can be leveraged to improve the gain - and subsequently, the PAE - of power amplifiers by decoupling the large power transistors with high input capacitances from the input signal [16:167], [19]. The input signal is instead used to drive small injection transistors that pull a power oscillator to the desired frequency. By reducing the size of the transistors seen by the input signal, the required voltage amplitude and consequently, required input power - can be reduced while maintaining the same output power, thereby improving the gain. Figure 4.1 illustrates how a push-pull amplifier and a $-g_{m}$ oscillator can be combined to form an injection-locked differential amplifier.
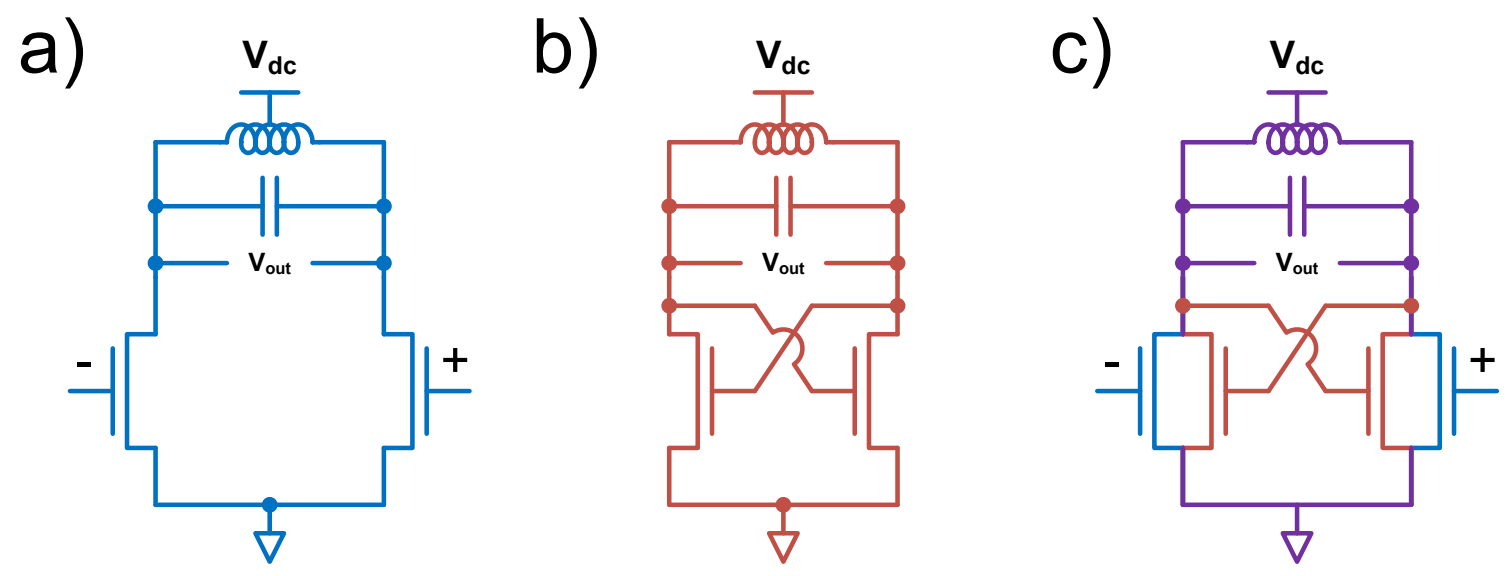

Figure 4.1: A push-pull amplifier (a), $-\mathrm{g}_{\mathrm{m}}$ oscillator (b) and injection-locked amplifier (c).

The push-pull amplifier was introduced in Chapter 3 , whereas the $-g_{m}$ oscillator and injection locking techniques will be discussed in the following sub-sections. 


\subsection{Fundamentals of Oscillator Design}

The $-g_{m}$ oscillator consists of two blocks: an LC resonator and a $-g_{m}$ cell. The canonical differential architecture (in which the $-g_{m}$ cell is implemented as a cross coupled differential pair) and its DC and RF equivalent circuits are shown in Figure 4.2 below.
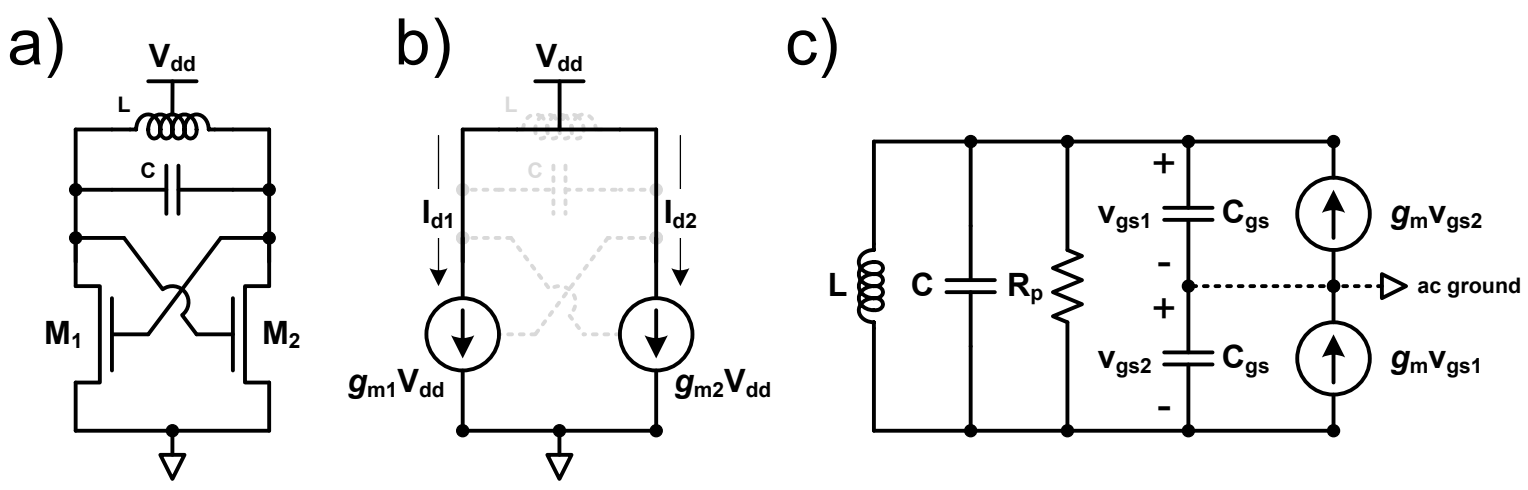

Figure 4.2: Simplified $-\mathrm{g}_{\mathrm{m}}$ oscillator (a) with DC (b) and RF (c) equivalent circuits.

The frequency of oscillation is set by the LC resonator and can be determined using the following equation [11:292]

$$
\omega_{o}=\frac{1}{\sqrt{L C}}
$$

When stimulated with an impulse, an ideal LC resonator will ring indefinitely at the frequency of oscillation. However, the finite quality factors of the inductor and capacitor in the resonator lead to parasitic resistances that dampen the oscillations, eventually decaying to zero.

A feedback loop can be used to introduce a negative resistance (in the form of a $-g_{m}$ cell) that overcomes the parasitic losses in the resonator; such a system is typically analyzed in the frequency domain using the feedback control system model shown in Figure 4.3 below: 


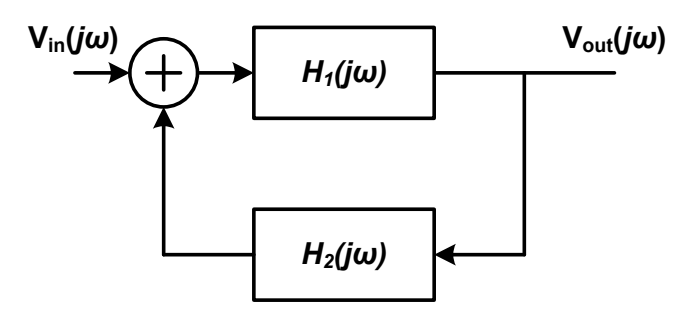

Figure 4.3: Open-loop model of an oscillator as a feedback control system. ${ }^{27}$

The open loop gain of the system is expressed as follows

$$
\frac{V_{\text {out }}(j \omega)}{V_{\text {in }}(j \omega)}=\frac{H_{1}(j \omega)}{1-H_{1}(j \omega) H_{2}(j \omega)}
$$

In order for the system to oscillate, the poles of the system must fall on the $j \omega$ axis. This can be established so long as the following conditions are satisfied (referred to as the Barkhausen criterion). First, the magnitude of the loop gain must be equal to one, as shown below

$$
\left|H_{1}(j \omega)\right|\left|H_{2}(j \omega)\right|=1
$$

and second the aggregate phase around the loop is some multiple of $2 \pi$, as shown below

$$
\angle H_{1}(j \omega) H_{2}(j \omega)=2 \pi n
$$

where $n \geq 0$ and $n \in \mathbb{Z}[11: 294]$.

\subsection{Injection Locking Technique}

The $-g_{m}$ oscillator in Figure 4.2 can be extended to include injection locking circuitry as shown in Figure 4.4a below.

\footnotetext{
${ }^{27}$ Adapted from [11:294].
} 

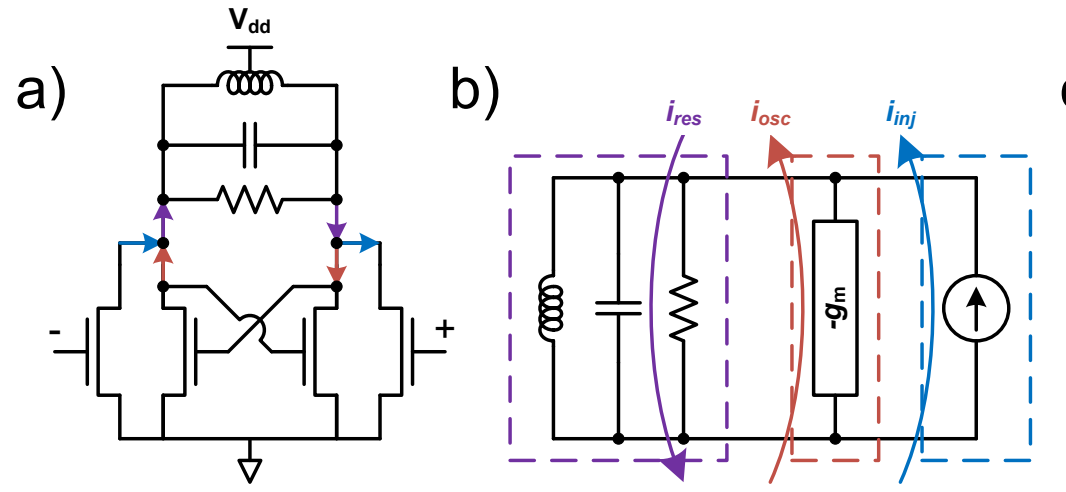

c)

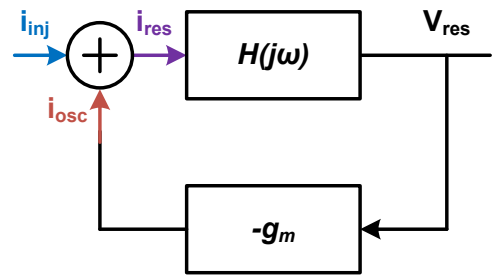

Figure 4.4: Injection-locking circuit (a) with functional block organization (b) and equivalent feedback system.

Current injection at a frequency close to the natural oscillating frequency of the circuit causes a phase shift between the resonator and oscillator current. From the closed loop analysis in Figure $4.4 \mathrm{c}$, the steady state resonator current is the phasor combination of the injection and oscillator currents. This relationship is illustrated in Figure 4.5a below.
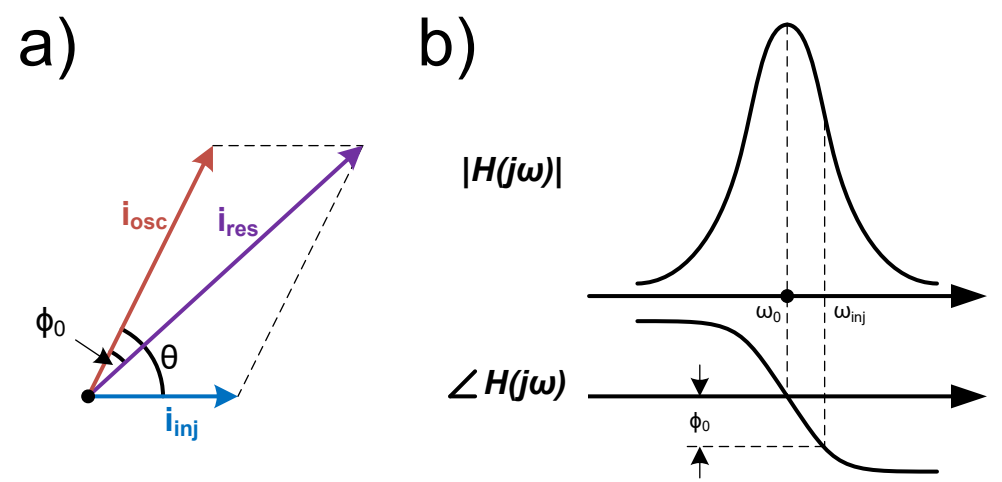

Figure 4.5: Phasor representation of oscillator currents under injection (a) and open loop characteristics of the resonator (b). ${ }^{28}$

The steady state phase difference between the injection and resonator currents is given by the following expression [20]

\footnotetext{
${ }^{28}$ Adapted from [20].
} 


$$
\sin \theta=\sin \phi_{0} \cdot \frac{i_{r e s}}{i_{i n j}}
$$

where $\phi_{0}$ is the phase difference between the oscillator and resonator currents. This phase difference is determined by the phase characteristics of the resonator at the injection frequency (Figure 4.5b) and can be reasonably approximated as follows [20]

$$
\tan \phi_{0} \approx \frac{2 Q_{L}}{\omega_{0}} \cdot\left(\omega_{0}-\omega_{\text {inj }}\right)
$$

\subsection{Determining Locking Range}

The locking range defines the maximum frequency offset of the injected signal relative to the nominal self-oscillation frequency of the resonator for which the oscillator will lock. The locking range is dependent on both the quality factor of the tank circuit and ratio of injected current to oscillating current. Through some manipulation of (4.5) and (4.6), an expression for the locking range can be determined, as shown below [20]

$$
\omega_{o}-\omega_{i n j}=\frac{\omega_{o}}{2 Q} \cdot \frac{I_{i n j}}{I_{\text {osc }}} \cdot \frac{1}{\sqrt{1-\frac{I_{\text {inj }}^{2}}{I_{\text {oSc }}^{2}}}}
$$

\subsection{Chapter Summary}

In this chapter, the concept of injection locking was introduced. A self-oscillating circuit was analyzed and extended to include injection circuitry. The injection-locking phenomenon was reviewed and the analytical expression for locking range and its derivation was discussed. Using the theory established in the previous chapters, the design and implementation of the novel injection-locked DAT amplifier will now be presented. 


\section{Chapter 5: Design and Implementation}

This chapter discusses the design and implementation of the integrated power amplifier beginning with the specification and constraints followed by the conceptual circuit organization and floorplan. Next, the detailed design and layout descriptions for each sub-circuit are provided. The chapter concludes with a discussion of the final iteration and simulation results. As the design process was highly iterative, the design methodology presented covers the initial iteration of each module and sub-circuit. The final iterations are used when discussing the layouts and simulation results.

\subsection{Specification \& Constraints}

A specification was developed to guide the design optimization and constrain the design space. The key parameters are provided in Table 5.1 below.

Table 5.1: Power Amplifier Specification

\begin{tabular}{clrc}
\hline Ser & Parameter & Value Units & Tolerance \\
\hline \hline 1 & Supply Voltage $\left(V_{D D}\right)$ & $1.5 \mathrm{~V}$ & $\leq$ \\
2 & Center Frequency $\left(f_{c}\right)$ & $2.4 \mathrm{GHz}$ & - \\
3 & Bandwidth $(\Delta f)$ & $500 \mathrm{MHz}$ & $\geq$ \\
4 & Output Power $\left(P_{\text {out }}\right)$ & $+27 \mathrm{dBm}$ & - \\
5 & Gain $(G)$ & $10 \mathrm{~dB}$ & $\geq$ \\
6 & Power Added Efficiency $(\mathrm{PAE})$ & Maximum $\%$ & - \\
7 & I/O Match & \\
8 & Chip Area & $50 \Omega$ & - \\
\hline
\end{tabular}

${ }^{29}$ The I/O match is single-ended. 
All specifications were selected by the author with the exception of the specified chip area requirement. This requirement was determined by the maximum granted die area from the fabrication application process through CMC.

\subsection{Conceptual Design}

The conceptual design originated from the recognition that the logic that allowed the crosscoupling of the tank capacitors in the push-pull amplifier within the DAT could be applied to any differential pair circuit. Although the DAT generates large output powers, it was hypothesized that efficiency could be improved through gain enhancement. The circuit architecture initially proposed and implemented by Aoki [12] for an eight device DAT was adapted to use injectionlocked power oscillators (Figure 5.1).

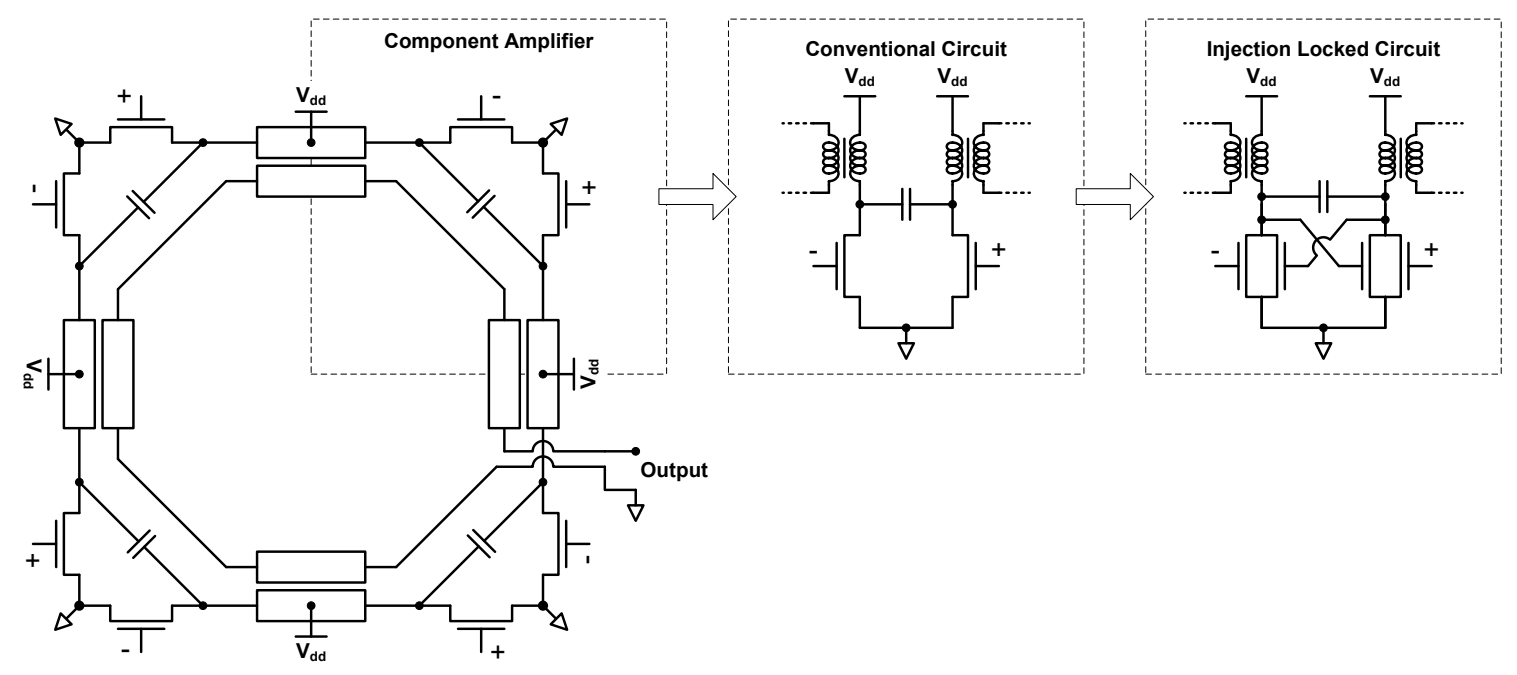

Figure 5.1: Design evolution from conventional DAT to injection-locked DAT.

With this conceptual foundation, a modular topology was developed that organized the overall circuit into several smaller sub-circuits in order to simplify design and testing, as follows:

- the distributed active transformer module, which consists of: 
○ an output network of slab inductances, cross-coupled capacitors and output coupling loop that collectively provide both impedance matching and output transformer coupling; and,

- a set of four unit power cells that combine in the output network to provide the total output power.

- the input distribution network module, which consists of:

○ an integrated balun to generate a differential signal from the single-ended input; and,

- a set of two 1:4 power dividers that distribute the positive and negative signals from the balun to the inputs of the unit power cells.

This overall circuit organization is shown in Figure 5.2 below.

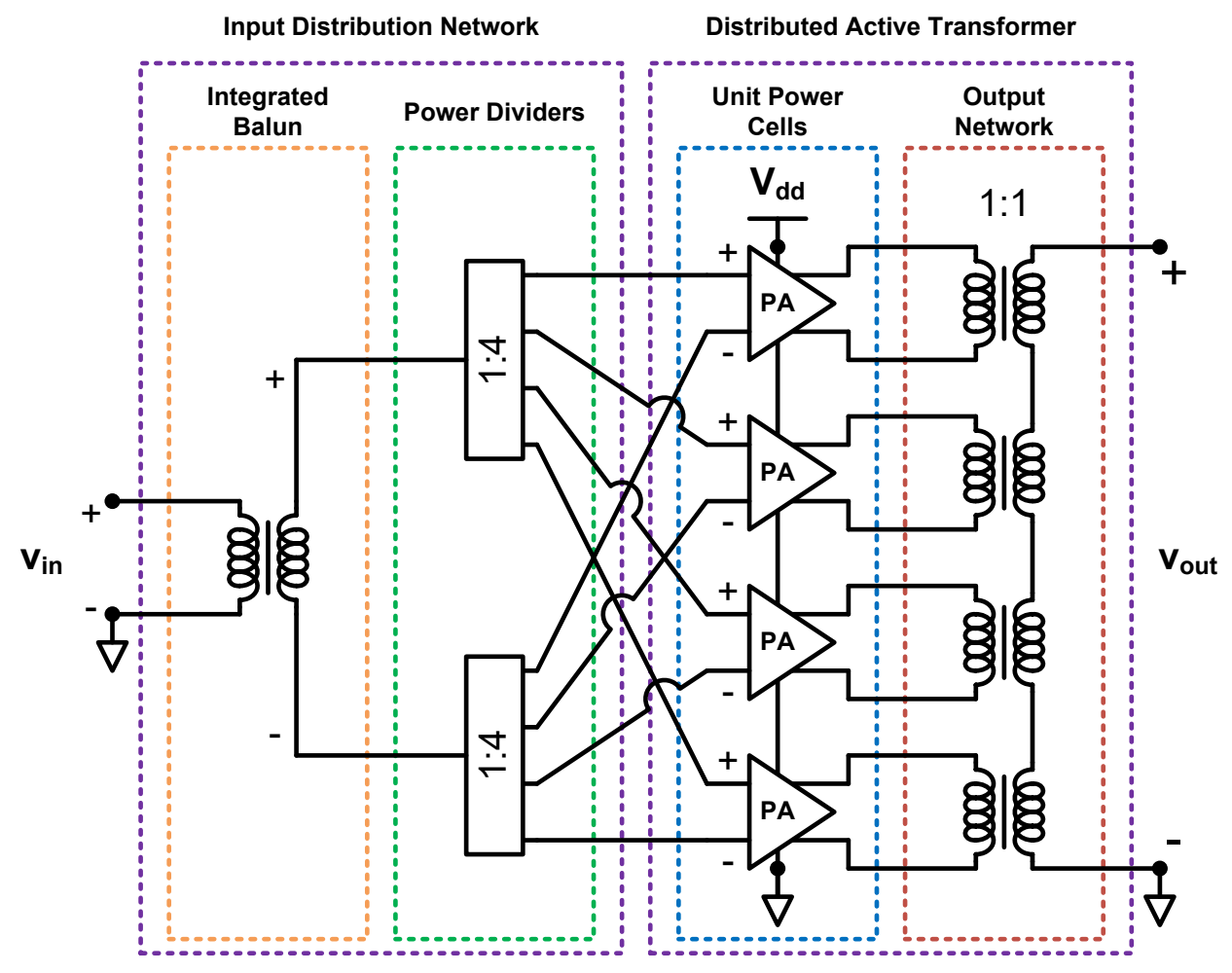

Figure 5.2: Modular topology for circuit design and layout. 
Using this modular organization, design iteration was completed as illustrated in the flow diagram below (Figure 5.3).

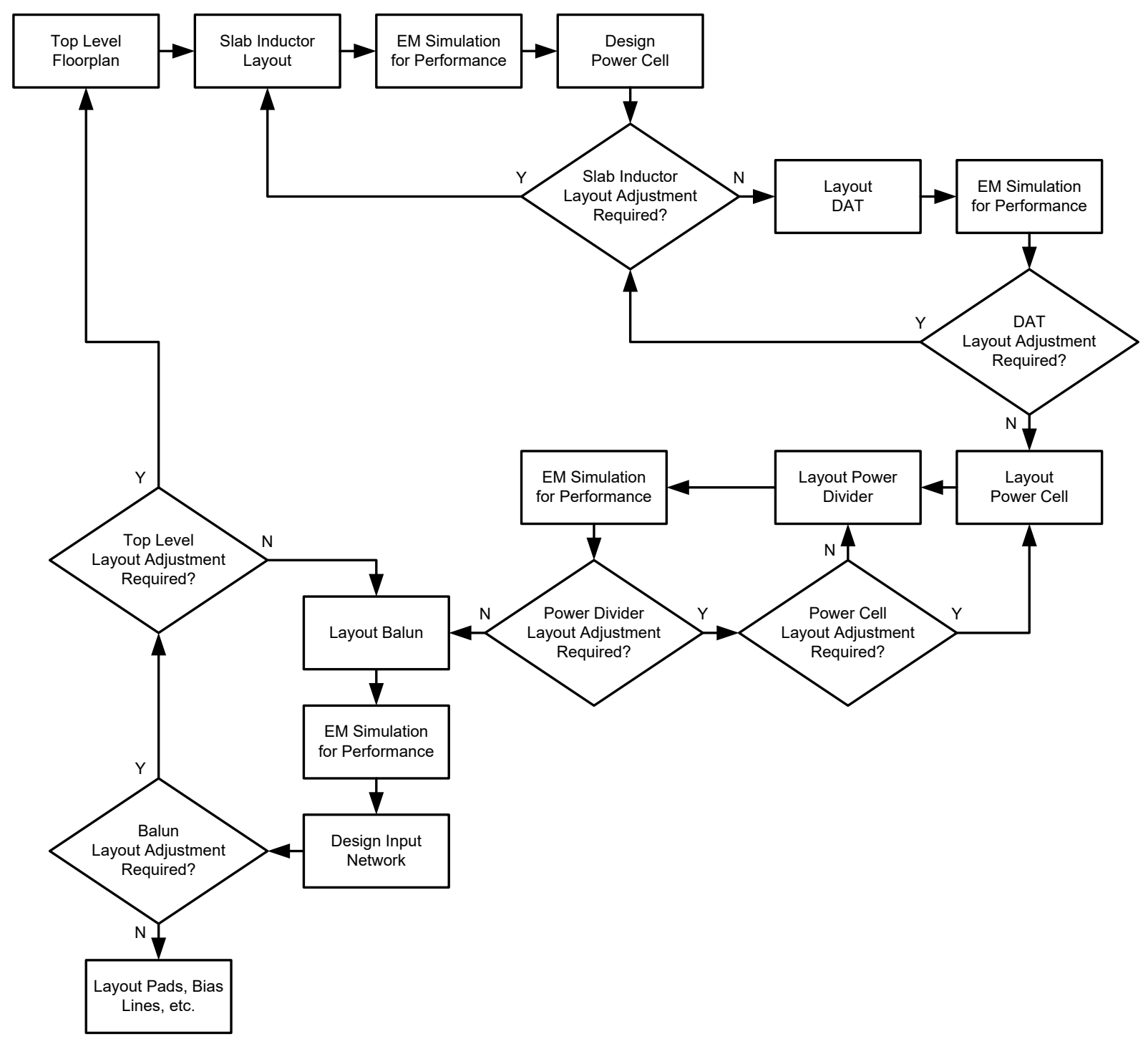

Figure 5.3: Iterative design flow diagram.

Due to the non-standard layout of the passive blocks, EM simulation was required to generate equivalent circuit models for performance testing; this simulation was completed using EMX ${ }^{\mathrm{TM}}$. 


\subsubsection{Floorplan}

The proposed design featured two sub-circuits which were expected to occupy significant circuit area: the integrated balun and the output network (due to the presence of several large slab inductances). In order to ensure that the design remained within the identified die area constraint (Table 5.1), some preliminary layout planning was required. The original DAT layout from Aoki [12] was used as a template (Figure 5.4) and an initial floorplan was developed (Figure 5.5).

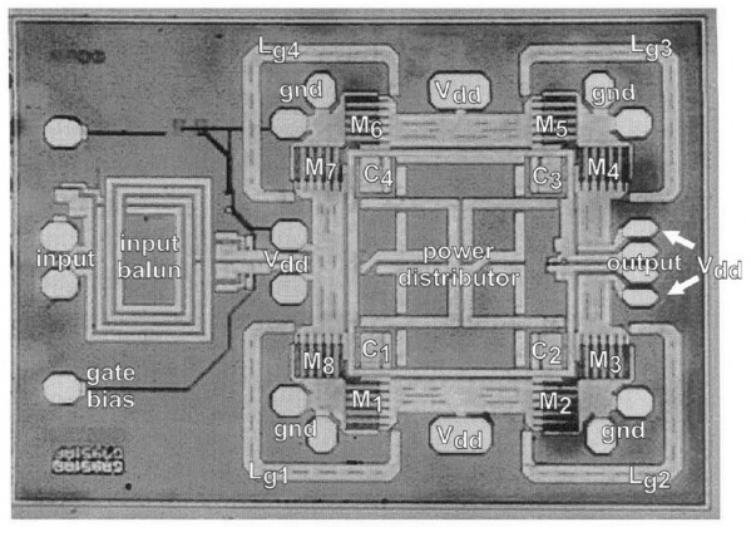

Figure 5.4: Template IC used for layout planning. ${ }^{30}$

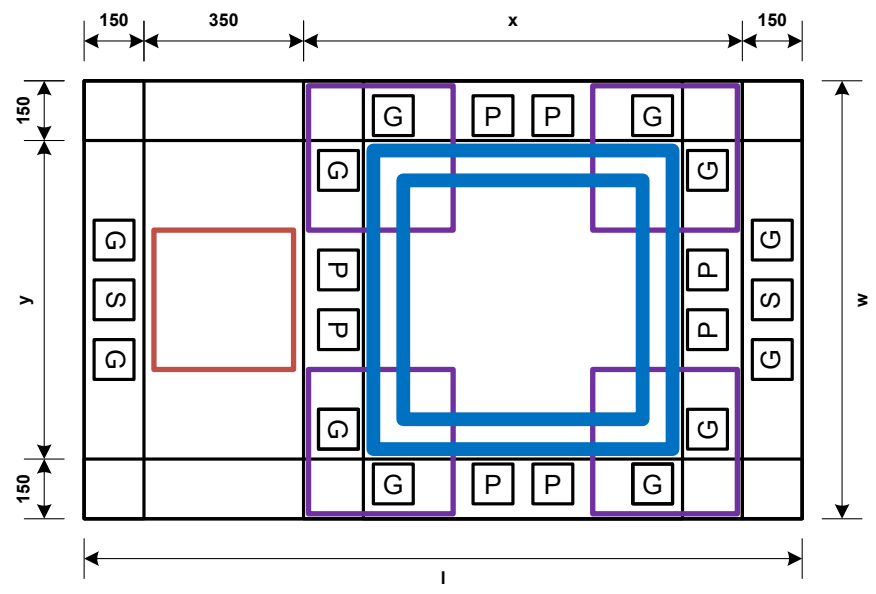

Figure 5.5: Initial floorplan with pad allocation.

\footnotetext{
${ }^{30}$ Reproduced from [12:168].
} 
First, a $150 \mu \mathrm{m}$ wide perimeter was allocated for GSG probe pads and the chamfer from the process chip ring. A preliminary design of the integrated balun indicated that $350 \mu \mathrm{m}$ would be sufficient. From these allocations and the area constraint, several equations were extracted:

$$
\begin{gathered}
l=x+350 \mu m+2 \cdot(150 \mu m) \\
w=y+2 \cdot(150 \mu m) \\
w \cdot l \leq 2 \mathrm{~mm}^{2}
\end{gathered}
$$

The DAT structure requires a square layout in order to establish the rotational symmetry that allows the cross-coupling of the load capacitors, therefore $x$ must be equal to $w$. Making the appropriate substitutions, the roots of resulting equation were determined

$$
\begin{gathered}
w^{2}+0.65 \cdot w-2=0 \\
(w-1.125)(w+1.775)=0
\end{gathered}
$$

Consequently, the preliminary width and length were set to $1.125 \mathrm{~mm}$ and $1.775 \mathrm{~mm}$, respectively; yielding a design area of $2 \mathrm{~mm}^{2}$ - consistent with the die area constraint in the specification.

\subsection{Distributed Active Transformer Design \& Layout}

Using the modular organization provided in Figure 5.2 as a guide, the DAT module was designed first. The DAT module consists of the unit power cells and output network - with a common interface through the slab inductors integral to the DAT topology. In this sub-section, the design of the slab inductances and unit power cells, and their subsequent integration and layout, are reviewed.

\subsubsection{Slab Inductor Design}

Having established a rough floorplan in Section 5.2.1, an estimate of the dimension constraints for the slab inductors was completed. Substituting $1.125 \mathrm{~mm}$ for $w$ in (5.2) and allowing for a small margin to accommodate design rule spacing requirements, the maximum outer 
diameter of the DAT was set to $800 \mu \mathrm{m}$. With this fixed outer diameter, the nominal length and maximum width of the slab are approximately related by the following formula

$$
\max \left(w_{\text {slab }}\right)=\frac{800 \mu m-l_{\text {slab }}}{2}
$$

Given a slab inductor is effectively a short section of transmission line terminated by a short to ground [12:125], the design kit transmission line component was used as a template to develop the slab inductors used in the final design. A simulation was used extracting the expected inductance and quality factor at the design frequency of $2.4 \mathrm{GHz}$ for various slab dimensions. A set of rough layout iterations established upper and lower bounds for the length at 700 and 400 $\mu \mathrm{m}$, respectively. The slab length and width were swept parametrically, with the width limited in each sweep to a maximum as defined by (5.6) and an minimum of $50 \mu \mathrm{m}$. The results of that analysis are shown in the figures below.

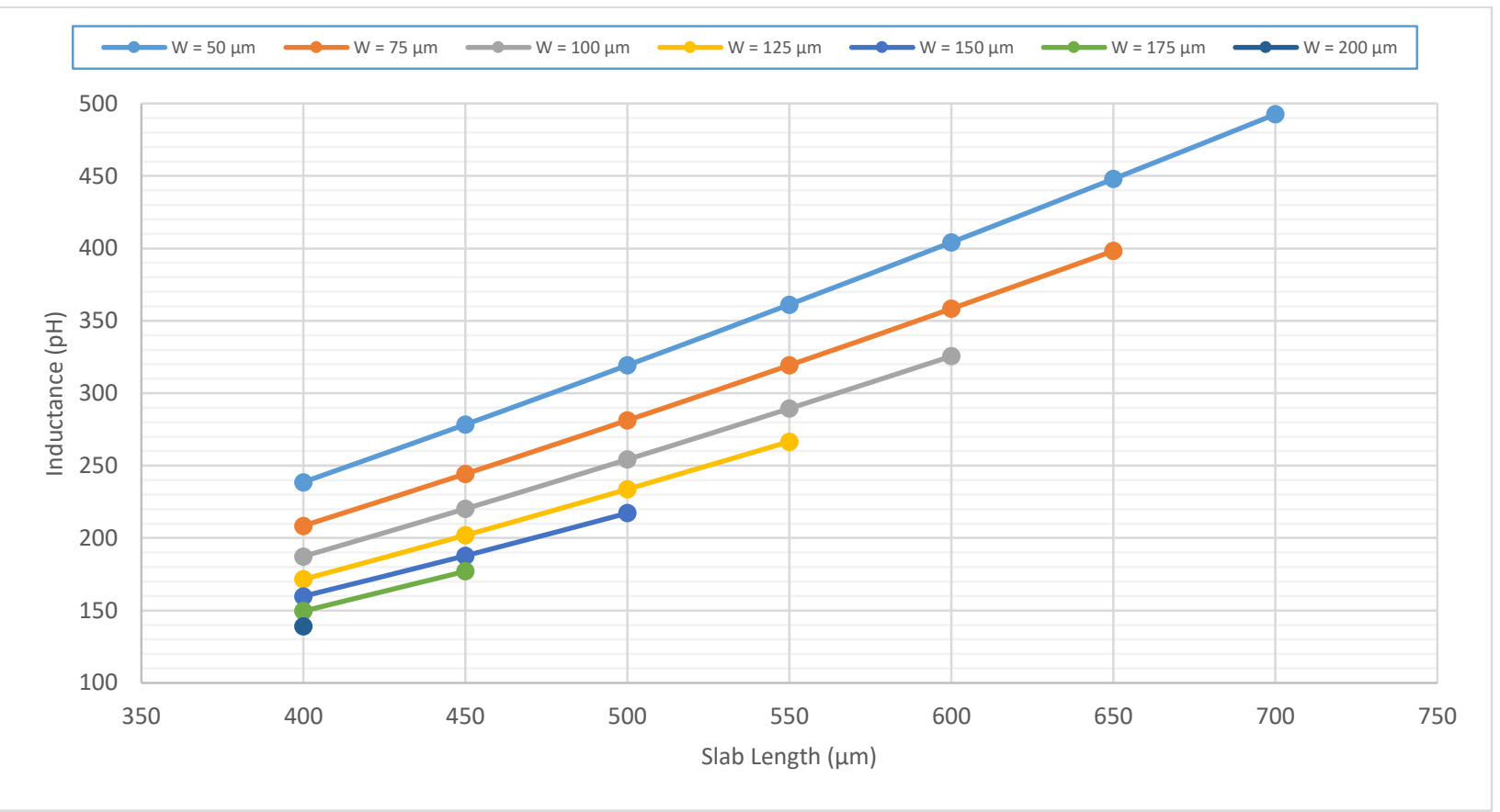

Figure 5.6: Slab inductance $(\mathrm{pH})$ as a function of length $(\mu \mathrm{m})$ for various widths $(\mu \mathrm{m})$. 
From Figure 5.6, it was observed that the inductance for a given slab length tends to decrease with increased slab width; this is consistent with the analysis from section 3.2 which determined that increased widths would reduce the characteristic impedance of the line and consequently the inductance per unit length.

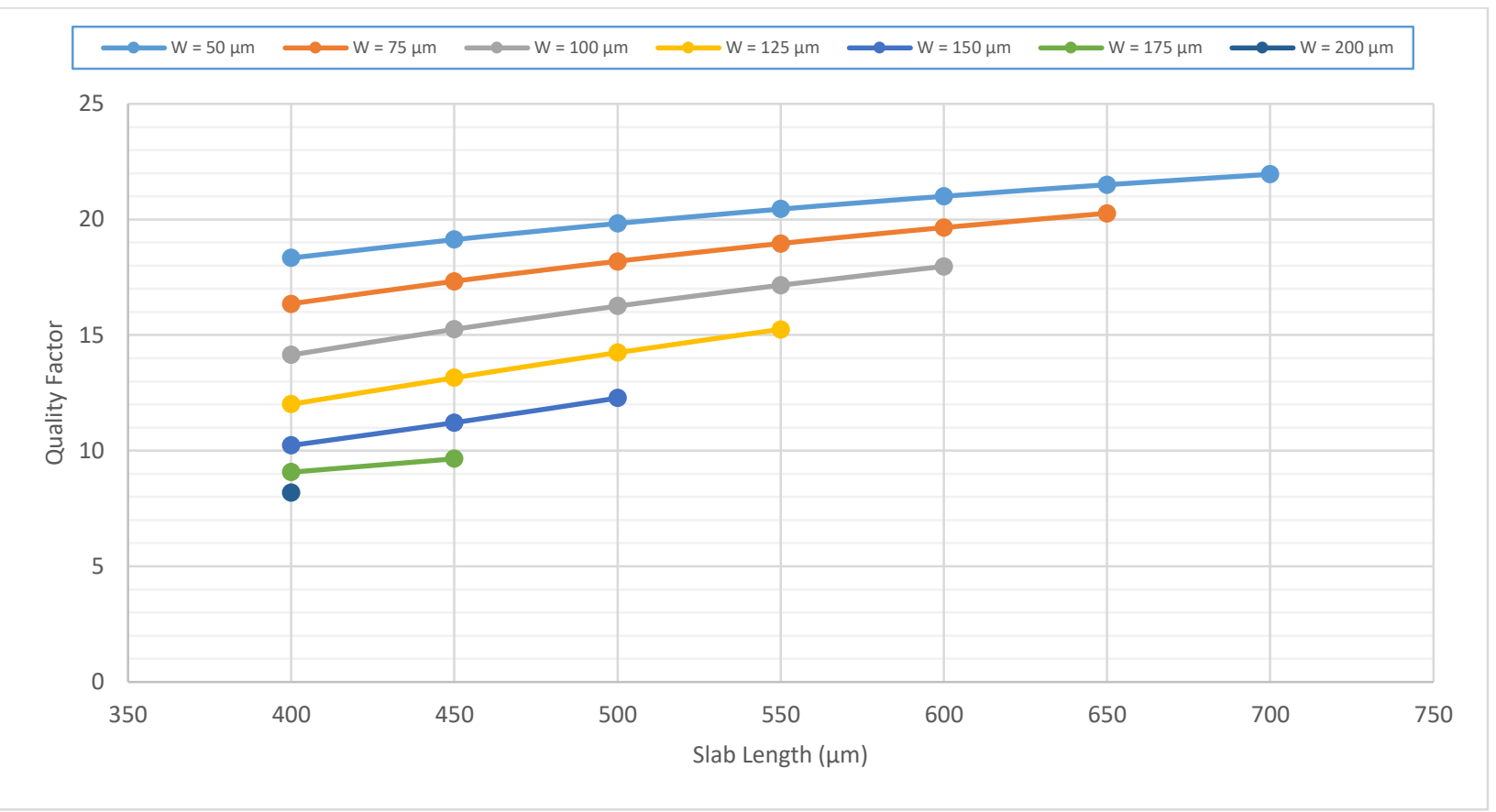

Figure 5.7: Quality Factor as a function of length $(\mu \mathrm{m})$ for various widths $(\mu \mathrm{m})$.

The quality factor was also observed to decrease with increased slab width (Figure 5.7) which suggests that the predicted decrease in series resistance is outpaced by the reduction in inductance per unit length.

Using the rough layout established in Figure 5.5, the initial slab length was set to $600 \mu \mathrm{m}$, leaving $100 \mu \mathrm{m} \times 100 \mu \mathrm{m}$ corners for the unit power cells. In order to maximize the quality factor, the initial slab width was set to $50 \mu \mathrm{m}$ for an estimated inductance of $404 \mathrm{pH}$ and quality factor of 21. In order to more accurately model the performance of the final layouts (including the center tap), the initial slab dimensions were re-simulated using an advanced EM simulation tool, EMX ${ }^{\circledR}$, developed by Integrand Software, Inc. The results are shown in Figure 5.8 below. 


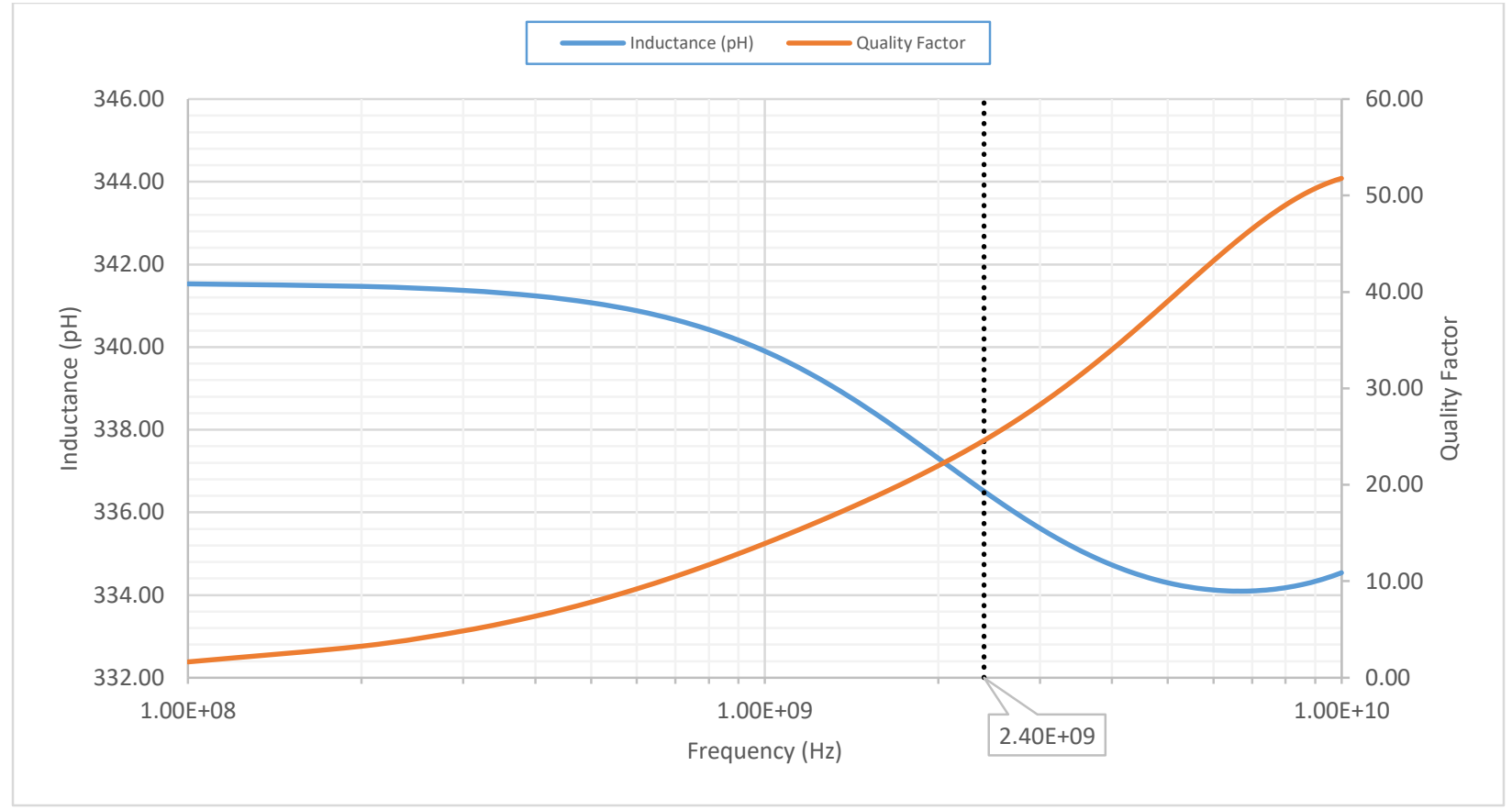

Figure 5.8: Advanced EM simulation of $600 \times 50 \mu \mathrm{m}$ slab inductor with center tap.

The advanced EM simulation revealed some degradation of the total inductance with the introduction of the center tap - down from $404 \mathrm{pH}$ to $337 \mathrm{pH}$ - however, quality factor increased from 21 to 25 . These initial values were used for the first iteration of the DAT design and implementation.

\subsubsection{Unit Power Cell Design}

The unit power cell consists of a power oscillator and associated injection circuitry and adopts the general topology discussed in Chapter 4. The conceptual design calls for four identical unit power cells that collectively achieve the system-level performance. Therefore, in order to design the unit cell, the system-level power and gain specifications given in Section 5.1 were translated to unit-level specifications.

\subsubsection{Determining unit cell specifications}

By symmetry, each unit power cell contributes $1 / 4$ the total output power, and therefore must be capable of delivering $125 \mathrm{~mW}$. It was assumed that there may be up to $3 \mathrm{~dB}$ of losses in the 
output matching network and so the unit cells were designed for an output power of $250 \mathrm{~mW}$. The $6 \mathrm{~dB}$ gain in output power from combining is offset by the $6 \mathrm{~dB}$ loss during input power distribution, however there is some expectation of losses in the input network. Therefore, the unit cell power gain was determined to be equal to the system-level gain specification plus an additional $3 \mathrm{~dB}$ of implementation margin.

Using the system output matching specification of $50 \Omega$, the overall required output peak voltage was determined as follows:

$$
P_{\text {out }}=\frac{\left(v_{p k}\right)^{2}}{2 \cdot R_{\text {load }, \text { sys }}} \therefore v_{p k}=\sqrt{2 \cdot P_{\text {out }} \cdot R_{\text {load }, \text { sys }}}=\sqrt{2 \cdot 1 \cdot 50}=10 \mathrm{~V}
$$

From (5.7), the overall required output current was determined:

$$
P_{\text {out }}=\frac{v_{p k} \cdot i_{p k}}{2} \therefore i_{p k}=\frac{2 \cdot P_{\text {out }}}{v_{p k}}=\frac{2 \cdot 1}{10}=0.2 \mathrm{~A}
$$

It was assumed that the output voltage dropped evenly across all four unit cells, yielding a unit output peak voltage of $2.5 \mathrm{~V}$ on the load side of the output transformers (Figure 5.9).

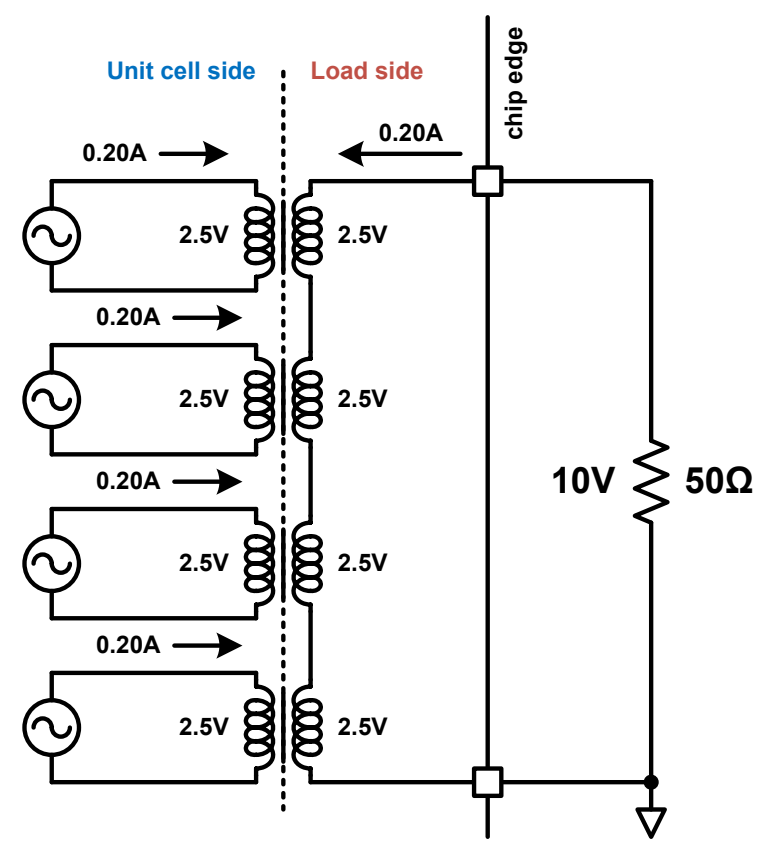

Figure 5.9: Assumed voltage and current values for output network. 
By Kirchhoff's current law, the component output current must therefore be equal to the overall output current of 0.2 A. Further assuming that the output voltage and current are phase aligned, the impedance on the load side of the output transformer was determined as follows:

$$
R_{\text {load }, \text { unit }}=\frac{v_{p k}}{i_{p k}}=\frac{2.5}{0.2}=12.5 \Omega
$$

Given the system specification for $V_{d d}=1.5 \mathrm{~V}$, the ideal maximum voltage swing on the unit cell side of the transformers was calculated as follows:

$$
\left.V_{d s, \text { max }}\right|_{\text {ideal }}=2 \cdot V_{d d}=2 \cdot 1.5=3 \mathrm{~V}
$$

Assuming a knee voltage of $0.4 \mathrm{~V}$ and $0.1 \mathrm{~V}$ of losses in the tank, the total output swing was assumed to be

$$
v_{\max }=2 \cdot V_{d d}-(0.4+0.1)=2.50 \mathrm{~V}
$$

Since the differential pair of the unit cell is a push-pull architecture, the maximum voltage swing is equal to the peak swing.

The ideal output transformer turns ratio was determined from the primary and secondary voltages using standard transformer equations as follows

$$
\frac{N_{p}}{N_{s}}=\frac{V_{p}}{V_{s}}=\frac{2.5}{2.5}=1.00
$$

From this turns ratio and the unit load, the equivalent tank resistance was determined

$$
R_{\text {tank }}=\left(\frac{N_{p}}{N_{s}}\right)^{2} \cdot R_{\text {load }, \text { unit }}=(1.00)^{2} \cdot 12.5=12.5 \Omega
$$

which, along with the maximum voltage swing, was used to determine the maximum current

$$
i_{\text {max }}=\frac{v_{\text {max }}}{R_{\text {tank }}}=\frac{2.5}{12.5}=0.20 \mathrm{~A}
$$




\subsubsection{Setting the oscillation frequency and determining the quality factors}

From section 5.3.1, the initial inductance was assumed to be $337 \mathrm{pH}$ with a quality factor of 25. Using this value, the required tank capacitance to achieve oscillation at the specified design frequency was calculated as follows

$$
\omega_{0}=\frac{1}{\sqrt{L C}} \therefore C=\frac{1}{L \cdot \omega_{0}^{2}}=\frac{1}{(337 p)(2 \pi \cdot 2.4 G)^{2}}=13.0 p F
$$

Assuming a quality factor of 40 for the tank capacitor, the effective parasitic resistance of the reactive components was estimated

$$
R_{\text {par }}=\frac{1}{G_{p a r}}=\frac{1}{\frac{1}{\omega_{0} L Q_{L}}+\frac{\omega_{0} C}{Q_{C}}}=\frac{1}{7.9 m+4.9 m}=78.2 \Omega
$$

The total tank resistance was further estimated by summing the parasitic and load resistances

$$
R_{\text {tot }}=\frac{1}{G_{\text {load }}+G_{\text {par }}}=\frac{1}{\frac{1}{R_{\text {load }}}+\frac{1}{R_{\text {par }}}}=\frac{1}{80.0 m+12.8 m}=10.8 \Omega
$$

Finally, the loaded and unloaded quality factors for the tank circuit were determined by considering the total and parasitic tank resistances respectively, as shown below

$$
\begin{gathered}
Q_{L}=R_{t o t} \cdot \sqrt{\frac{C}{L}}=10.8 \cdot \sqrt{\frac{130 p}{337 p}}=2.1 \\
Q_{U}=R_{p a r} \cdot \sqrt{\frac{C}{L}}=78.2 \cdot \sqrt{\frac{130 p}{337 p}}=15.4
\end{gathered}
$$

\subsubsection{Setting the locking range}

Having established the loaded quality factor for the resonator, the ratio between the injected current and resonating current required to meet the locking range specification could be determined. Re-arranging (equation from Chapter 4) as follows 


$$
2 \cdot \frac{Q_{L} \omega_{L}}{\omega_{0}}=\frac{I_{i n j}}{I_{o s c}} \cdot \frac{1}{\sqrt{1-\frac{I_{i n j}^{2}}{I_{o s c}^{2}}}}
$$

and substituting $\alpha=\frac{I_{\text {inj }}}{I_{\text {osc }}}$ and $\beta=\frac{Q_{L} \omega_{L}}{\omega_{0}}$, the equation was re-written and solved for $\alpha$

$$
2 \cdot \beta=\alpha \cdot \frac{1}{\sqrt{1-\alpha^{2}}} \therefore \alpha=\frac{2 \cdot \beta}{\sqrt{1+4 \beta^{2}}}=\frac{2 \cdot(0.442)}{\sqrt{1+4 \cdot(0.442)^{2}}}=0.662
$$

Next, the total current through the tank was determined by summing the required maximum current determined in (5.14) with the current dissipated through the parasitic resistance calculated in $(5.16)$

$$
i_{\text {out }}=i_{\text {max }}+\frac{v_{\text {max }}}{R_{\text {par }}}=0.20+\frac{2.50}{78.2}=232 \mathrm{~mA}
$$

Noting that the oscillator and injection currents are nominally phase aligned at the center frequency (and therefore the sum of their amplitudes at $f_{c}$ should equal the total current through the tank), the self-resonant current and injection current could be determined as follows

$$
i_{\text {out }}=i_{\text {osc }}+i_{\text {inj }}=i_{\text {osc }} \cdot(1+\alpha) \therefore i_{\text {osc }}=\frac{i_{\text {out }}}{1+\alpha}=\frac{0.232}{1+0.662}=140 \mathrm{~mA}
$$

and

$$
i_{\text {inj }}=i_{\text {osc }} \cdot \alpha=140 \mathrm{~m} \cdot 0.693=92.4 \mathrm{~mA}
$$

\subsubsection{Design of the unit power cell}

The schematic of the unit power cell is shown in Figure 5.10 below. 


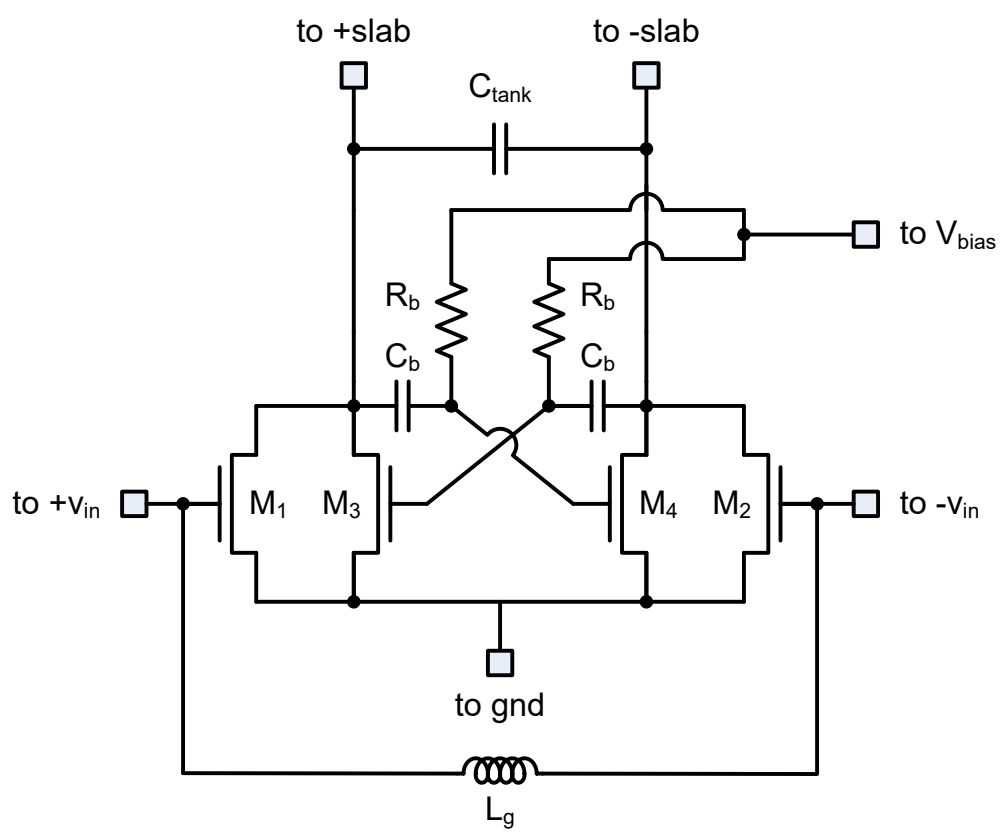

Figure 5.10: Unit power cell schematic.

With the tank capacitance set by (5.15), the next step was to size the oscillator core (consisting of transistors $\mathrm{M}_{3}$ and $\mathrm{M}_{4}$ ). Using the current specification from (5.24), the transistor width was adjusted for peak $\mathrm{f}_{\max }$ and the required transconductance (equal to one-half the effective load resistance with parastics). Next, RC high pass filters were attached to the gates of $\mathrm{M}_{3}$ and $\mathrm{M}_{4}$ to enable a dc bias voltage independent of the supply without blocking the RF signal. After verifying the self-oscillation frequency of the oscillator core, the injection transistors were sized to deliver the desired current at peak $\mathrm{f}_{\max }$. Finally, the gate inductor was sized to resonate out the gate capacitance and implemented as a slab inductor designed in a similar fashion to the tank slab inductors described in section 5.3.1.

\subsubsection{Unit Power Cell Layout}

The layout of the unit power cell was completed with three requirements in mind: first, the overall width of the active device area needed to less than the allocated width of the slab inductors; second, the plane of symmetry must enable the overall rotational symmetry of the DAT; and third, 
signals should be carried preferential in the low resistivity top metal layers. The unit power cell layout (without gate inductors) is shown in Figure 5.11 below.

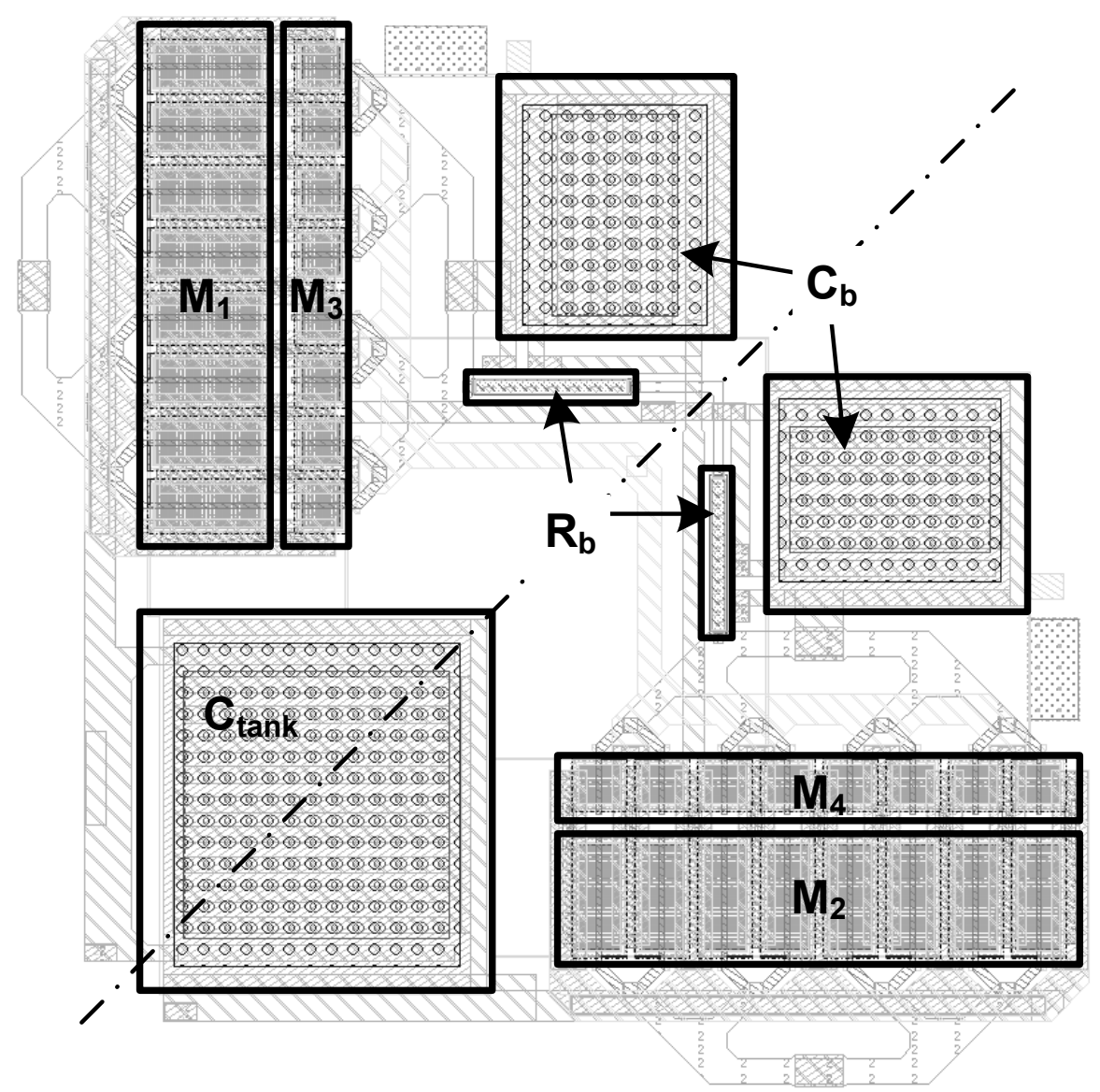

Figure 5.11: Unit power cell layout.

\subsubsection{Output Network Integration}

With the unit power cells completed, the slab inductor connections needed to be established and the magnetically coupled output ring sized. The minimum gap size for the top layer trace was $5 \mu \mathrm{m}$ and the objective optimization was to maximize the coupling factor to avoid significant ohmic losses in the internal winding. The capacitive coupling into the substrate needed to be minimized for the internal trace since the adjacency of the output terminals leads to a low substrate resistance between the output voltage and ground paired with a very large output voltage. The 
reference slab inductor from section 5.3.1 had been modified to $600 \times 80 \mu \mathrm{m}$ to accommodate the unit power cell layout. This was coupled with an equivalent length, $20 \mu \mathrm{m}$ wide line at $5 \mu \mathrm{m}$ and the combined structure (with center tap on the slab) was simulated using EMX. The resulting performance is provided in Figure 5.12 below.

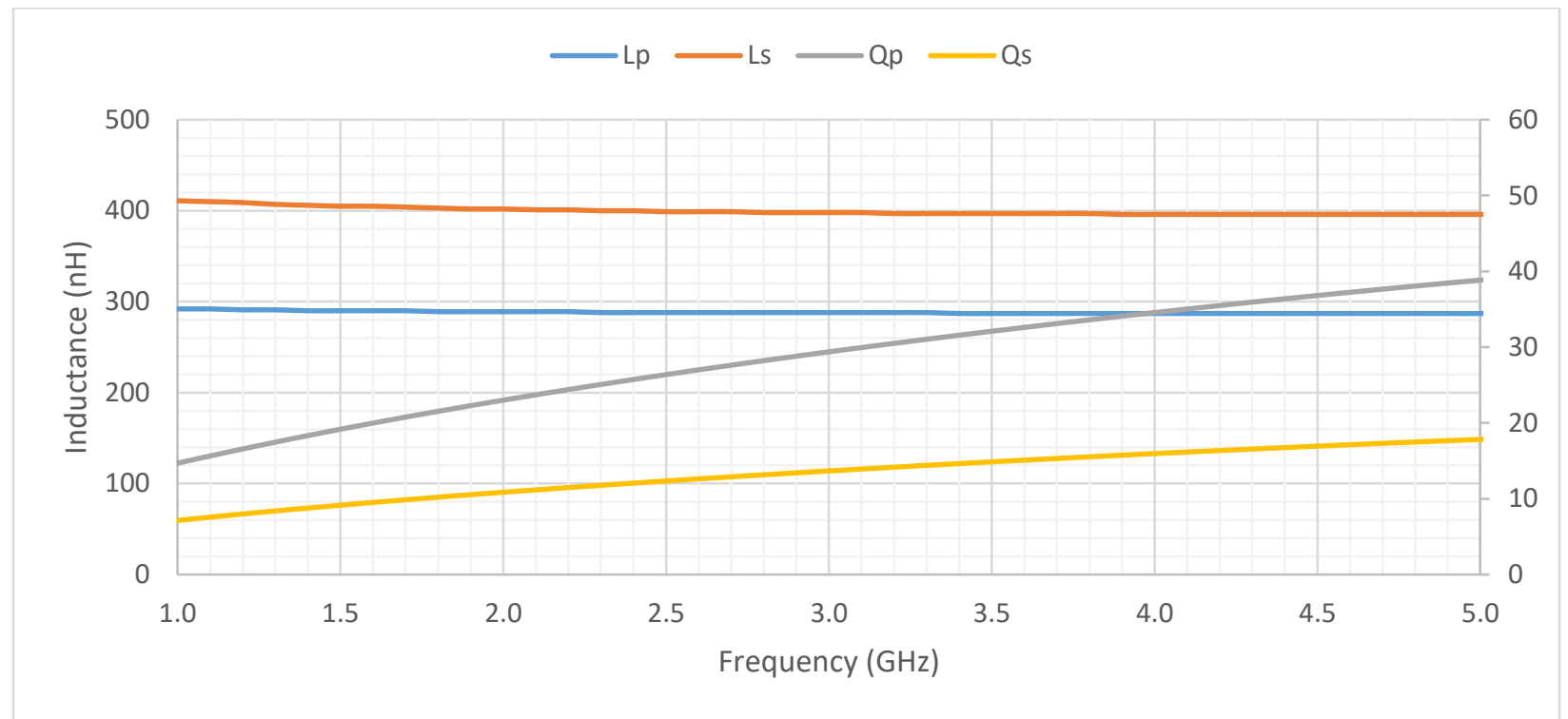

Figure 5.12: The primary and secondary inductances (blue and orange) and quality factors (grey and yellow) for a $600 \mu \mathrm{m}$ coupled line with $5 \mu \mathrm{m}$ spacing and $80 / 20 \mu \mathrm{m}$ widths.

The simulation results show the enhanced quality factor for the primary was retained although the inductance was diminished from the $600 \times 50 \mu \mathrm{m}$ slab. This was to be expected given the increase in slab width. The secondary quality factor was reasonable given the smaller line width. The coupling factor was determined to be 0.63 which aligns with Aoki's design estimates [12:134].

The coupled slabs were arranged in layout using the concept floorplan as a guide and extended to facilitate via connections to the drain terminals of the unit power cells. The $20 \mu \mathrm{m}$ traces were connected and adjusted to avoid overlap with the cross-connected capacitors. The final layout is shown in Figure 5.13 below. 


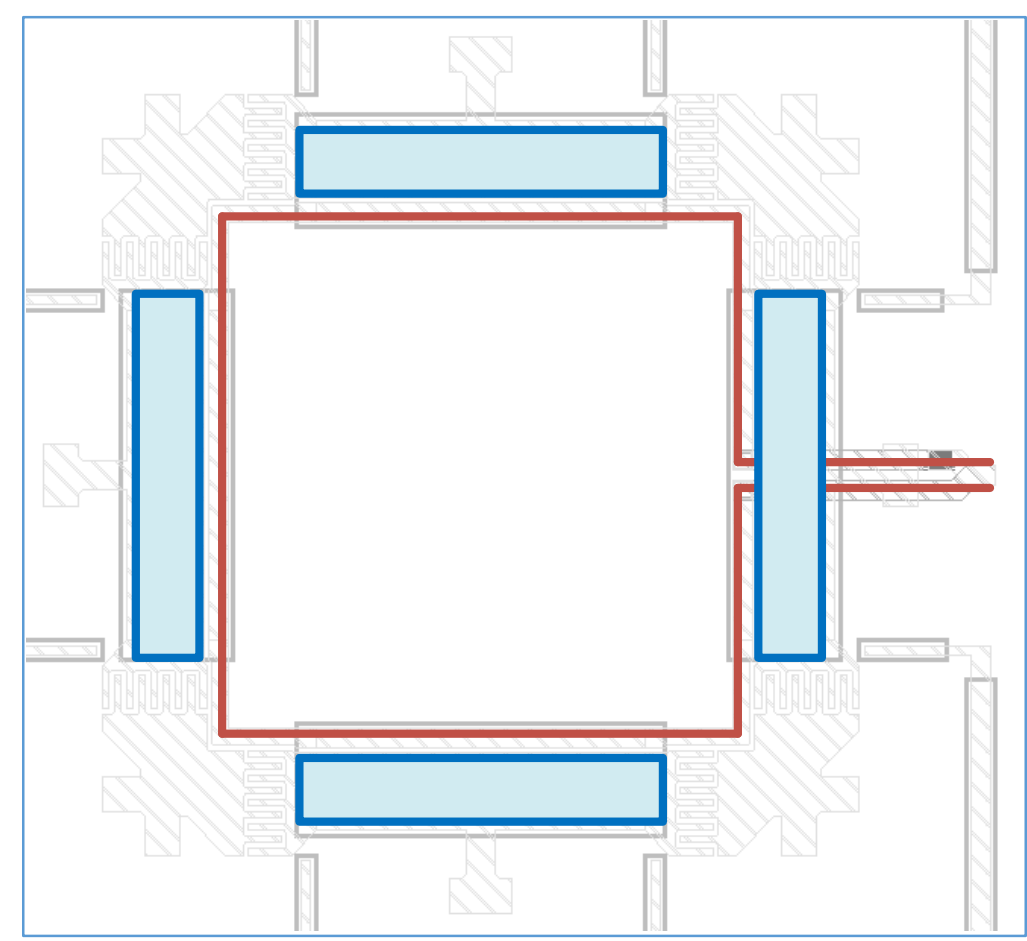

Figure 5.13: Output network with slab inductors and magnetic coupling ring.

The output trace was routed underneath the slab inductors to minimize disruptions to the slab geometry. The overall layout was optimized for rotational symmetry while maintaining sufficient space for the other modules.

The load pull was used to size the tuning capacitance at the output the inner winding. The results for the initial load pull are shown in Figure 5.14 below. 


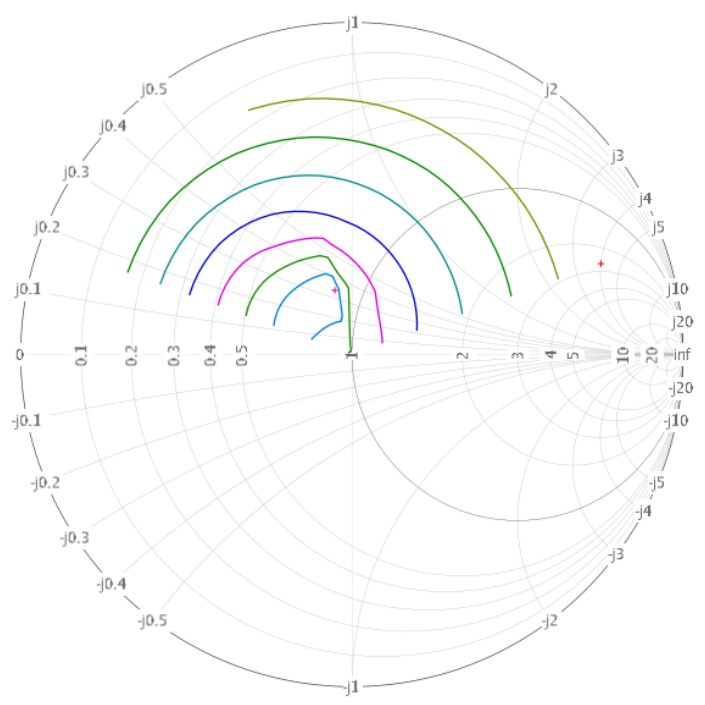

Figure 5.14: Simulated output power load pull for sizing output tuning capacitance.

The peak output power was already located reasonably close to the origin of the Smith chart and so it was determined that a small amount of capacitance would be required to bring the match to $50 \Omega$, however on implementation it was found that any additional capacitance across the output terminals degraded the performance and so the output tuning capacitance was eventually discarded.

\subsection{Input Matching Network Design \& Layout}

With the initial design of the DAT module complete, the input matching network module was designed to generate and distribute a differential input signal while optimally matching the 50 $\Omega$ input impedance for gain. This was accomplished by:

- first, designing the power dividers with the goal of minimizing phase variation and insertion loss; and,

- second, designing a transformer-based integrated balun to convert the differential impedance at the power dividers to a $50 \Omega$ single-ended impedance at the input to the PA. 


\subsubsection{Power Divider Design \& Layout}

The power divider takes the differential signals generated by the integrated balun and distributes them to the inputs of the unit power cells (Figure 5.2). Ensuring equal path lengths to avoid phase mismatches between power cells is essential due to the cross-connected nature of the system. If there are significant phase shifts between the power cells, the rotational symmetry will be broken and the adjacency assumptions that allowed the cross connecting of the tank capacitors and gate inductors will no longer hold.

In order to ensure equal phase shifts to each of the unit power cells, the differential signal is brought to the geometric center of the DAT and radiated out to the periphery. This is highlighted in Figure 5.15 below.

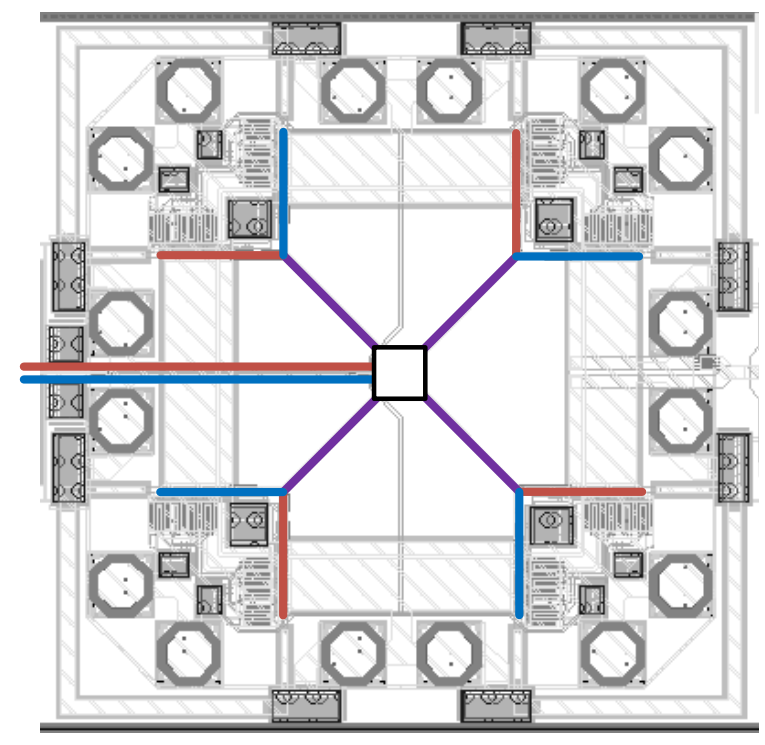

Figure 5.15: Power divider layout for symmetrical phase shifts.

The power dividers were simulated using EMX and the 5-port S-parameters were extracted. The insertion loss and phase shift relative to the input are shown in Figure 5.16 and Figure 5.17, respectively. It should be noted that because the desired symmetry was realized, all four curves 
appear to overlap perfectly for both figures. On closer inspection, small variations can be observed however these are unlikely to be significant.

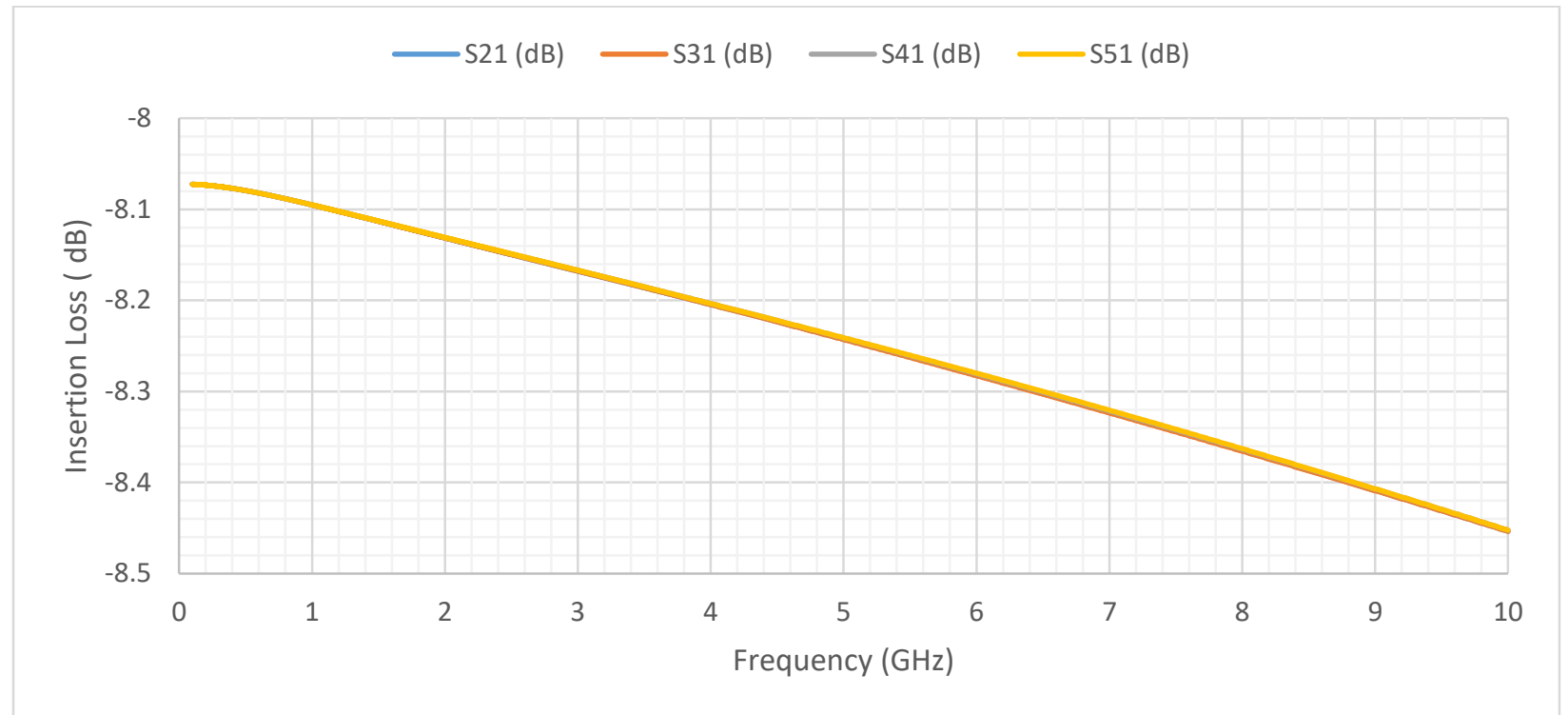

Figure 5.16: Power divider insertion loss over frequency.

An ideal lossless 4-way power divider will lead to a $6 \mathrm{~dB}$ different at the outputs relative to the input. Removing this desired contribution, the power divider contributes between 2.1 and $2.2 \mathrm{~dB}$ of loss over the operational frequency range (Figure 5.16).

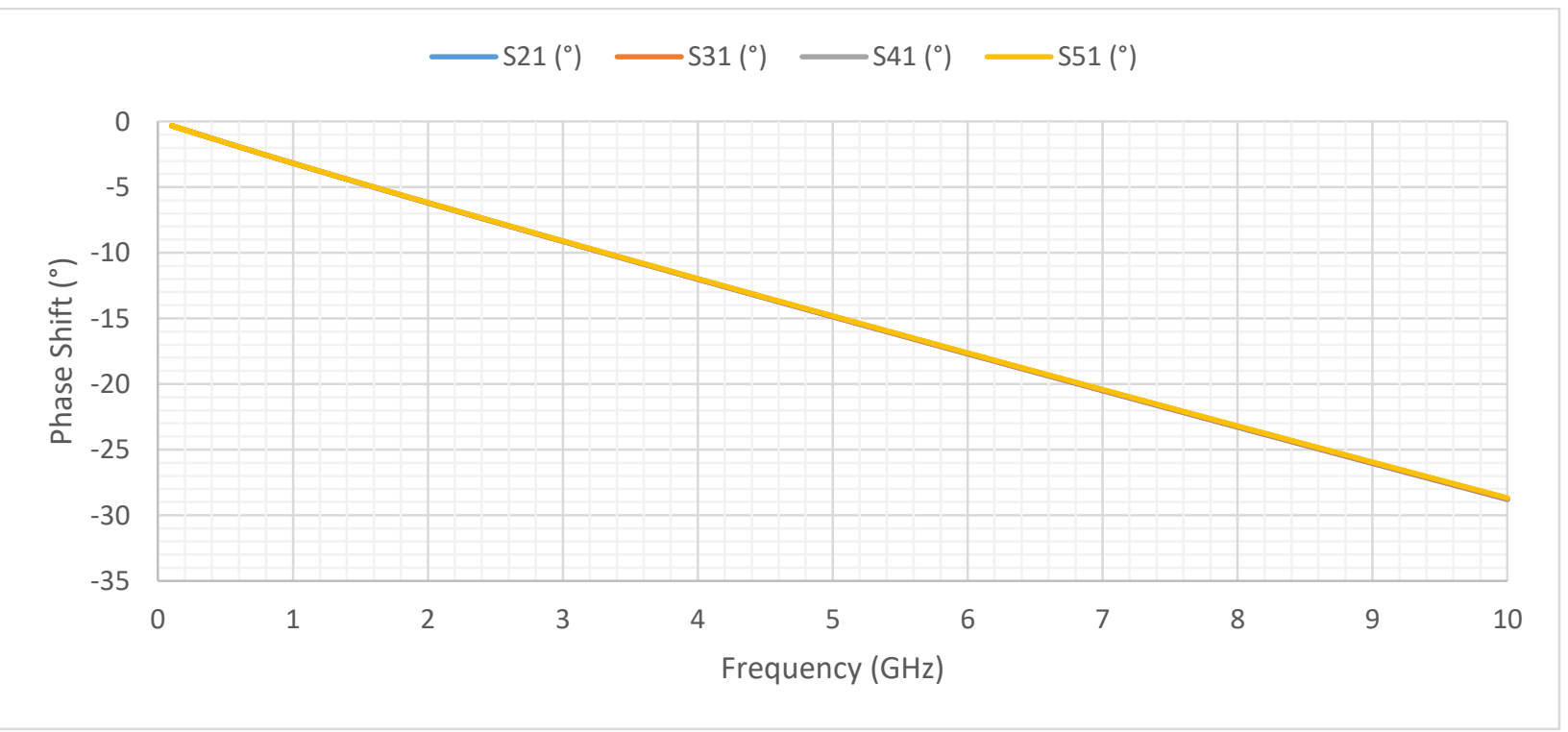

Figure 5.17: Power divider phase shift over frequency. 
The phase shift from the inputs is between 5 and 10 degrees over the operational frequency range. More importantly, this phase shift appears to be identical for all four paths.

\subsubsection{Integrated Balun Design \& Layout}

The integrated balun is based on the square symmetric center tapped transformer, first proposed by Rabjohn [18]. The symmetric layout solves the problem of the dislocation between the electrical and physical centers of asymmetric spiral inductor layouts while ensuring that the terminals are easily accessible at the outer edge of the transformer.

From section 5.2.1, the maximum outer diameter of the integrated balun was determined to be $350 \mu \mathrm{m}$. A symmetric differential kit inductor was generated and the layout flattened. The inductor spirals were severed along the horizontal axis of symmetry and re-organized to form a symmetric transformer. A center tap was introduced on the secondary coil and routed below the transformer coils to provide an access point for gate bias of the injection transistors. A trace for biasing the oscillator transistors was also routed beneath the transformer coils for symmetry. The design was iterated by simulation using EMX to maximize the $k$-factor. The final layout along with the equivalent electrical node map is shown in Figure 5.18 below.

a)

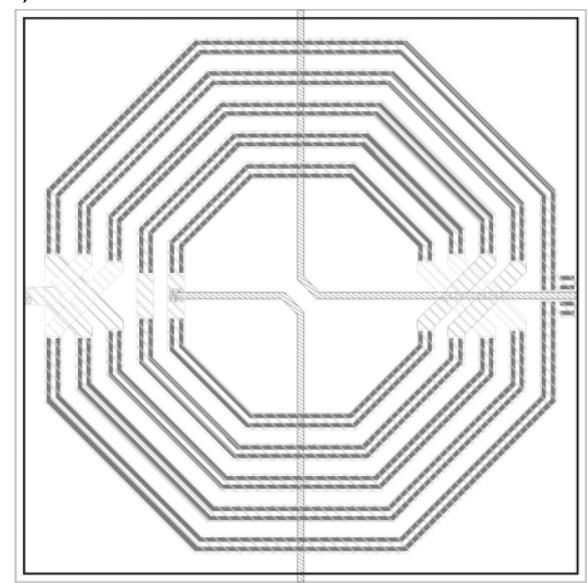

b)

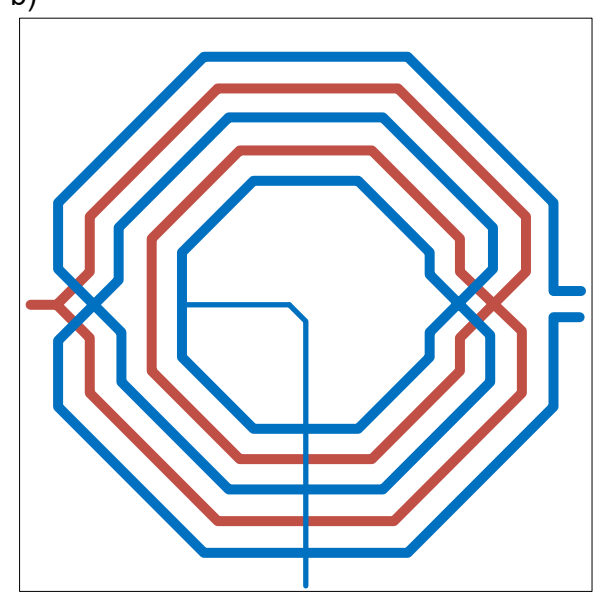

Figure 5.18: Integrated balun layout (left) and equivalent electric node map (right). 
From EMX, the coupling factor was determined to be 0.784 . The primary and secondary inductances and quality factors over frequency are shown in Figure 5.19 below.

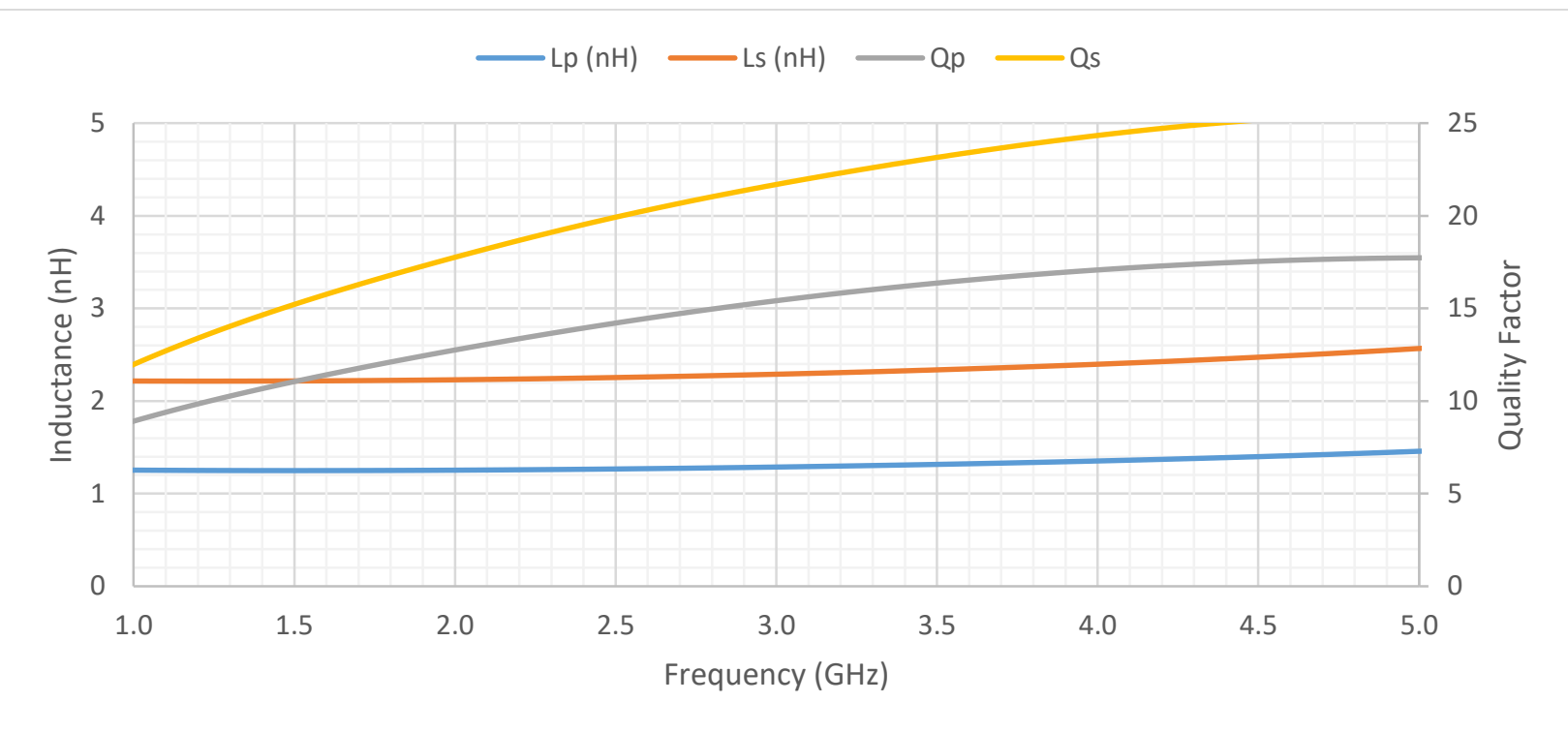

Figure 5.19: The primary and secondary inductances (blue and orange) and quality factors (grey and yellow) for the final integrated balun design.

As noted in section 2.5.2.2 on magnetic coupling, transformers require some tuning capacitances to resonate out the leakage inductances and minimize losses. Since the integrated balun is implemented using a center tapped transformer, these capacitances were included as shown in the schematic below.

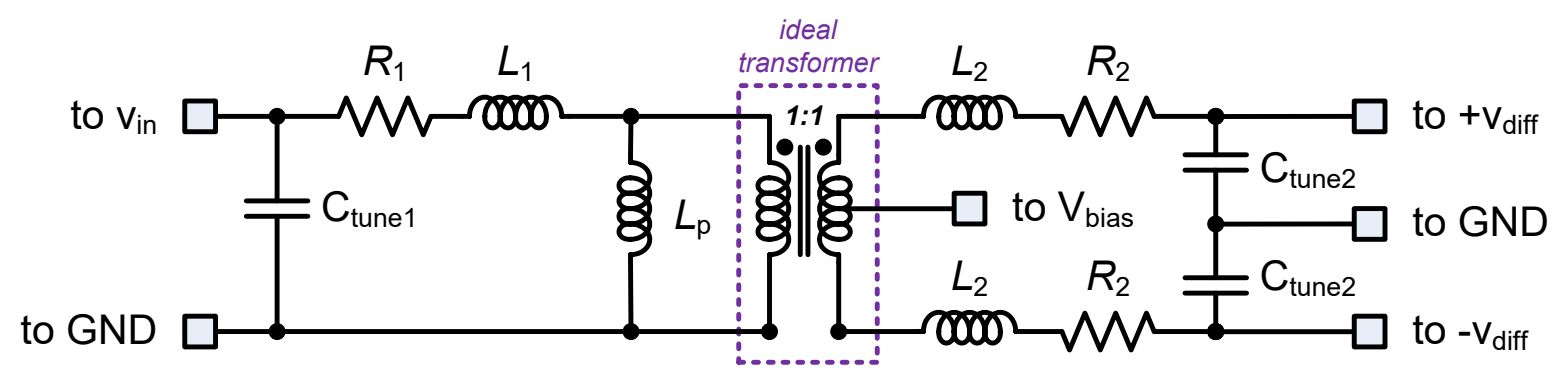

Figure 5.20: Integrated balun schematic model with center tap and tuning capacitors.

In order to size the capacitances, the integrated balun and power divider network were connected to the unit power cells. Next, the secondary tuning capacitors were swept until the input 
impedance measured at the primary of the integrated balun fell on the unit admittance circle. Finally, the primary tuning capacitor was swept until the system was matched to $50 \Omega$ at the center of the Smith chart. The tuning capacitors were implemented using dual MIM capacitors and their layout locations are highlighted in Figure 5.21 below.

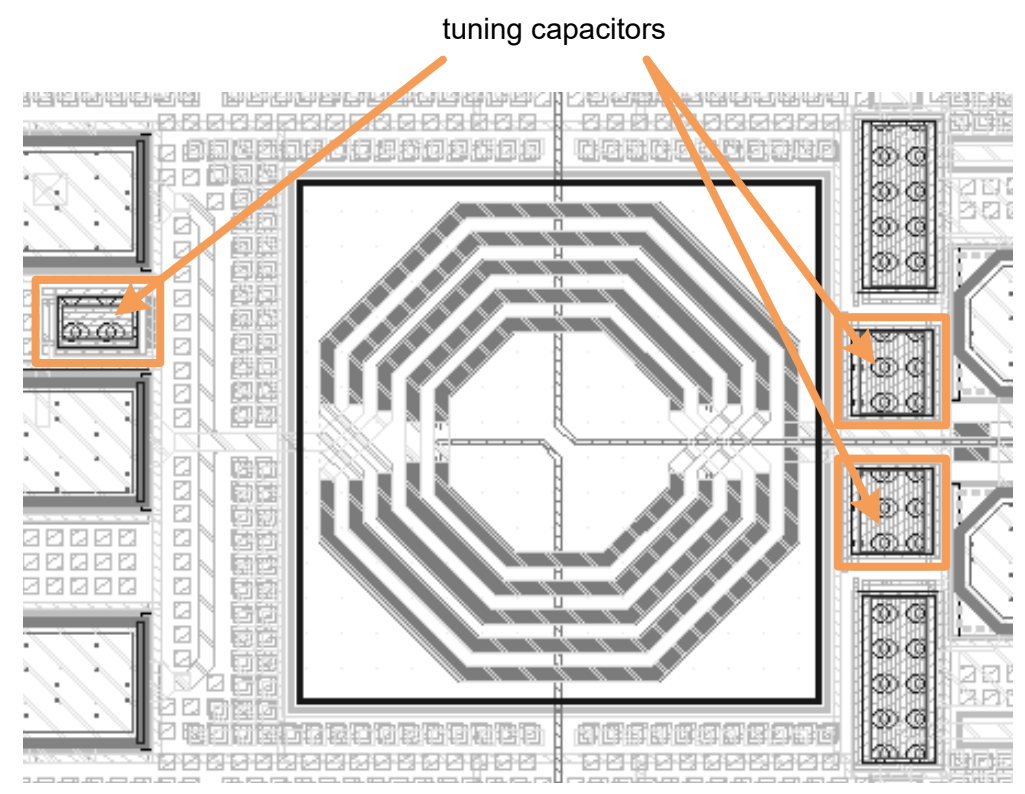

Figure 5.21: Integrated balun layout showing locations of tuning capacitors.

\subsection{Integration and Top-Level Layout}

With the component module designs completed and their reference layouts generated, the module integration and top-level layout was finalized. First, the module layouts were organized according to the concept floor plan from section 5.2.1. The respective node connections were established using the top three metal layers to minimize losses. The bias points were connected to $85 \mu \mathrm{m}$ octagonal bond pads and the $150 \mu \mathrm{m}$ pitch input and output GSG bond pads were placed along the midline at the left and right chip edges. Despite the virtual ac grounds precluding the requirement for on-chip bypass capacitors at the supply pads, space was available and they were included to mitigate any phase mismatches that might occur. Finally, the voltage bias networks 
for the oscillator and injection transistors were connected to $85 \mu \mathrm{m}$ octagonal bond pads above and below the integrated balun; spaced away from the DAT to simplify the bond wire connections. The final layout with annotations is shown in Figure 5.22 below.

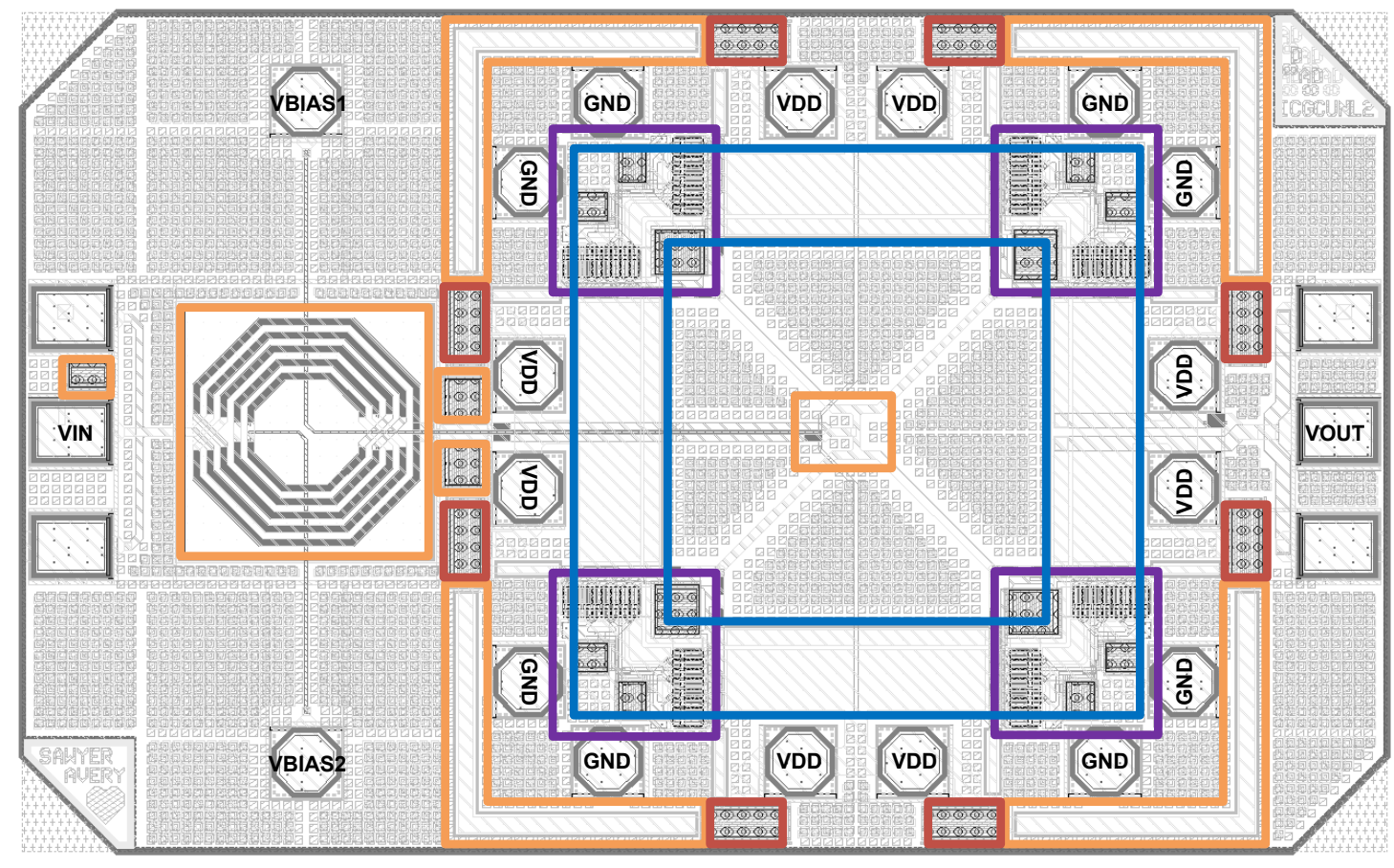

Figure 5.22: Fully integrated layout with annotations.

The orange highlighted regions correspond to the input network components and modules, and the purple highlighted regions correspond to unit power cells. The blue highlighted region is the DAT structure, and the red highlighted regions are the bypass capacitors. The bond pads are labeled with the appropriate bias voltage names and the input and output GSG bond pads are labelled accordingly.

\subsection{Final Implementation}

The nature of the design - using complex bespoke passive component layouts - required a tight iteration cycle between design and implementation. The design went through several 
iterations before converging to the final fabrication version. Tables of the final parameter values and reference design kit models are provided below, organized by active and passive components.

Table 5.2: Active Component Summary - Final Design

\begin{tabular}{ccccl}
\hline Ser & Parameter & $\mathbf{M}_{\mathbf{1}} / \mathbf{M}_{\mathbf{2}}$ & $\mathbf{M}_{\mathbf{3}} / \mathbf{M}_{\mathbf{4}}$ & Units \\
\hline \hline 1 & Component Name & nfet33_rf & nfet33_rf & - \\
2 & $M$ & 8 & 8 & $\mathrm{GHz}$ \\
3 & $L_{\text {gate }}$ & 400 & 400 & $\mathrm{~nm}$ \\
4 & $N$ & 24 & 8 & fingers \\
4 & $W_{\text {finger }}$ & 8 & 8 & $\mu \mathrm{m}$ \\
\hline
\end{tabular}

Table 5.3: Passive Component Summary - Final Design

\begin{tabular}{|c|c|c|c|c|c|c|c|}
\hline Ser & Parameter & $\mathrm{C}_{\text {tune1 }}$ & $\mathrm{C}_{\text {tune2 }}$ & $\mathbf{C}_{\text {bias }}$ & $\mathrm{C}_{\text {tank }}$ & $R_{\text {bias }}$ & Units \\
\hline 1 & Component & dualmimcap & dualmimcap & dualmimcap & dualmimcap & oprppres & - \\
\hline 2 & $W$ & 20 & 40 & 25 & 53 & 2.5 & $\mu \mathrm{m}$ \\
\hline 3 & $L$ & 45 & 50 & 40 & 53 & 30 & $\mu \mathrm{m}$ \\
\hline 3 & $R$ & - & - & - & - & 2.8 & $\mathrm{k} \Omega$ \\
\hline 4 & C & 5.05 & 9.86 & 5.39 & 13.37 & - & $\mathrm{pH}$ \\
\hline
\end{tabular}

The final implementation was connected to a virtual test bench and simulated to evaluate performance. Small signal analysis was completed using an S-parameters simulation. A harmonic balance simulation was used to extract the performance over a range of input powers; the results are shown in the following figures.

From the S-parameter simulation, relevant performance information is drawn from the return loss $\left(\mathrm{S}_{11}\right)$ and forward gain $\left(\mathrm{S}_{21}\right)$, shown here in Figure 5.23 below. 


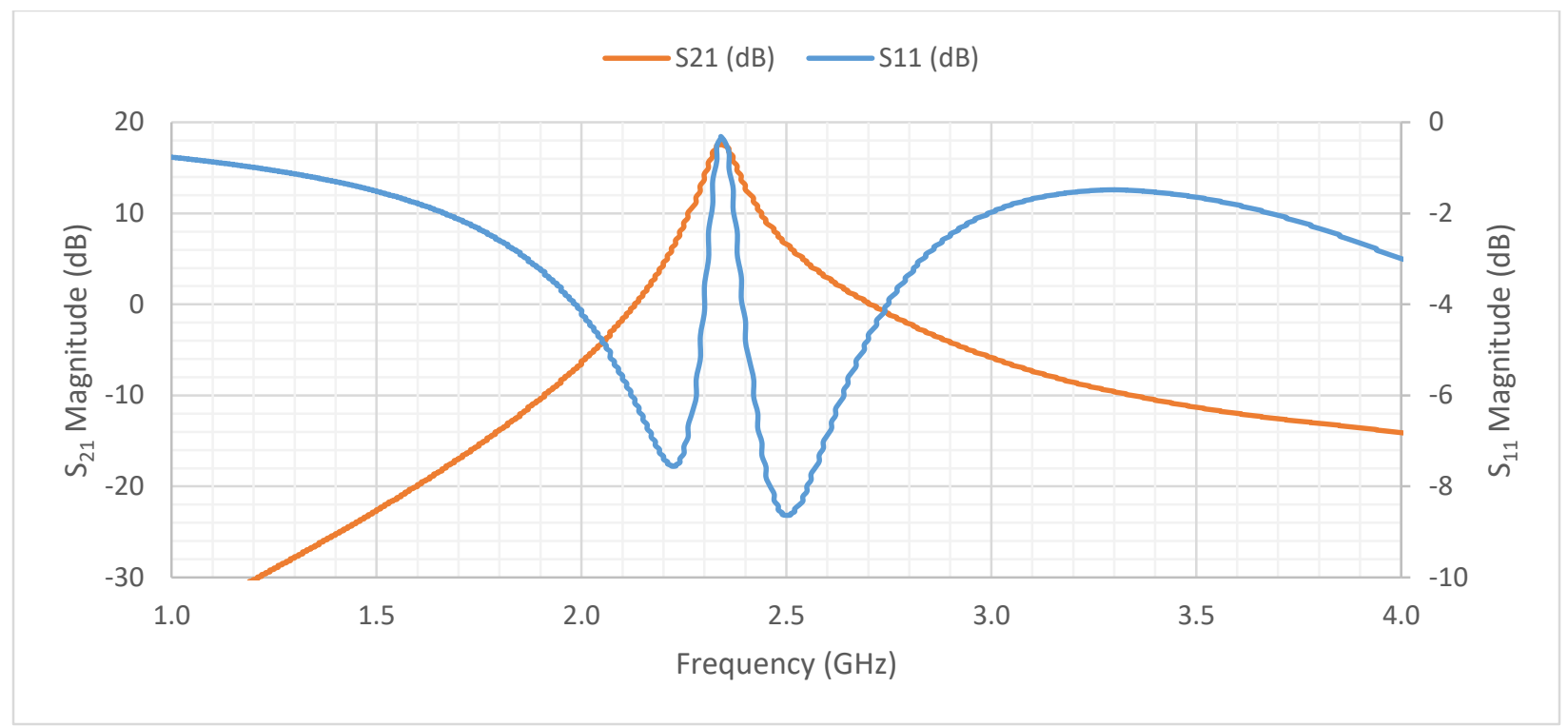

Figure 5.23: Simulated $S_{11}$ (blue) and $S_{21}$ (orange) at over frequency.

The forward gain curve indicates very narrow band performance with the peak located just below the design frequency of $2.4 \mathrm{GHz}$. The return loss has an odd resonance that corresponds to the forward gain peak and is believed to be associated with the instability caused by the oscillator. The interpolated return loss falls below $10 \mathrm{~dB}$ in the desired frequency range which is reasonable for a narrow band match.

From the harmonic balance simulation, relevant information is extracted from the output power and gain, shown in Figure 5.24 below. 


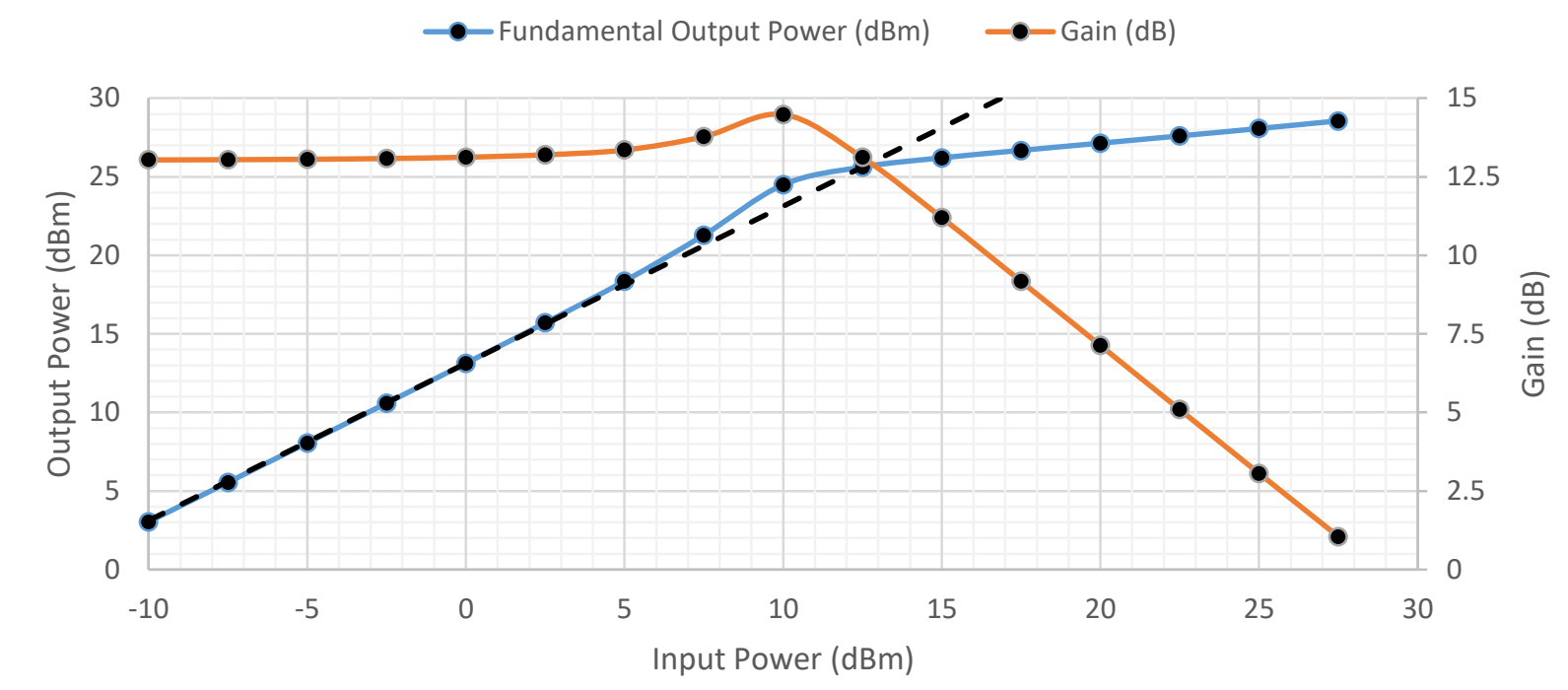

Figure 5.24: Simulated output power (blue) and gain (orange) at $2.4 \mathrm{GHz}$.

The simulated output power begins to compress at an input power of $15 \mathrm{dBm}$, with the output $\mathrm{P}_{1 \mathrm{~dB}}$ equal to $25.94 \mathrm{dBm}$. The maximum gain is $14.5 \mathrm{~dB}$ and it occurs at $10 \mathrm{dBm}$ input power. The power added efficiency is low for input powers below $5 \mathrm{dBm}$ and increases to a maximum of $23 \%$ at an input power of $20 \mathrm{dBm}$.

\subsection{Chapter Summary}

In this chapter, the design methodology for an injection-locked DAT was reviewed and the implementation and layout for $500 \mathrm{~mW}$ amplifier at 2.4 GHz was presented. Due to the geometry requirements imposed by the available die area, the methodology started by pre-supposing layout constraints and sizing the large slab inductances prior to designing the unit power cells. The general amplifier specification was reduced to a unit cell specification that drove the active device sizing. The input network was then designed to match the input to a $50 \Omega$ system impedance. The large and complex passive structures led to a tight iteration cycle of design implementation and layout that converged on a final optimized design. This final design was fabricated and tested to 
verify performance. The measurement results and analysis for the fabricated chip are presented in the next chapter. 


\section{Chapter 6: Results \& Analysis}

This chapter presents the measurement results and analysis. It begins with an overview of the test apparatus followed by a presentation of the test measurements. It concludes with an analysis of the results and discusses potential explanations for the observed performance.

\subsection{Test Apparatus}

The initial testing was completed at the Carleton University Department of Electronics. The test apparatus consisted of the following:

- a printed circuit board (PCB), DC harness and power supplies for biasing;

- a multi-meter with needle probes for verifying bias voltages in close proximity to the chip;

- a probe station equipped with pair of ground-signal-ground (GSG) probes and associated coaxial cables to provide the RF stimuli;

- a vector network analyzer (VNA) for small signal analysis; and,

- a fixed attenuator, signal generator and spectrum analyzer for large signal analysis.

First, the design and layout of the measurement board will be provided, followed by a review of the test bench configurations for both small signal and large signal analysis.

\subsubsection{PCB Design \& Layout}

Although the RF input and output ports were designed to be accessed through probes, the DC biasing was intended to be delivered through bond wire connections. This required the design and fabrication of a simple printed circuit board (PCB) for measurement. In order to maintain the rotational symmetry of the DAT, the chip itself has several pads for connecting the supply and ground nodes; these were consolidated off chip to common bias nodes. Due to the simplicity of the connections, all the traces were implemented in the top layer of the PCB. This avoided the use 
of through holes which would otherwise interfere with the vacuum hold down stages of the bonding machine and probe station. The layout of the board was iterated with a view to maximizing flexibility when bonding; ultimately leading to a large, thermally isolated landing area that doubles as the ground plane bond pad. The final schematic and layout are shown below (Figure 6.1) with the fabricated board measuring 4 x $4 \mathrm{~cm}$.
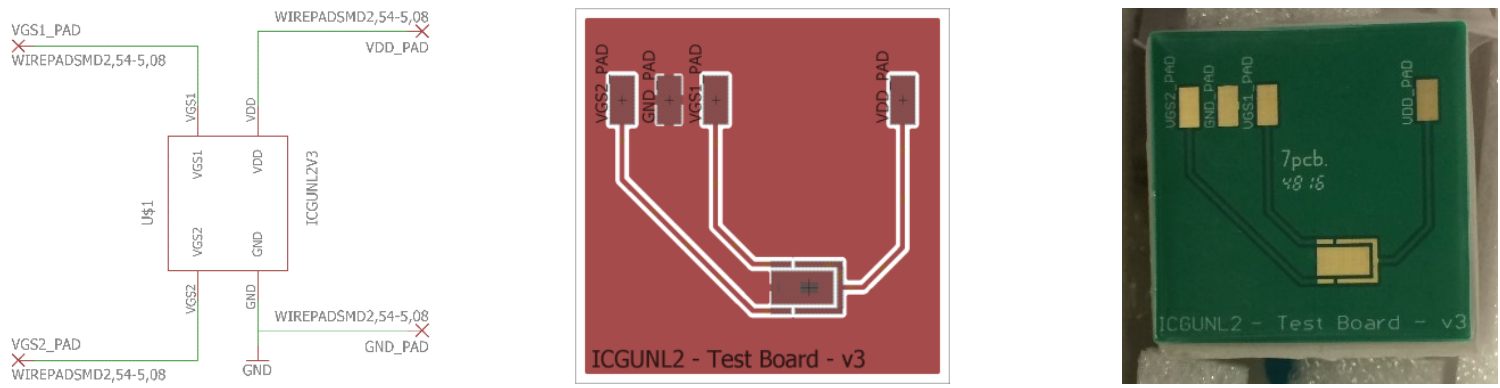

Figure 6.1: Schematic (left) and layout (middle) for the fabricated (right) measurement board. ${ }^{31}$

\subsubsection{Measurement Test Bench}

Chips were mounted to the measurement boards and bond wire connections made to the appropriate DC nodes. A micrograph showing the position of the bond wires is shown in Figure 6.2 below.

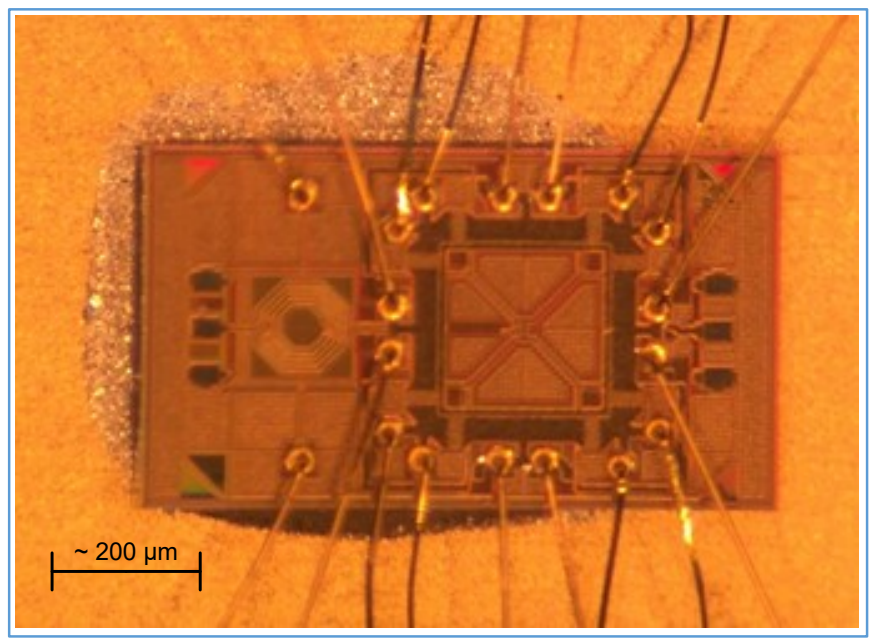

Figure 6.2: Micrograph of the mounted chip with bond wire connections.

\footnotetext{
31 The printed circuit board was designed using the EAGLE software package from Autodesk.
} 
The angle and spacing of the bond wires were adjusted to ensure that they did interfere with landing the RF probes at the input and output pads located at the left and right. A wire harness was surface soldered to the DC pads on the measurement board and the board was fixed to the stage of the probing station using the vacuum hold down system (Figure 6.3).

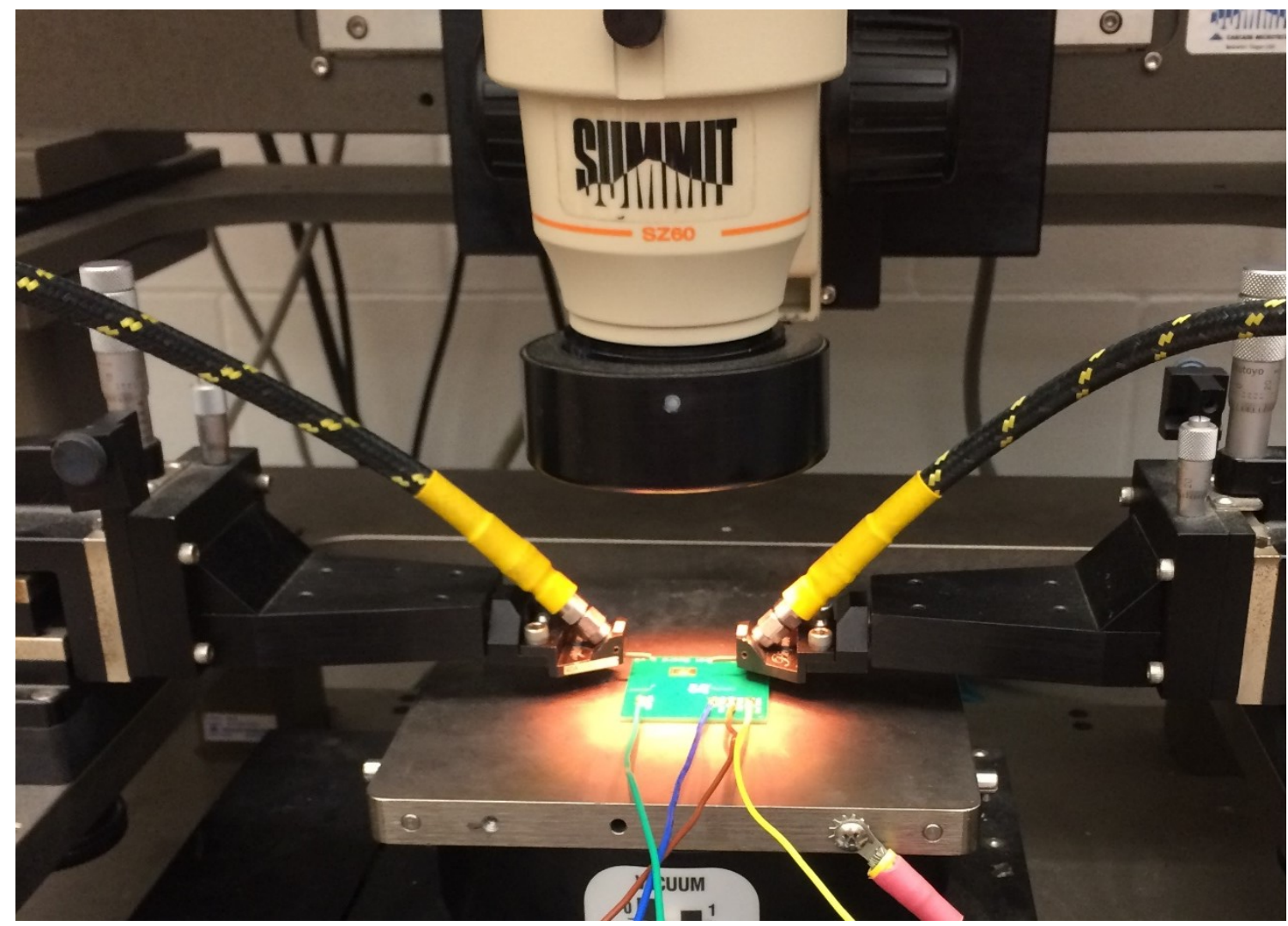

Figure 6.3: Photograph of the measurement board positioned in the probe station.

\subsubsection{Small and large signal test set-up}

The measurements were performed using two test bench configurations: a small signal analysis setup using a vector network analyzer (Figure 6.4a) and a large signal analysis setup using a signal generator and spectrum analyzer (Figure 6.4b). 
a)

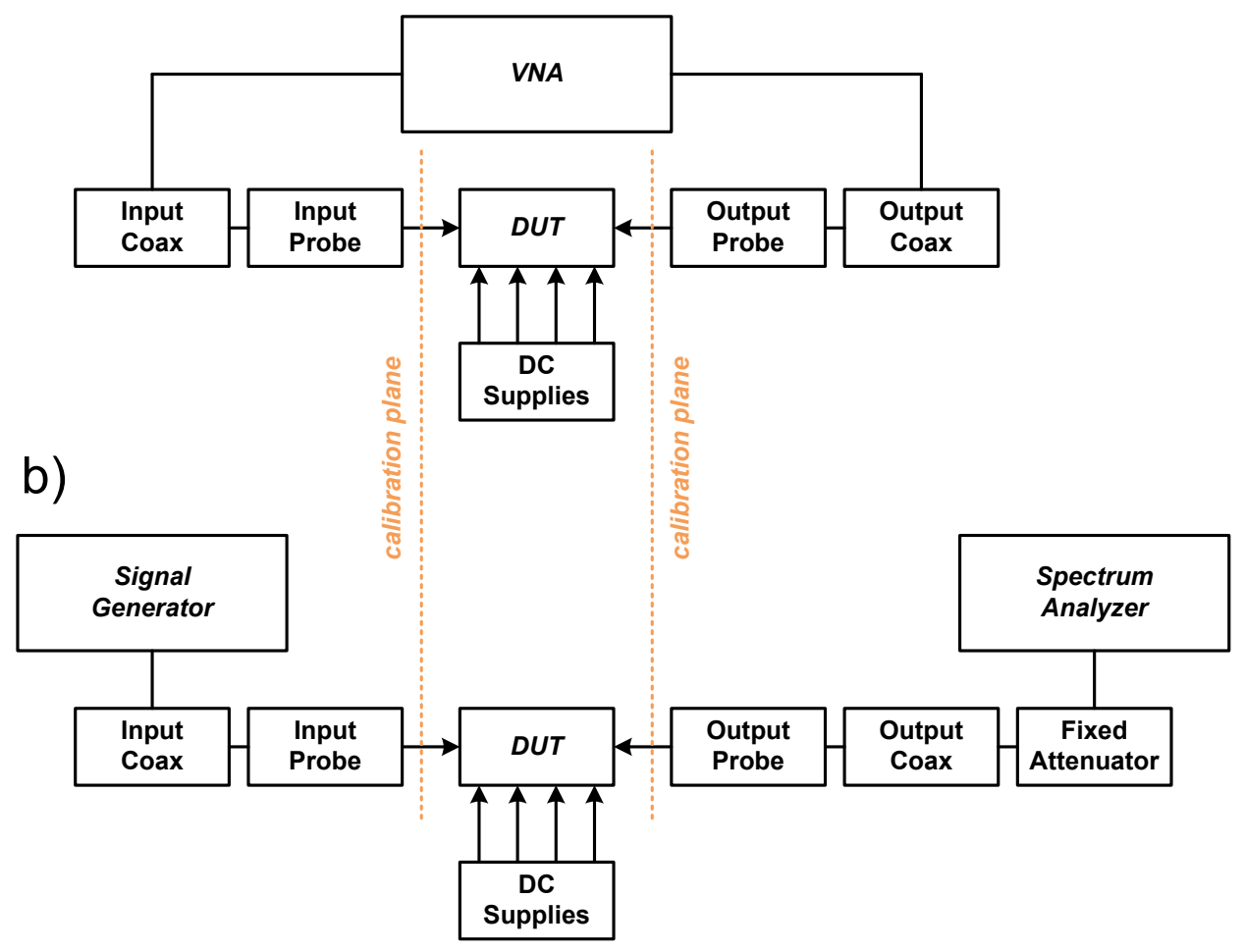

Figure 6.4: Block diagrams for the small signal (a) and large signal (b) test benches.

For small signal analysis, the calibration plane was brought to the probe tips using a calibration substrate in order to de-embed the effects of the probes and cables. For large signal analysis, the coaxial cable losses were measured directly and the 50-ohm transmission line on the calibration substrate was used to estimate the losses contributed by the probes. In order to protect the input of the spectrum analyzer, a fixed attenuation of $20 \mathrm{~dB}$ was inserted into the output path for large signal analysis only.

\subsection{Measured Results}

The test plan for the amplifier was conducted in three stages for three chips from the batch. First, the operating point was verified by setting the dc bias voltages and measuring the dc current draw. Second, the vector network analyzer was used to extract the small signal S-parameters. 
Finally, the large signal operation of the amplifier was measured using the signal generator and spectrum analyzer. As will become clear in the following sections, significant performance discrepancies between the measured and simulated results ultimately led to an alternative test strategy gear towards determining the potential root causes of the degradation as opposed to fully characterizing the amplifier performance.

\subsubsection{Operating Point Verification}

The first phase of measurement involved the verification of the DC bias points to determine if the active devices in the unit power cells were functional. The bias voltages were referenced to the voltages at the solder pads on the measurement board in order to remove harness losses.

First, the supply voltage was set to $1.5 \mathrm{~V}$ and then the oscillator gate bias voltage was set to $1.5 \mathrm{~V}$. Next, the injection gate bias voltage was swept until the supply current reached the design point of $1.25 \mathrm{~A}$. The required gate bias was $0.85 \mathrm{~V}$, approximately $0.1 \mathrm{~V}$ higher than in simulation.

\subsubsection{Small Signal Measurements}

Small signal measurements were conducted using a vector network analyzer. The frequency was swept from 1 to $5 \mathrm{GHz}$ at an input power of $10 \mathrm{dBm}$. The resulting return loss $\left(\mathrm{S}_{11}\right)$ and forward gain $\left(\mathrm{S}_{21}\right)$ are provided in Figure 6.5 below. 


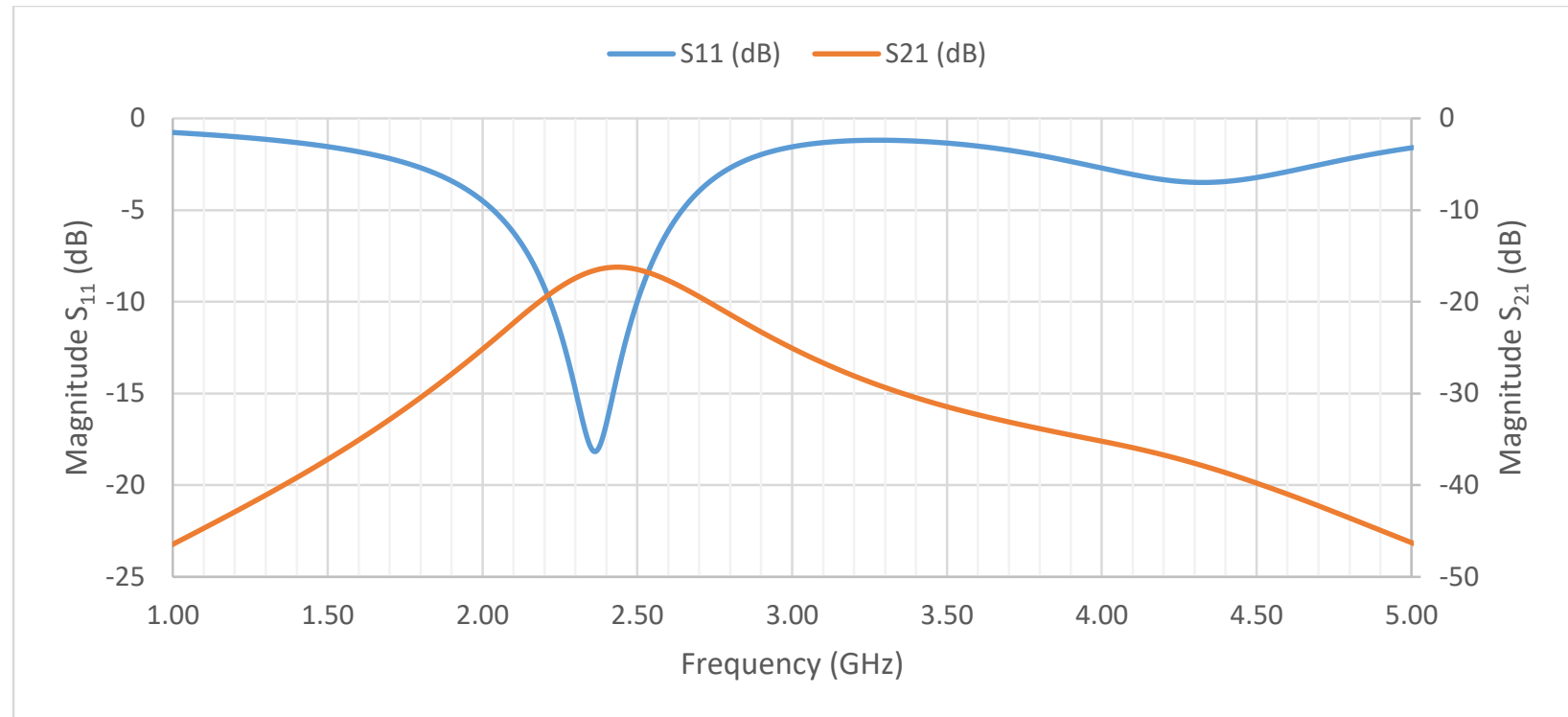

Figure 6.5: $\mathrm{S}_{11}$ (blue) and $\mathrm{S}_{21}$ (orange) measured over frequency at $\mathrm{P}_{\mathrm{in}}=10 \mathrm{dBm}$.

The return loss achieves a minimum of $-18 \mathrm{~dB}$ at $2.36 \mathrm{GHz}$ and the forward gain achieves a maximum of $-16 \mathrm{~dB}$ at $2.43 \mathrm{GHz}$. The shapes of the $\mathrm{S}_{11}$ and $\mathrm{S}_{21}$ curves correspond to the overall shapes of the simulated results; however, the amplitude of the $\mathrm{S}_{21}$ curve is noticeably and dramatically reduced.

\subsubsection{Large Signal Measurements}

Large signal measurements were conducted using a signal generator and spectrum analyzer. The input power was set to $10 \mathrm{dBm}$ and the frequency swept from 1 to $4 \mathrm{GHz}$. The resulting output power is shown in Figure 6.6 below. 


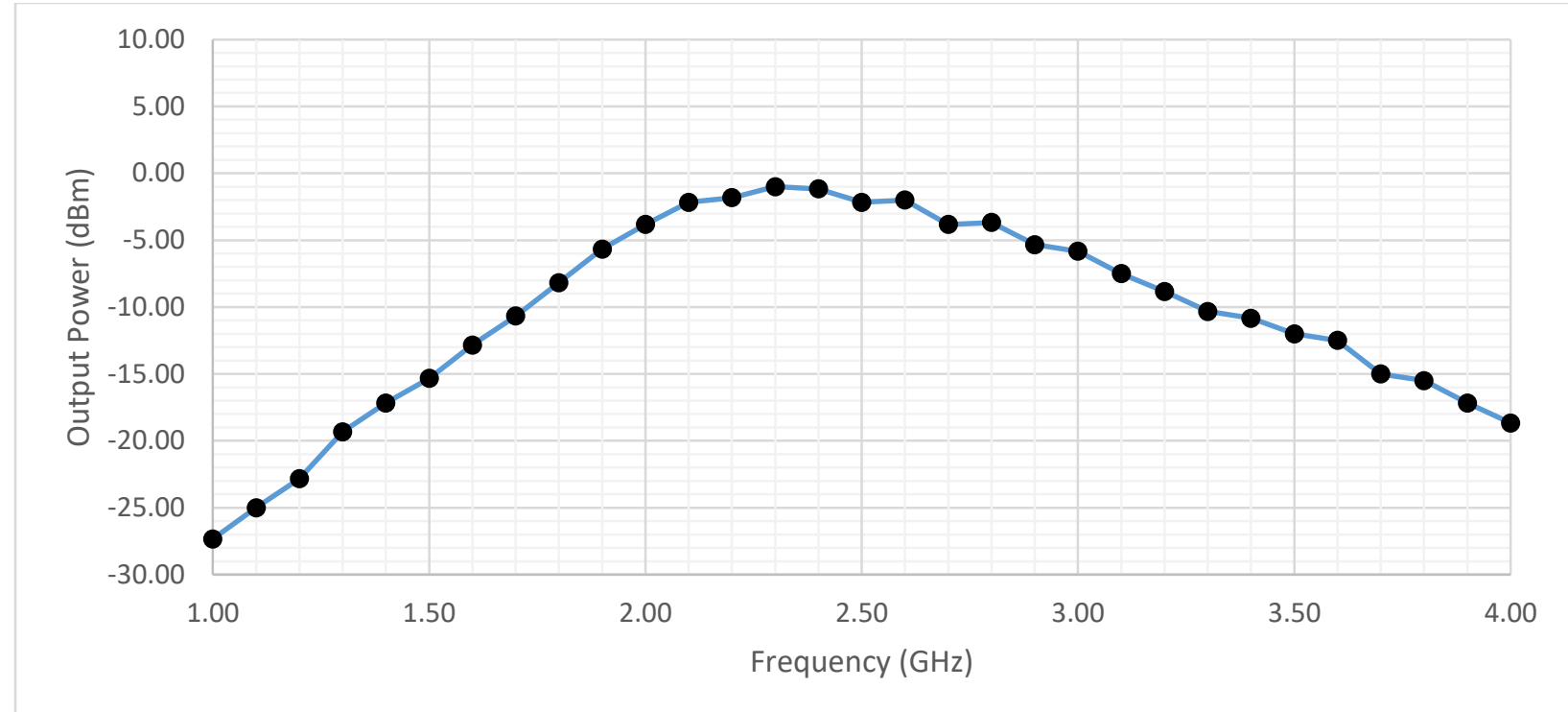

Figure 6.6: Measured output power $(\mathrm{dBm})$ over frequency $(\mathrm{GHz})$ at $\mathrm{P}_{\text {in }}=10 \mathrm{dBm}$.

The output power achieves a maximum of $-1.1 \mathrm{dBm}$ at the design frequency of $2.4 \mathrm{GHz}$ and is noticeably and dramatically reduced from the simulation results. The gain performance in the design bandwidth is negative indicated losses through the circuit with a magnitude in excess of $10 \mathrm{~dB}$.

The input frequency was fixed at $2.4 \mathrm{GHz}$ and the input power was swept from 0 to 14 $\mathrm{dBm}^{32}$. The output power at the fundamental frequency was measured and the results are shown in Figure 6.7 below.

\footnotetext{
${ }^{32}$ The maximum source power was $15 \mathrm{dBm}$ and the input harness losses were approximately $1 \mathrm{~dB}$.
} 


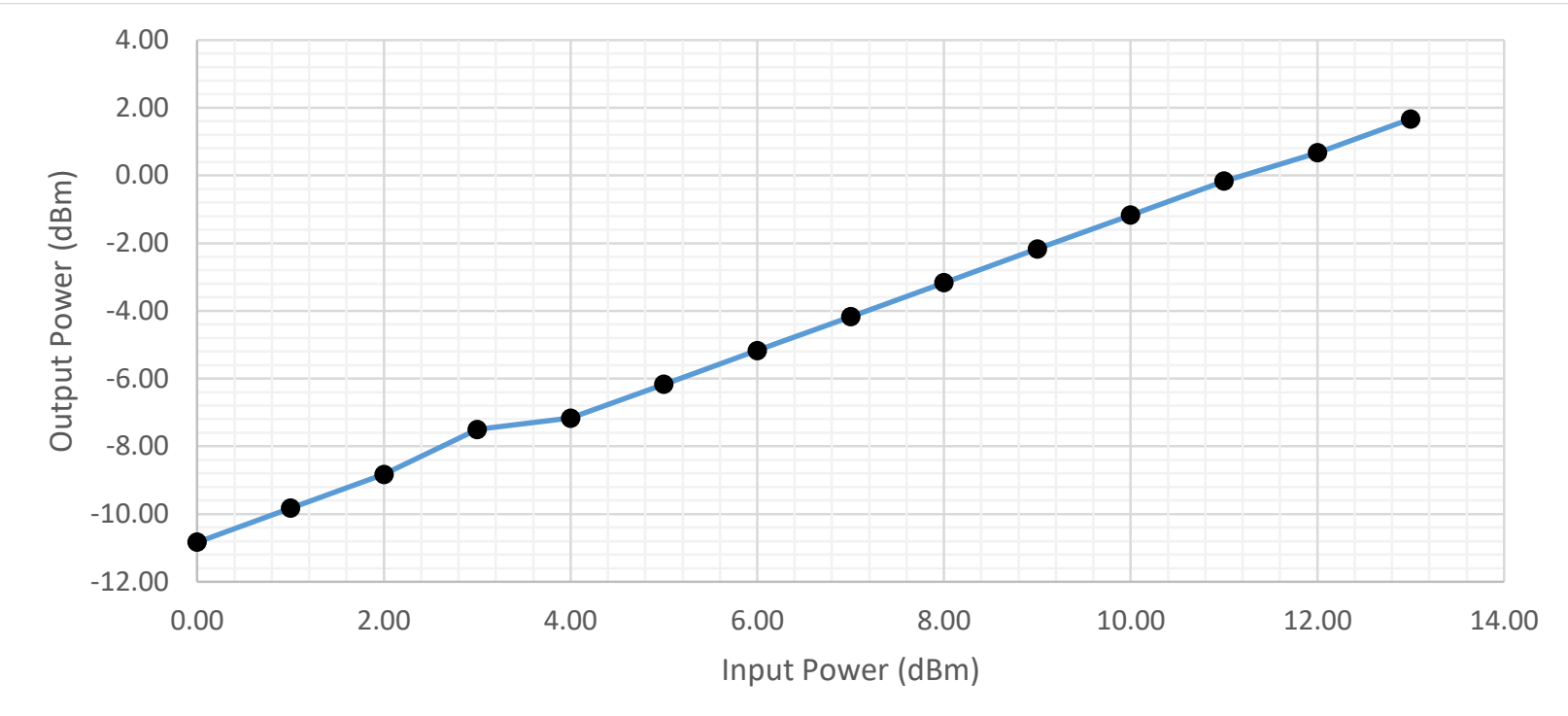

Figure 6.7: Measured output power $(\mathrm{dBm})$ over input power $(\mathrm{dBm})$ at $2.4 \mathrm{GHz}$.

The slope of the $\mathrm{P}_{\text {in }} \mathrm{V}$. $\mathrm{P}_{\text {out }}$ curve is linear throughout the sweep range and is consistent with the frequency performance observed in Figure 6.6. The strongly linear results indicate that the power amplifier - if it is indeed operating - is unlikely to be saturated.

\subsection{Analysis}

Although the operational frequency range of the circuit appears to be consistent with the design, the measured circuit's gain and output power performance is strongly negative. The following sub-sections will discuss various hypotheses for the performance discrepancy and what testing was completed to evaluate them. The formation and evaluation of these hypotheses happened in series (i.e. as one hypothesis was determined to be insufficient to explain the performance, another was formulated and tested). For clarity, they are presented in chronological order.

Before delving into these functional hypotheses, it is worth noting that a common culprit for poor circuit performance is a missed connection in the layout. Due to the complexity of this design, it was not possible to conduct a pin-to-pin LVS analysis. Instead, an equivalent 
representative schematic that connected the bias pad on the secondary of the input balun to the supply and rail pads on the primary of the output transformer was generated for LVS. This analysis did not check the primary side of the input balun or the secondary side of the output transformer; it is therefore possible that a missed connection in either of those two sub-circuits could lead to performance failure.

\subsubsection{Hypothesis 1: Input and Output Mismatches}

As observed at the beginning of section 6.2, after adjusting for harness losses, the circuit drew the expected current and the bias voltages were stable at the expected values. It was therefore assumed that the active devices were functional and consequently, the first hypothesis was that a mismatch in the passive output network was leading to a non-optimal loading condition. Although a mismatch at the input could also lead to poor performance, the small signal results indicated that at the design frequency the return loss was less than $-15 \mathrm{~dB}$ (Figure 6.5) and so this was considered unlikely. Focusing on the output matching network, a load pull was conducted to determine if adjusting the loading condition could recover the simulated performance.

\subsubsection{Load pull test bench}

The load pull was conducted at the Skyworks Solutions Inc. testing facility in Kanata, Ontario. The source and load impedances were varied using a set of mechanical stub tuners that adjust the source and load matches. The test bench block diagram is shown in Figure 6.8 below. 


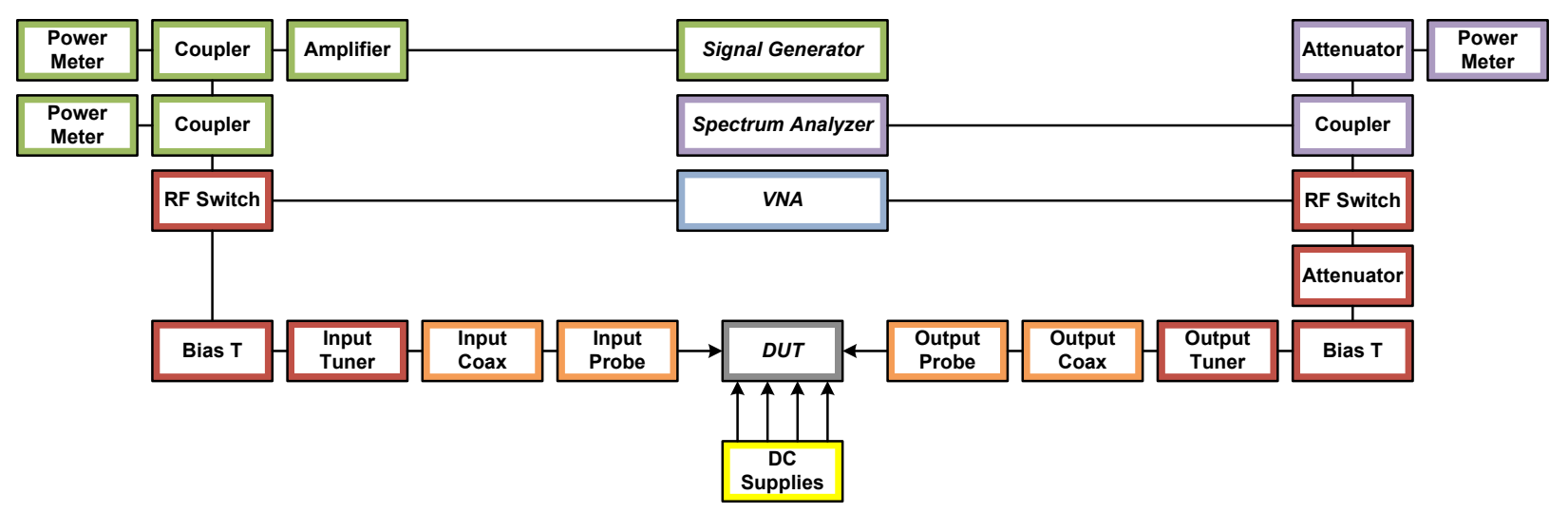

Figure 6.8: Block diagram for the load pull test bench.

The RF switches are used to select between small signal analysis using the VNA and large signal analysis using the signal generator and spectrum analyzer. The colour codes in the block diagram indicate the calibration group stages. A photograph of the test setup is shown in Figure 6.9 below.

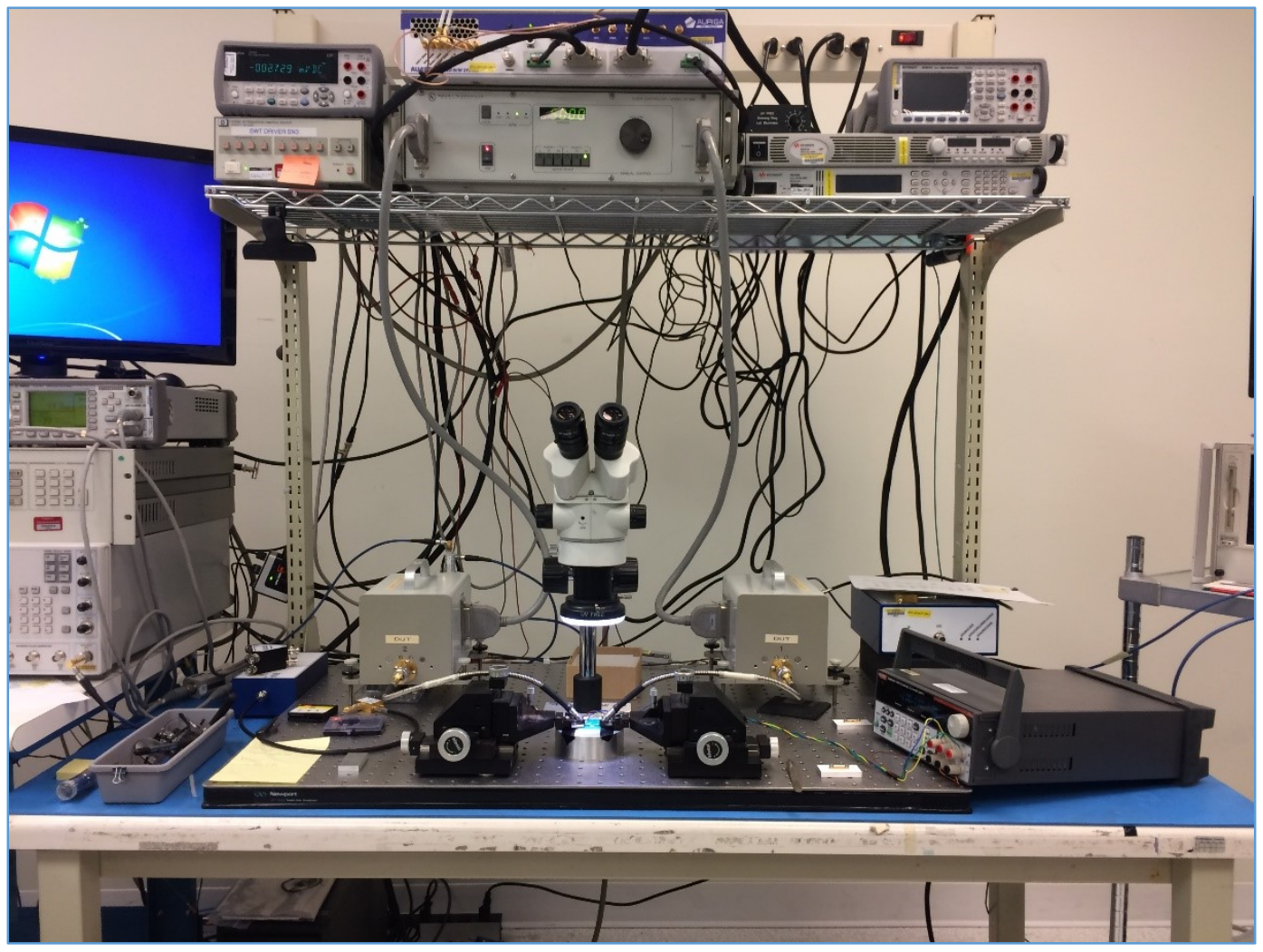

Figure 6.9: Photograph of the load pull test bench. 
The calibration plane was brought to the probe tips using the same calibration substrate used in the initial measurement phase. All other calibrations were completed in advance by Skyworks. Although the design frequency was $2.4 \mathrm{GHz}$, the calibration presets available from Skyworks were for $2.5 \mathrm{GHz}$. From Figure 6.5 , the return loss at $2.5 \mathrm{GHz}$ is reasonable at $10 \mathrm{~dB}$ however it was noted that this frequency fell outside of the designed locking range. In order to accommodate this, a settling time was introduced between each measurement to allow for pull-in. As there was no indication in which direction the mismatch was located, an evenly distributed set of test points were selected throughout the Smith chart.

\subsubsection{Measurement results}

The results of the load pull measurements are shown in Figure 6.10 below. The blue gain contours range from -24.5 to $-20 \mathrm{~dB}$ in $0.5 \mathrm{~dB}$ steps with the maximum gain of $-19.7 \mathrm{~dB}$ achieved at $(106.03+j 66.09) \Omega$. The maximum output power was $-9.70 \mathrm{dBm}$ and is collocated with the maximum gain point.

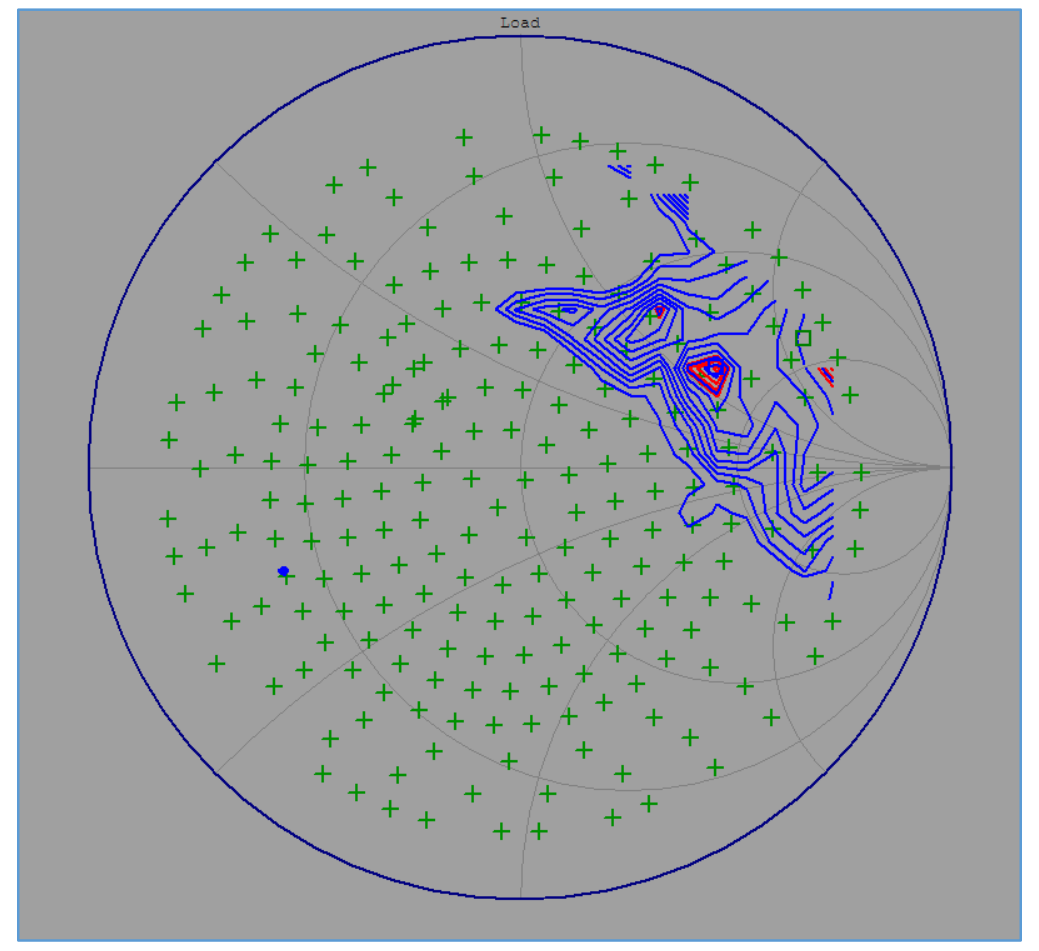


Figure 6.10: Smith chart showing constant gain (blue) and power (red) contour measurements

$$
\text { for } \mathrm{P}_{\mathrm{in}}=10 \mathrm{dBm} \text { at } 2.5 \mathrm{GHz} \text {. }
$$

\subsubsection{Analysis}

From Figure 6.10, the optimal loading condition for peak gain appears to be inductive, suggesting a tuning mismatch in the output transformer. The peak gain at that location, however, remains significantly reduced compared to the simulated performance. Not shown is the delivered input power which shows minimal variation from 8.20 to $8.60 \mathrm{dBm}$. This is consistent with the expected return loss both from simulation and small signal measurements and validates the assumption that mismatches are likely constrained to the output matching network.

In summary, the test indicated that there is possible output mismatch, which is expected to be contributing to the performance discrepancy. However, it was not believed to be sufficient to explain the observed behaviour on its own.

\subsubsection{Hypothesis 2: Oscillator Function and Stability}

After external adjustments to the output matching network did not recover the performance, problems with active device cores were considered. The unit power cells rely on functional oscillators in order to realize the gain enhancement observed in simulation. In the amplifier design, the oscillator transistors provide approximately $60 \%$ of the output current, corresponding to approximately $10 \mathrm{~dB}$ of gain. It was therefore hypothesized that since minimal gain was observed, one or more of the unit oscillators were not turning on. Due to time constraints, this hypothesis was tested solely in simulation by setting the oscillator gate bias voltage to zero.

\subsubsection{Simulation results}

To simulate the oscillators not turning on, the oscillator gate bias was set to $0 \mathrm{~V}$ and the small signal and large signal simulation tests were conducted. First, the input frequency was 
swept and the S-parameters extracted. The return loss $\left(\mathrm{S}_{11}\right)$ and forward gain $\left(\mathrm{S}_{21}\right)$ are plotted in Figure 6.11 below.

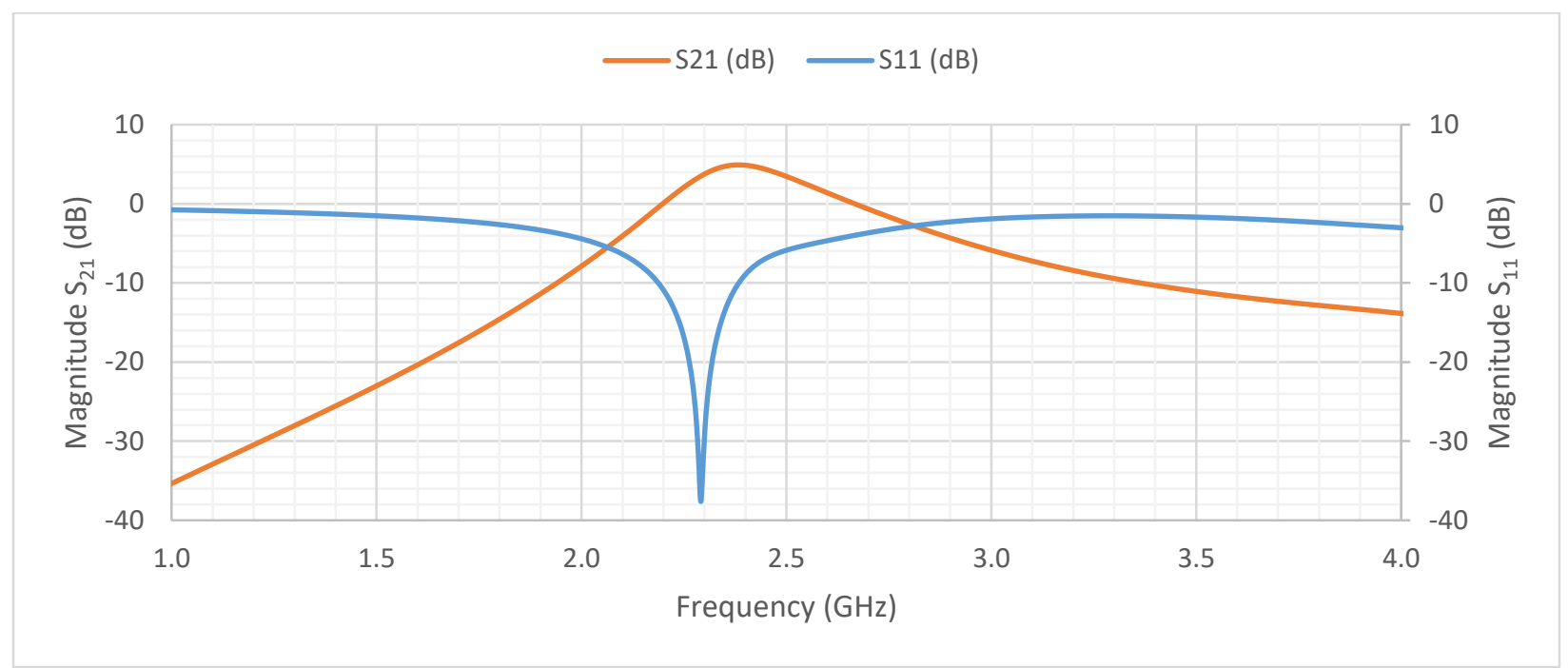

Figure 6.11: $\mathrm{S}_{11}$ (blue) and $\mathrm{S}_{21}$ (orange) measured over frequency with oscillators disabled.

The return loss curve has the notch shifted slightly lower in frequency and is missing the resonance feature that was observed in the simulation with the oscillators enabled (Figure 5.23 in section 5.6). The forward gain curve remains peaked at $2.4 \mathrm{GHz}$ but is reduced to $5 \mathrm{~dB}-$ an 13 $\mathrm{dB}$ drop from what was observed previously (Figure 5.23 in section 5.6). Next, the input power was swept and output power and gain extracted (Figure 6.12 below). 


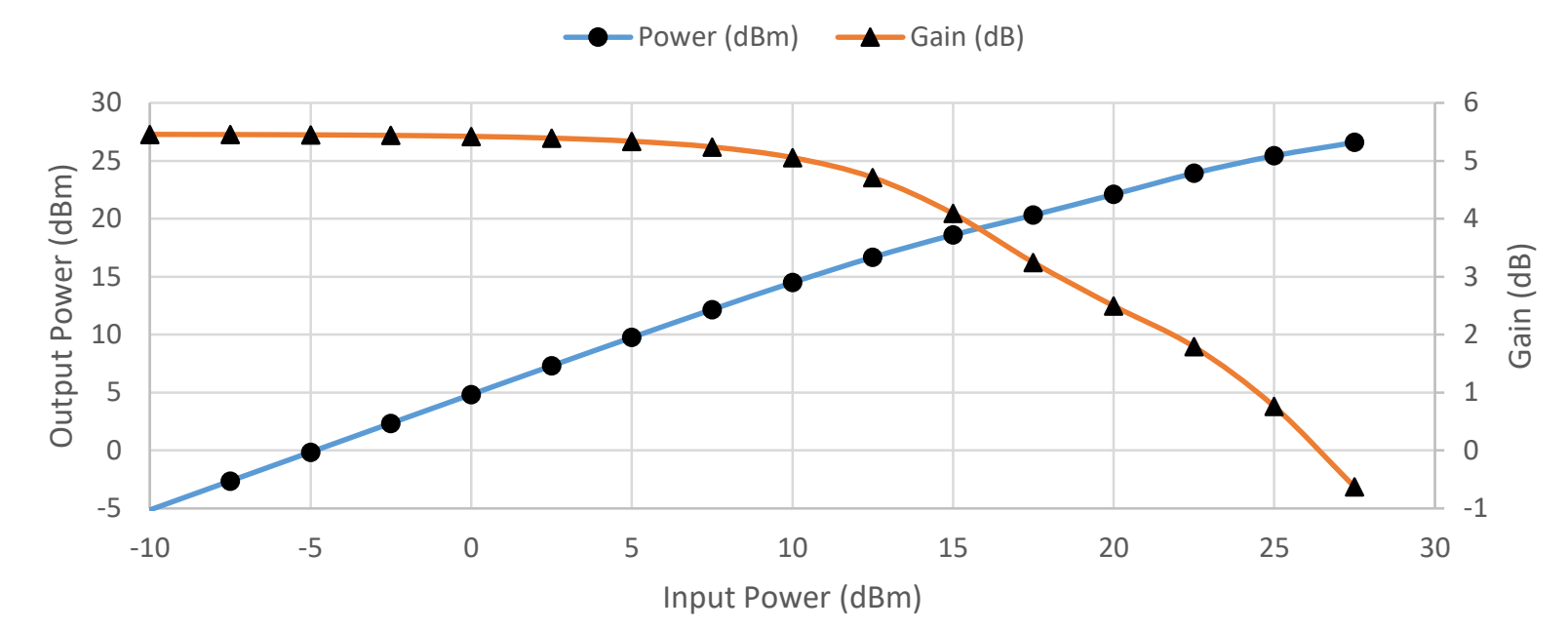

Figure 6.12: Output power (blue) and gain (orange) measured over input power with oscillators disabled.

The output power and gain curves show the same degradation observed in the small signal analysis. The gain is slightly greater than $5 \mathrm{~dB}$ in the linear region - down approximately $10 \mathrm{~dB}$ from the original results - and begins to roll off at $10 \mathrm{dBm}$ of input power.

\subsubsection{Analysis}

The performance degradation with the oscillators disabled is significant and could account for a portion of the observed degradation in measured performance. The cross-connection of the tank capacitors means that instabilities in any one of the oscillators will propagate to all of the oscillators in the ring. If one or more fail to oscillate (or lock), the adjacency assumption that permits the cross-connection would no longer hold. Under optimal circumstances, this would lead to no oscillations and the amplifier would 'default' to the normal performance of a single stage differential pair. A more severe (and likely) scenario is that the oscillators lead to additional instabilities that further degrade the performance. A test of the amplifier stability with the oscillators disabled was also conducted. The results are shown in Figure 6.13 below. 


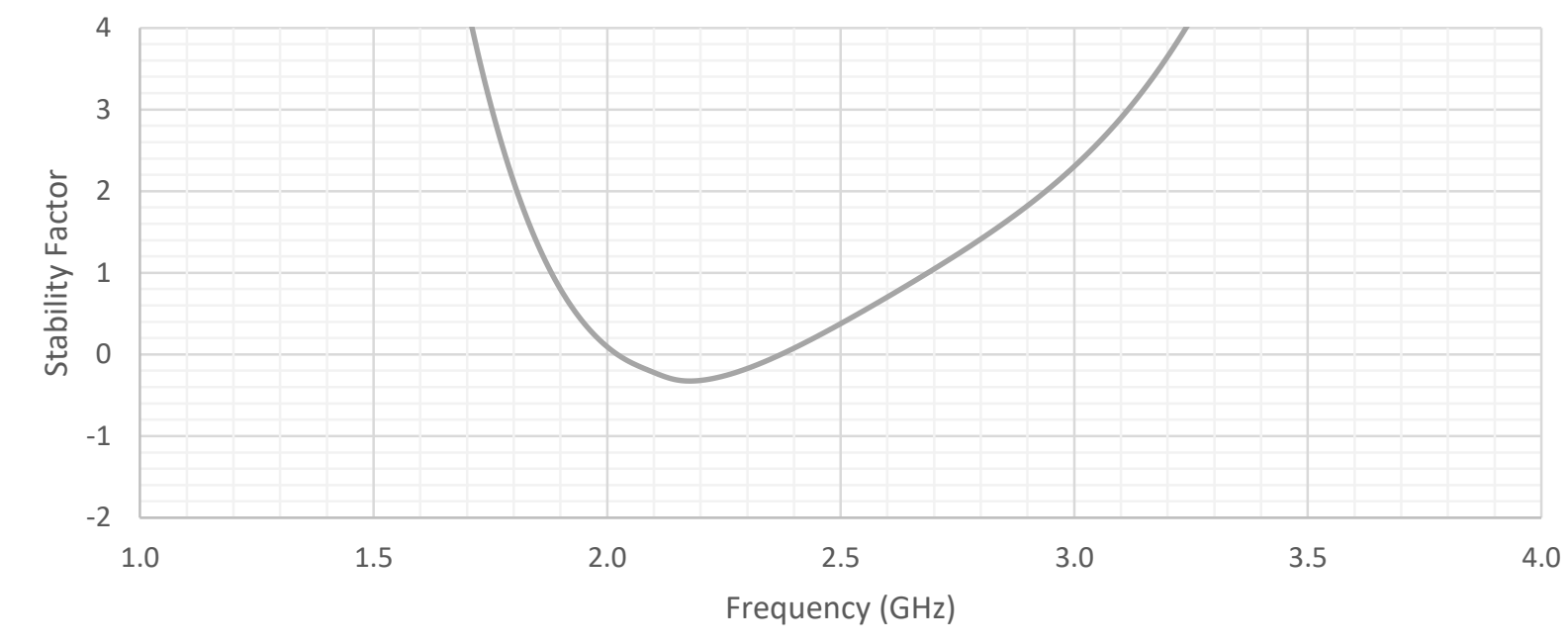

Figure 6.13: Stability factor measured over frequency with oscillators disabled.

Even with the oscillator disabled, the stability factor falls below unity throughout the desired band and therefore the circuit is not unconditionally stable. It is therefore possible that the parasitic reactances, not modeled in simulation, could lead to a loading condition that causes instability and degrades performance.

\subsubsection{Hypothesis 3: Other Sources of Loss}

A final consideration for the performance degradation is severe losses in the output network. This is in contrast to the first hypothesis in which a non-ideal output mismatch degraded the power cell performance (i.e. poor loading). If the output network efficiency is very low, the output power will be dramatically reduced since much of the available power will be dissipated as heat prior to reaching the load impedance. This hypothesis was not tested in simulation or by measurement due to time constraints. Instead, two potential root causes for output network losses are proposed.

\subsubsection{Leakage inductance and parasitic capacitance in output transformer}

The output network uses magnetic coupling to enable series combining of the unit power cell output voltages. This magnetic coupling can be modelled as a set of transformers, connected 
differentially on the primary side. From simulation, the coupling factor of the DAT was 0.6 implying large leakage inductances on the primary and secondary windings. From section 2.5.2.2, it was noted that tuning capacitances are required to ensure the L-network equivalents generated by the parasitic inductances and capacitances are tuned appropriately. The slab inductors themselves are expected to require significant tuning; however, this is conveniently supplied by the tank capacitor on the primary side. On the secondary side, the presence of a tuning capacitor did not appear to improve the performance and so was not included in the final implementation. It is possible that modelling of the output network geometry did not account for all parasitic reactances realized in fabrication and that this tuning capacitance was indeed necessary.

\subsubsection{Substrate loss due to poor isolation}

The other consideration is poor isolation of the magnetically coupled slabs leading to large currents flowing through the lossy substrate. There are two ways in which substrate currents are created by the slab inductors. The first is via capacitive coupling and the second is through magnetic coupling. In order to minimize the first, a patterned ground plane is typically introduced under large spiral inductors to prevent current from flowing into the lossy substrate. However, the ground plane capacitance will typically be higher than the substrate capacitance and will reduce the self-resonant frequency of the inductor [11:154]. To minimize the second, the substrate conductivity is reduced by blocking p-well implant underneath the inductor and limiting substrate current to the p-substrate.

The slab inductors were built by modifying the design kit transmission line component which does not include a metal ground plane but does include a high resistivity substrate mask. The high resistivity mask was retained, however, when simulating the full DAT in EMX, the simulator was configured such that this high resistivity was homogenous throughout the wafer as 
opposed to localized to the areas defined by the mask. Consequently, the substrate currents in the regions outside the mask would be under estimated. This may have artificially improved the simulated quality factor and created errors in the modelled inductance values. If these values were inaccurate with respect to the actual values realized in fabrication, the operation could vary from that predicted in simulation. Since the input network performance appears to align with the simulated results, and the load pull suggests that the ideal output match is significantly displaced from that predicted by simulation, this potential simulation error is considered a good candidate for future exploration. Unfortunately, this hypothesis was generated too late to be tested in time for the submission of this work.

\subsection{Chapter Summary}

In this chapter, the testing apparatus and measurement results were presented. The measured results did not align with those predicted by simulation and several hypotheses were proposed to explain the discrepancies. Although no conclusive indication as to the performance degradation was found, several paths forward were identified. These will be reviewed as part of the future work suggested in the next chapter. 


\section{Chapter 7: Conclusion}

This chapter begins by summarizing the thesis contributions and conclusions. It concludes with an overview of potential directions for future work including a discussion of possible breakout circuits and proposed locations for tunable components.

\subsection{Contributions and Conclusions}

The continued effort towards a fully integrated RF front end requires the exploration and evaluation of increasingly complex architectures. In this context, the novel integration of injection-locking for gain enhancement with the power enhancement of the DAT architecture is viewed as an important contribution towards the larger body of research in this field. The concept was successfully designed, implemented and fabricated in $0.13 \mu \mathrm{m} \mathrm{RF} \mathrm{CMOS} \mathrm{at} 2.4 \mathrm{GHz}$. The simulated performance indicates $\mathrm{P}_{1 \mathrm{~dB}}$ of $25.94 \mathrm{dBm}$ with a maximum gain of $14.5 \mathrm{~dB}, 22 \%$ PAE and locking range in excess of $500 \mathrm{MHz}$.

Although substantive discrepancies between the simulated and measured performance were observed, reasonable and testable hypotheses were generated and - where possible evaluated for consistency with the observed performance. Of particular importance is the observation that this circuit - more so than its constituent concept architectures - is particularly susceptible to parasitic capacitance at the output and may require additional high power tuning circuits for practical implementations. 


\subsection{Future Work}

As a proof of concept, this first iteration of the design, layout and fabrication cycle appears promising. Critical to the development of this concept will be the harmonization of simulation with results with measured results. On this basis, proposed future work is organized along the following three trajectories:

- first, the segmentation of the design into break out circuits with on-chip de-embedding structures to verify module functionality and performance;

- second, the introduction of tunable components in the unit power cells where circuit conditions permit safe operation; and,

- third, in the output network - where such safe conditions do not exist - the use of a higher fidelity simulation environment (e.g. HFSS) to more accurately analyze passive EM structures and expose potential parasitic reactances.

The third trajectory is fairly prescriptive; in essence, a recommendation to include additional rigor in the simulation tool chain. The first and second, however, require more fulsome treatment and are expanded in the following sub-sections.

\subsubsection{Proposed break out circuits}

In a circuit architecture of this complexity, it is useful to layout breakout circuits of key functional modules for isolated and independent analysis. These breakouts can be implemented on the same die, or alternatively, may be on a separate chip. Future work along this line would involve breaking out two of the functional modules from Figure 5.2, namely the integrated balun and unit power cell. 


\subsubsection{Breaking out the integrated balun}

The integrated balun serves the key function of on-chip single-end to differential signal conversion. The implementation is a center-tapped symmetrical transformer which - as discussed in section 2.5.2.2 - requires careful tuning to resonate out parasitic L-sections. The breakout would allow verification of its frequency and phase performance enabling tighter tuning and more accurate accounting of losses through the input network.

Additionally, the design and fabrication of several variants on a breakout chip prior to the final end-to-end implementation would allow the designer to select the optimal variant for the full circuit architecture.

\subsubsection{Breaking out the unit power cell}

The unit power cell is the central component of the design and independent validation of its function is key to identifying root causes for the observe simulation/measurement discrepancies. Breaking out the unit power cell poses two distinct problems:

1. how to generate the required high quality factor inductance to ac ground that is provided by the slab inductances in the end-to-end implementation?

2. how to couple the output to the test port in such a way that the appropriate load impedance is generated?

With respect to the former, the design inductance to ground is approximately $168 \mathrm{pH}$ (i.e. $1 / 2$ the total inductance of the slab) which is achievable with the kit inductors. The high quality factor may be realizable, nevertheless, the absence of a convenient low loss ac ground will require careful design of the dc bias network.

The latter problem of output signal coupling is particularly difficult as the unit design requires a differentially connected $12.5 \Omega$ load impedance. One possible solution is to use 
differential pads with an off-chip balun to couple the differential signal into the test equipment. Another solution is to design variant of the input network balun to present the appropriate impedance while simultaneously providing the differential to single-ended conversion. Finally, one could load the oscillator with a $12.5 \Omega$ differentially connected resistor and use buffers to couple the drain voltages/currents to output pads. In this final configuration, the power cell operation could be confirmed independent of the output power it generated.

\subsubsection{Proposed tunable components}

The injection-locked DAT requires careful tuning and is sensitive to fabrication variation. It is therefore proposed that on-chip tuning components (e.g. varactors) be included in future designs to permit post-fabrication optimization of the performance. There are two areas where onchip tuning is favourable: first, in the unit cell resonator and second, in the input matching network.

A schematic showing possible tuning component locations for the unit cell is shown in Figure 7.1 below.

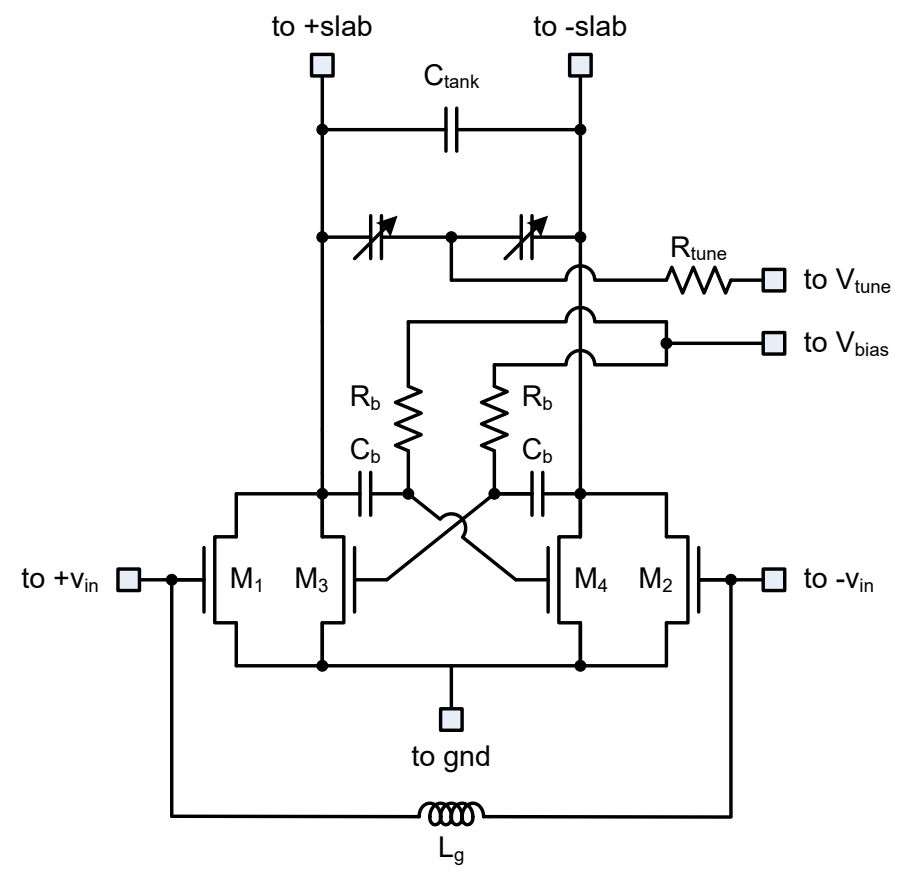

Figure 7.1: Unit power cell with possible location of tuning capacitors. 
The tuning varactors in the unit power cell would be used to compensate for variation in both the unit cell drain-source junction capacitance and the parasitic capacitance from the slab inductor.

The integrated balun is also anticipated to have some variation between the simulated and fabricated parasitic reactances; therefore, the input matching network would also benefit from tuning varactors connected in parallel with the existing tuning capacitances.

The output matching network experiences very large voltages and currents which makes on-chip tuning difficult as the varactor components typically suffer from the same breakdown voltage considerations of the active devices. Therefore, integrated output match tuning is not considered here. 


\section{List of References}

[1] International Telecommunications Union, "ITU-T Recommendation Y.2060: Overview of the Internet of things", 2012. [Online]. Available: http://www.itu.int/ITUT/recommendations/rec.aspx?rec=y.2060. [Accessed December 2016].

[2] T. Lee, "The Hardware Enablers for the Internet of Things - Part I", 13 January 2016. [Online]. Available: http://iot.ieee.org/newsletter/january-2015/the-hardware-enablers-forthe-internet-of-things-part-i.html. [Accessed December 2016].

[3] S. Jankowski et al., "The Internet of Things: Making sense of the next mega trend", 3 September 2014. [Online]. Available: http:// www.goldmansachs.com/ourthinking/outlook/internet-of-things/iot-report.pdf. [Accessed December 2016].

[4] T. Johansson and J. Fritzin, "A Review of Watt-Level CMOS RF Power Amplifiers", IEEE Trans. Microw. Theory Tech., vol. 62, no. 1, pp. 111-124, Jan. 2014.

[5] M. El-Desouki et al., "A Fully Integrated CMOS Power Amplifier Using Superharmonic Injection-Locking for Short-Range Applications”, IEEE Sensors J., vol. 11, no. 9, pp. 21492158, Sept. 2011.

[6] P. Reynaert and M. Steyaert, “A Fully Integrated CMOS RF Power Amplifier with Parallel Power Combining and Power Control", in 2005 IEEE Asian Solid-State Circuits Conference, Hsinchu, 2005, pp. 137-140.

[7] Boshi Jin et al., "Fully-integrated CMOS power amplifier design for WiMAX application with semi-lumped transformer," in 2008 6th IEEE International Conference on Industrial Informatics, Daejeon, 2008, pp. 181-185.

[8] M. D. Tsai et al., "A fully integrated multimode front-end module for GSM/EDGE/TDSCDMA/TD-LTE applications using a Class-F CMOS power amplifier," in 2017 IEEE 
International Solid-State Circuits Conference (ISSCC), San Francisco, CA, 2017, pp. 216217.

[9] H. Mosalam et al., "A high-efficiency good linearity 21 to $26.5 \mathrm{GHz}$ fully integrated power amplifier using $0.18 \mu \mathrm{m}$ CMOS technology," in 2016 IEEE 59th International Midwest Symposium on Circuits and Systems (MWSCAS), Abu Dhabi, 2016, pp. 1-4.

[10] J. W. M. Rogers, C. Plett and I . Marsland, Radio Frequency System Architecture and Design, Norwood, MA, United States: Artech House, 2013.

[11] J. W. M. Rogers and C. Plett, Radio Frequency Integrated Circuit Design, 2nd ed., Norwood, MA, United States: Artech House, 2010.

[12] I. Aoki, "Distributed Active Transformer for Integrated Power Amplification," Ph.D. dissertation, Caltech, Pasadena, CA, 2002.

[13] S.C. Cripps, RF Power Amplifiers for Wireless Communications, 2nd ed., Norwood, MA, United States: Artech House, 2006.

[14] I.J. Bahl, Fundamentals of RF and Microwave Transistor Amplifiers, Hoboken, NJ, United States: John Wiley \& Sons, Inc., 2009.

[15] A.A. Abidi, "RF CMOS comes of age", IEEE J. Solid-State Circuits, vol. 39, no. 4, pp. 549561, Apr. 2004.

[16] K.C. Tsai, "CMOS Power Amplifiers for Wireless Communication," Ph.D. dissertation, Univ. California, Berkley, 2007.

[17] S. Kee, "The Class E/F Family of Harmonic-Tuned Switching Power Amplifiers," Ph.D. dissertation, Caltech, Pasadenca, CA, 2002.

[18] J.R. Long, "Monolithic Transformers for Silicon RF IC Design”, IEEE J. Solid-State Circuits, vol. 35, no. 9, pp. 549-561, Sep. 2000. 
[19] C.H. Lin and H.Y. Chang, "A Broadband Injection-Locking Class-E Power Amplifier", IEEE Trans. Microw. Theory Tech., vol. 60, no. 10, pp. 3232-42, Oct. 2012.

[20] B. Razavi, "A Study of Injection Locking and Pulling in Oscillators", IEEE J. Solid-State Circuits, vol. 39, no. 9, pp. 1415-24, Sep. 2004. 